\title{
Search for dark matter and unparticles produced in association with a $Z$ boson in proton-proton collisions at $\sqrt{s}=8 \mathrm{TeV}$
}

\author{
V. Khachatryan et al. \\ (CMS Collaboration) \\ (Received 30 November 2015; published 22 March 2016)
}

\begin{abstract}
A search for evidence of particle dark matter (DM) and unparticle production at the LHC has been performed using events containing two charged leptons, consistent with the decay of a $Z$ boson, and large missing transverse momentum. This study is based on data collected with the CMS detector corresponding to an integrated luminosity of $19.7 \mathrm{fb}^{-1}$ of $p p$ collisions at the LHC at a center-of-mass energy of $8 \mathrm{TeV}$. No significant excess of events is observed above the number expected from the standard model contributions. The results are interpreted in terms of $90 \%$ confidence level limits on the DM-nucleon scattering cross section, as a function of the DM particle mass, for both spin-dependent and spinindependent scenarios. Limits are set on the effective cutoff scale $\Lambda$, and on the annihilation rate for DM particles, assuming that their branching fraction to quarks is $100 \%$. Additionally, the most stringent $95 \%$ confidence level limits to date on the unparticle model parameters are obtained.
\end{abstract}

DOI: 10.1103/PhysRevD.93.052011

\section{INTRODUCTION}

Ample evidence from astrophysical measurements supports the existence of dark matter (DM), which is assumed to be responsible for galactic gravitation that cannot be attributed to baryonic matter [1-3]. Recent DM searches have exploited a number of methods including direct detection [4-11], indirect detection [12,13], and particle production at colliders [14-26]. The currently favored possibility is that DM may take the form of weakly interacting massive particles. The study presented here considers a mechanism for producing such particles at the CERN LHC [27]. In this scenario, a $Z$ boson, produced in $\mathrm{pp}$ collisions, recoils against a pair of DM particles, $\chi \bar{\chi}$. The $Z$ boson subsequently decays into two charged leptons $\left(\ell^{+} \ell^{-}\right.$, where $\ell=e$ or $\mu$ ) producing a clean dilepton signature together with missing transverse momentum due to the undetected DM particles. In this analysis, the DM particle $\chi$ is assumed to be a Dirac fermion or a complex scalar particle of which the coupling to standard model (SM) quarks $q$ can be described by one of the effective interaction terms [28]:

$$
\begin{aligned}
& \text { Vector, spin independent (D5): } \frac{\bar{\chi} \gamma^{\mu} \chi \bar{q} \gamma_{\mu} q}{\Lambda^{2}} ; \\
& \text { Axial-vector, spin dependent (D8): } \frac{\bar{\chi} \gamma^{\mu} \gamma^{5} \chi \bar{q} \gamma_{\mu} \gamma^{5} q}{\Lambda^{2}} ; \\
& \text { Tensor, spin dependent (D9): } \frac{\bar{\chi} \sigma^{\mu \nu} \chi \bar{q} \sigma_{\mu \nu} q}{\Lambda^{2}} ; \\
& \text { Vector, spin independent (C3): } \frac{\chi^{\dagger} \stackrel{\leftrightarrow}{\partial_{\mu}} \chi \bar{q} \gamma^{\mu} q}{\Lambda^{2}}
\end{aligned}
$$

where $\Lambda$ parametrizes the effective cutoff scale for interactions between DM particles and quarks. The operators denoted by D5, D8, and D9 couple to Dirac fermions, while

*Full author list given at the end of the article.

Published by the American Physical Society under the terms of the Creative Commons Attribution 3.0 License. Further distribution of this work must maintain attribution to the author(s) and the published article's title, journal citation, and DOI.
C3 couples to complex scalars. The corresponding Feynman diagrams for production of a DM pair with a $Z$ boson and up to one jet are shown in Fig. 1. A search similar to the one presented here has been performed by the ATLAS Collaboration [26], where the DM particle is assumed to be a Dirac fermion and couples to either vector bosons or quarks.

The unparticle physics concept [29-32] is particularly interesting because it is based on scale invariance, which is anticipated in many beyond-the-SM physics scenarios [33-35]. The unparticle stuff of the scale-invariant sector 


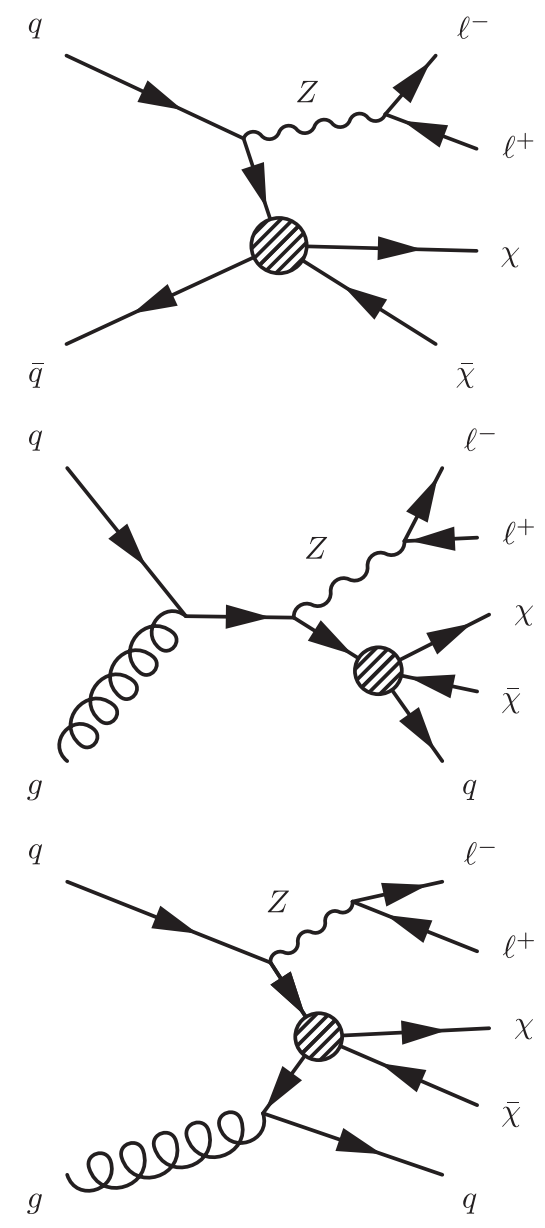

FIG. 1. The principal Feynman diagrams for the production of DM pairs in association with a $Z$ boson. In the middle and righthand diagrams, an additional quark is produced. The hatched circles indicate the interaction modeled with an effective field theory.

appears as a noninteger number of invisible massless particles. In this scenario, the SM is extended by introducing a scale-invariant Banks-Zaks $(\mathcal{B Z})$ field, which has a nontrivial infrared fixed point [36]. This field can interact with SM particles by exchanging heavy particles with a high mass scale $M_{\mathcal{U}}$. Below this mass scale, the coupling is nonrenormalizable, and the interaction is suppressed by powers of $M_{\mathcal{U}}$. The interaction Lagrangian density can be expressed as $\mathcal{L}_{\text {int }}=\mathcal{O}_{\mathrm{SM}} \mathcal{O}_{\mathcal{B Z}} / M_{\mathcal{U}}^{k}$, where $\mathcal{O}_{\mathrm{SM}}$ is the operator for the SM field with scaling dimension $d_{\mathrm{SM}}$, $\mathcal{O}_{\mathcal{B Z}}$ is the operator for the $\mathcal{B Z}$ field with scaling dimension $d_{\mathcal{B Z}}$, and $k=d_{\mathrm{SM}}+d_{\mathcal{B Z}}-4>0$. At an energy scale of $\Lambda_{\mathcal{U}}$, dimensional transmutation is induced by renormalization effects in the scale-invariant $\mathcal{B Z}$ sector, and $\mathcal{O}_{\mathcal{B Z}}$ can be matched to a new set of operators below $\Lambda_{\mathcal{U}}$ with the interaction form

$$
\mathcal{L}_{\text {int }}^{\text {eff }}=C_{\mathcal{U}} \frac{\Lambda_{\mathcal{U}}^{d_{\mathcal{Z} Z}-d_{\mathcal{U}}}}{M_{\mathcal{U}}^{k}} \mathcal{O}_{\mathrm{SM}} \mathcal{O}_{\mathcal{U}}=\frac{\lambda}{\Lambda_{\mathcal{U}}^{d_{\mathcal{U}}}} \mathcal{O}_{\mathrm{SM}} \mathcal{O}_{\mathcal{U}}
$$

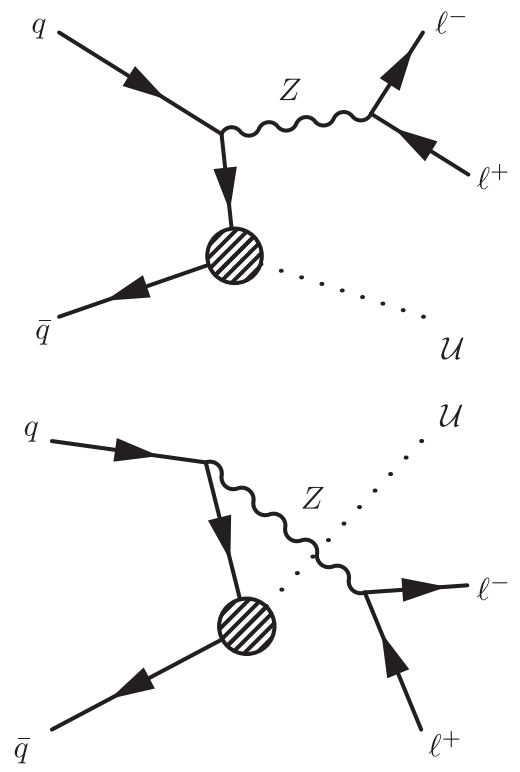

FIG. 2. Feynman diagrams for unparticle production in association with a $Z$ boson. The hatched circles indicate the interaction modeled with an effective field theory.

in which $C_{\mathcal{U}}$ is a normalization factor fixed by the matching, $d_{\mathcal{U}}$ represents the possible noninteger scaling dimension of the unparticle operator $\mathcal{O}_{\mathcal{U}}$, and the parameter $\lambda=C_{\mathcal{U}} \Lambda_{\mathcal{U}}^{d_{\mathcal{Z}}} / M_{\mathcal{U}}^{k}$ is a measure of the coupling between SM particles and unparticles. In general, an unparticle does not have a fixed invariant mass but has instead a continuous mass spectrum, and its real production in low energy processes described by the effective field theory in Eq. (1) can give rise to an excess of missing energy because of the possible nonintegral values of the scaling dimension $d_{\mathcal{U}}$. In the past, the reinterpretation [37] of LEP single-photon data has been used to set unparticle limits. A recent search for unparticles at CMS [14] in monojet final states has shown no evidence for their existence. In this paper, a scalar unparticle with real emission is considered, and the scaling dimension $d_{\mathcal{U}}>1$ is constrained by the unitarity condition. Figure 2 shows the two tree-level diagrams considered in this paper for the production of unparticles associated with a $Z$ boson.

Both the DM and unparticle scenarios considered in this analysis produce a dilepton $\left(e^{+} e^{-}\right.$or $\left.\mu^{+} \mu^{-}\right)$signature consistent with a $Z$ boson, together with a large magnitude of missing transverse momentum. The analysis is based on the full data set recorded by the CMS detector in 2012, which corresponds to an integrated luminosity of $19.7 \pm$ $0.5 \mathrm{fb}^{-1}$ [38] at a center-of-mass energy of $8 \mathrm{TeV}$.

\section{CMS DETECTOR}

The CMS detector is a multipurpose apparatus well suited to study high transverse momentum $\left(p_{\mathrm{T}}\right)$ physics processes in pp collisions. The central feature of the CMS 
apparatus is a superconducting solenoid of $6 \mathrm{~m}$ internal diameter, providing a magnetic field of $3.8 \mathrm{~T}$. Within the superconducting solenoid volume are a silicon pixel and strip tracker, a lead tungstate crystal electromagnetic calorimeter (ECAL), and a brass and scintillator hadron calorimeter (HCAL), each composed of a barrel and two end cap sections. Forward calorimeters extend the pseudorapidity [39] coverage provided by the barrel and end cap detectors. The electromagnetic calorimeter consists of 75 848 lead tungstate crystals, which provide coverage in pseudorapidity $|\eta|<1.479$ in a barrel region and $1.48<$ $|\eta|<3.00$ in two end cap regions (EE). A preshower detector consisting of two planes of silicon sensors interleaved with a total of $3 X_{0}$ of lead is located in front of the EE. The electron momentum is estimated by combining the energy measurement in the ECAL with the momentum measurement in the tracker. The momentum resolution for electrons with $p_{\mathrm{T}} \approx 45 \mathrm{GeV}$ from $Z \rightarrow e e$ decays ranges from $1.7 \%$ for nonshowering electrons in the barrel region to $4.5 \%$ for showering electrons in the end caps [40]. Muons are measured in the pseudorapidity range $|\eta|<2.4$, with gas-ionization detectors embedded in the steel fluxreturn yoke outside the solenoid. The muon detection planes are made using three technologies: drift tubes, cathode strip chambers, and resistive plate chambers. Matching muons to tracks measured in the silicon tracker results in a relative transverse momentum resolution for muons with $20<p_{\mathrm{T}}<100 \mathrm{GeV}$ of $1.3-2.0 \%$ in the barrel and better than $6 \%$ in the end caps. The $p_{\mathrm{T}}$ resolution in the barrel is better than $10 \%$ for muons with $p_{\mathrm{T}}$ up to $1 \mathrm{TeV}$ [41]. The first level of the CMS trigger system, composed of custom hardware processors, uses information from the calorimeters and muon detectors to select the most interesting events, in a fixed time interval of less than $4 \mu \mathrm{s}$. The high-level trigger processor farm further decreases the event rate from around 100 to less than $1 \mathrm{kHz}$, before data storage. A more detailed description of the CMS detector, together with a definition of the coordinate system used and the relevant kinematic variables, can be found in Ref. [39]. Variables of particular relevance to the present analysis are the missing transverse momentum vector $\vec{p}_{\mathrm{T}}^{\text {miss }}$ and the magnitude of this quantity, $E_{\mathrm{T}}^{\text {miss }}$. The quantity $\vec{p}_{\mathrm{T}}^{\text {miss }}$ is defined as the projection on the plane perpendicular to the beams of the negative vector sum of the momenta of all reconstructed particles in an event.

\section{SIMULATION}

Samples of simulated DM particle events are generated using MADGRAPH 5.2.1 [42] matched to PYTHIA 6.4.26 [43] using tune $\mathrm{Z} 2 *$ for parton showering and hadronization. The PYTHIA $6 \mathrm{Z2} *$ tune uses the CTEQ6L [44] parton distribution set. This tune is derived from the $\mathrm{Z} 1$ tune [45], which is based on CTEQ5L. The effective cutoff scale $\Lambda$ is set to $1 \mathrm{TeV}$. The events for the unparticle models are generated with PYTHIA 8.1 [46-48] assuming a
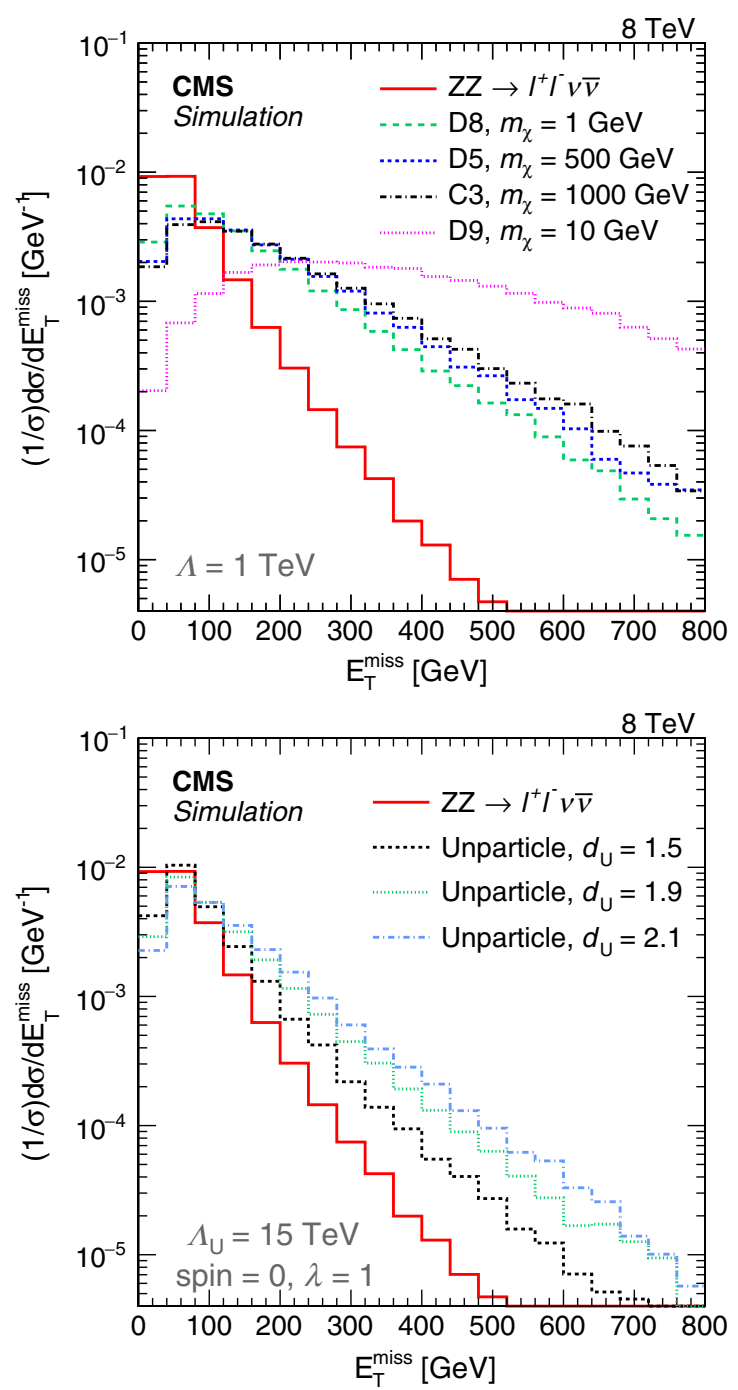

FIG. 3. The distribution in $E_{\mathrm{T}}^{\mathrm{miss}}$ at the generator level, for DM (left) and unparticle (right) scenarios. The DM curves are shown for different $m_{\chi}$ with vector (D5), axial-vector (D8), and tensor (D9) coupling for Dirac fermions and vector (C3) coupling for complex scalar particles. The unparticle curves have the scalar unparticle coupling $\lambda$ between unparticle and SM fields set to 1 , with the scaling dimension $d_{\mathcal{U}}$ ranging from 1.5 to 2.1. The SM background $Z Z \rightarrow \ell^{-} \ell^{+} \nu \bar{\nu}$ is shown as a red solid curve.

renormalization scale $\Lambda_{\mathcal{U}}=15 \mathrm{TeV}$, using tune 4C [49] for parton showering and hadronization. We evaluate other values of $\Lambda_{\mathcal{U}}$ by rescaling the cross sections as needed. The parameter $\Lambda_{\mathcal{U}}$ acts solely as a scaling factor for the cross section and does not influence the kinematic distributions of unparticle production [48]. Figure 3 shows the distribution of $E_{\mathrm{T}}^{\text {miss }}$ at the generator level for both $\mathrm{DM}$ and unparticle production. In the unparticle scenario, the events with larger scaling dimension $d_{\mathcal{U}}$ tend to have a broader $E_{\mathrm{T}}^{\text {miss }}$ distribution. For DM production, the shape of the $E_{\mathrm{T}}^{\text {miss }}$ is similar for couplings D5, D8, and C3, where the vector or axial vector couplings tend to produce nearly 
back-to-back DM particles. This configuration is less strongly favored for the tensor couplings, and thus the D9 couplings show a much broader $E_{\mathrm{T}}^{\text {miss }}$ distribution.

The POWHEG 2.0 [50-54] event generator is used to produce samples of events for the $t \bar{t}$ and $t W$ background processes. The $Z Z, W Z$, and Drell-Yan (DY, $\left.Z / \gamma^{*} \rightarrow \ell^{+} \ell^{-}\right)$processes are generated using the MADGRAPH 5.1.3 [55] event generator. The default set of parton distribution functions (PDFs) CTEQ6L [56] is used for generators that are leading order (LO) in $\alpha_{s}$, while the CT10 [57] set is used for next-to-leading-order (NLO) generators. The NLO calculations are used for background cross sections, whereas only LO calculations are available for the signal processes. For all Monte Carlo (MC) samples, the detector response is simulated using a detailed description of the CMS detector, based on the GEANT4 package [58]. Minimum bias events are superimposed on the simulated events to emulate the additional $\mathrm{pp}$ interactions per bunch crossing (pileup). All MC samples are corrected to reproduce the pileup distribution as measured in the data. The average number of pileup events per proton bunch crossing is about 20 for the 2012 data sample.

\section{EVENT RECONSTRUCTION}

Events are collected by requiring dilepton (ee or $\mu \mu)$ triggers with thresholds of $p_{\mathrm{T}}>17$ and $8 \mathrm{GeV}$ for the leading and subleading leptons, respectively. Single-lepton triggers with thresholds of $p_{\mathrm{T}}>27(24) \mathrm{GeV}$ for electrons (muons) are also included to recover residual trigger inefficiencies. Prior to the selection of leptons, a primary vertex must be selected as the event vertex. The vertex with largest value of $\sum p_{T}^{2}$ for the associated tracks is selected. Simulation studies show that this requirement correctly selects the event vertex in more than 99\% of both signal and background events. The lepton candidate tracks are required to be compatible with the event vertex.

A particle-flow (PF) event algorithm $[59,60]$ reconstructs and identifies each individual particle with an optimized combination of information from the various elements of the CMS detector. The energy of photons is directly obtained from the ECAL measurement, corrected for zero-suppression effects. The energy of electrons is determined from a combination of the electron momentum at the event vertex as determined by the tracker, the energy of the corresponding ECAL cluster, and the energy sum of all bremsstrahlung photons spatially compatible with originating from the electron track. The energy of muons is obtained from the curvature of the corresponding track. The energy of charged hadrons is determined from a combination of its momentum measured in the tracker and the matching ECAL and HCAL energy deposits, corrected for zero-suppression effects and for the response function of the calorimeters to hadronic showers. Finally, the energy of neutral hadrons is obtained from the corresponding corrected ECAL and HCAL energy.
Electron candidates are reconstructed using two algorithms [40]: in the first, energy clusters in the ECAL are matched to signals in the silicon tracker, and in the second, tracks in the silicon tracker are matched to ECAL clusters. The electron candidates used in the analysis are required to be reconstructed by both algorithms. To reduce the electron misidentification rate, the candidates have to satisfy additional identification criteria that are based on the shape of the electromagnetic shower in the ECAL. In addition, the electron track is required to originate from the event vertex and to match the shower cluster in the ECAL. Electron candidates with an ECAL cluster in the transition region between the ECAL barrel and end cap $(1.44<|\eta|<1.57)$ are rejected because the reconstruction of an electron object in this region is not optimal. Candidates that are identified as coming from photon conversions [40] in the detector material are explicitly removed.

Muon candidate reconstruction is also based on two algorithms: in the first, tracks in the silicon tracker are matched with at least one muon segment in any detector plane of the muon system, and in the second algorithm, a combined fit is performed to hits in both the silicon tracker and the muon system [41]. The muon candidates in this analysis are required to be reconstructed by both algorithms and to be further identified as muons by the PF algorithm. To reduce the muon misidentification rate, additional identification criteria are applied based on the number of space points measured in the tracker and in the muon system, the fit quality of the muon track, and its consistency with the event vertex location.

Leptons produced in the decay of $Z$ bosons are expected to be isolated from hadronic activity in the event. Therefore, an isolation requirement is applied based on the sum of the momenta of the PF candidates found in a cone of radius $R=\sqrt{(\Delta \eta)^{2}+(\Delta \phi)^{2}}=0.4$ around each lepton, where $\phi$ is the azimuthal angle. The isolation sum is required to be smaller than $15 \%(20 \%)$ of the $p_{\mathrm{T}}$ of the electron (muon). To correct for the contribution to the isolation sum from pileup interactions and the underlying event, a median energy density $(\rho)$ is determined on an event-by-event basis using the method described in Ref. [61]. For each electron, the mean energy deposit in the isolation cone of the electron, coming from other $\mathrm{pp}$ collisions in the same bunch crossing, is estimated following the method described in Ref. [40] and subtracted from the isolation sum. For muon candidates, only charged tracks associated with the event vertex are included. The sum of the $p_{\mathrm{T}}$ for charged particles not associated with the event vertex in the cone of interest is rescaled by a factor corresponding to the average neutral to charge energy densities in jets and subtracted from the isolation sum.

Jets are reconstructed from PF candidates by using the anti- $k_{\mathrm{T}}$ clustering algorithm [62] with a distance parameter of 0.5 , as implemented in the FASTJET package $[63,64]$. Jets are found over the full calorimeter acceptance, $|\eta|<5$. The 
jet momentum is defined as the vector sum of all particle momenta assigned to the jet and is found in the simulation to be within $5 \%$ to $10 \%$ of the true hadron-level momentum over the whole $p_{\mathrm{T}}$ range and detector acceptance. An overall energy subtraction is applied to correct for the extra energy clustered in jets due to pileup, following the procedure described in Ref. [65]. In the subtraction, the charged particle candidates associated with secondary vertices reconstructed in the event are also included. Other jet energy scale corrections applied are derived from simulation and are confirmed by measurements of the energy balance in dijet and $\gamma+$ jets events.

\section{EVENT SELECTION}

An initial preselection with a large yield is used to validate the background model and is followed by a final selection that is designed to give maximal sensitivity to the signal. Selected events are required to have exactly two well-identified, isolated leptons with the same flavor and opposite charge $\left(e^{+} e^{-}\right.$or $\left.\mu^{+} \mu^{-}\right)$, each with $p_{\mathrm{T}}>20 \mathrm{GeV}$. The invariant mass of the lepton pair is required to be within $\pm 10 \mathrm{GeV}$ of the nominal mass of the $Z$ boson. Only leptons within the pseudorapidity range of $|\eta|<2.4(2.5)$ for muons (electrons) are considered. To reduce the background from the $W Z$ process where the $W$ boson decays leptonically, events are removed if an additional electron or muon is reconstructed with $p_{\mathrm{T}}>10 \mathrm{GeV}$. As a very loose preselection requirement, the dilepton transverse momentum $\left(p_{\mathrm{T}}^{\ell \ell}\right)$ is required to be larger than $50 \mathrm{GeV}$ to reject the bulk of DY background events.

Since only a small amount of hadronic activity is expected in the final state of both DM and unparticle events, any event having two or more jets with $p_{\mathrm{T}}>$ $30 \mathrm{GeV}$ is rejected. Top quark decays, which always involve the emission of $b$ quarks, are further suppressed with the use of techniques based on soft-muon and $b$-jet tagging. The rejection of events with soft muons having $p_{\mathrm{T}}>3 \mathrm{GeV}$ reduces the background from semileptonic $b$ decays. The $b$-jet tagging technique employed is based on the "combined secondary vertex" algorithm $[66,67]$. This algorithm selects a group of tracks forming a secondary vertex within a jet and generates a likelihood discriminant to distinguish between $b$ jets and jets originating from light quarks, gluons, or charm quarks. The applied threshold provides, on average, $80 \%$ efficiency for tagging jets originating from $b$ quarks and $10 \%$ probability of lightflavor jet misidentification. The $b$-tagged jet is required to have $p_{\mathrm{T}}>20 \mathrm{GeV}$ and to be reconstructed within the tracker acceptance volume $(|\eta|<2.5)$.

The final selection is optimized for DM and unparticle signals to obtain the best expected cross section limit at $95 \% \mathrm{CL}$ using four variables, $E_{\mathrm{T}}^{\text {miss }}, \Delta \phi_{\ell \ell, \vec{p}_{\mathrm{T}}^{\text {miss }}}$, $\left|E_{\mathrm{T}}^{\text {miss }}-p_{\mathrm{T}}^{\ell \ell}\right| / p_{\mathrm{T}}^{\ell \ell}$, and $u_{\|} / p_{\mathrm{T}}^{\ell \ell}$, where $u_{\|}$is defined as the component of $\vec{u}=-\vec{p}_{\mathrm{T}}^{\text {miss }}-\vec{p}_{\mathrm{T}}{ }^{\ell \ell}$ parallel to the
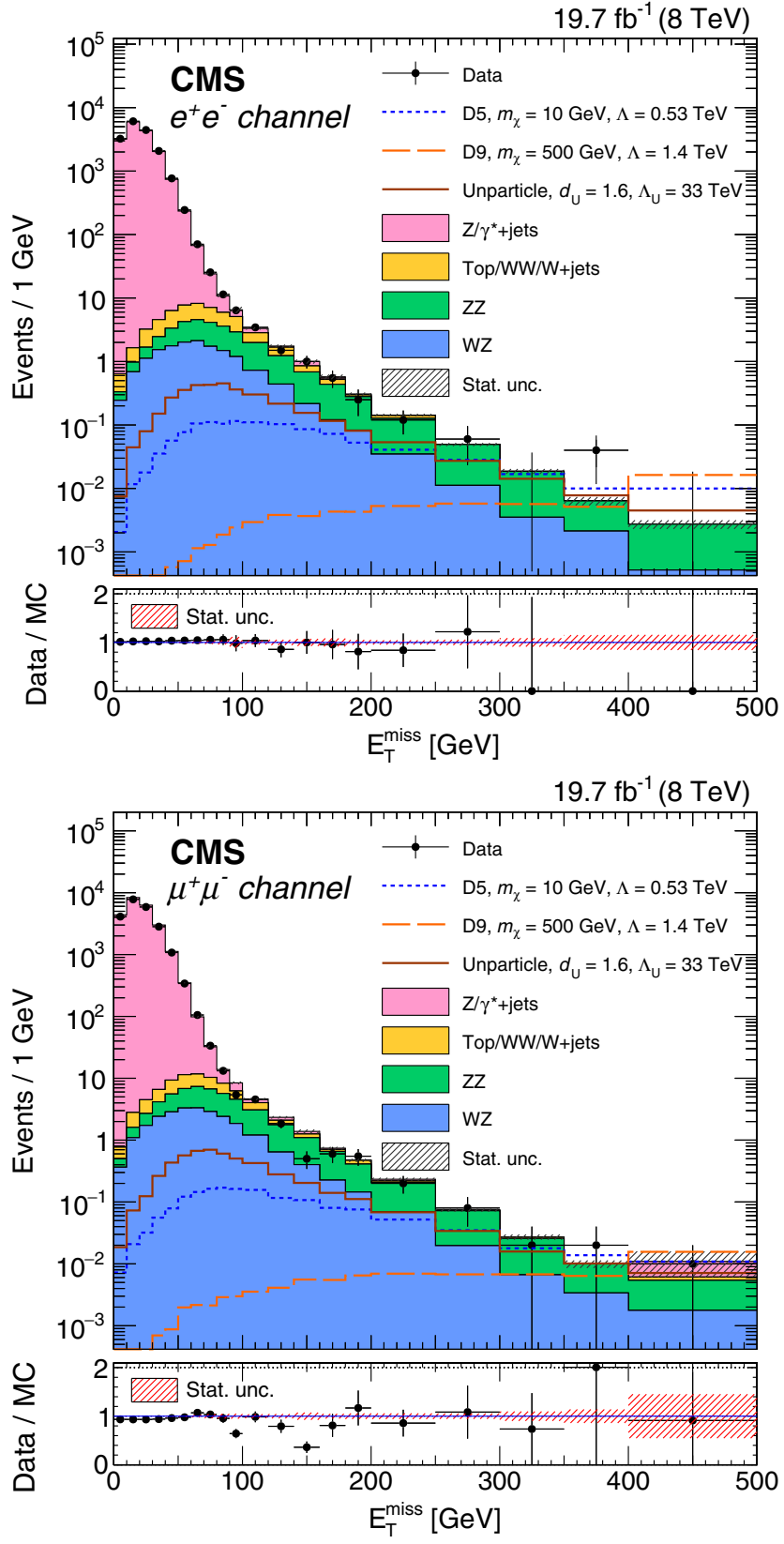

FIG. 4. The distribution of $E_{\mathrm{T}}^{\text {miss }}$ after preselection for the $Z \rightarrow e^{+} e^{-}$(left) and $Z \rightarrow \mu^{+} \mu^{-}$(right) channels. Expected signal distributions are shown for Dirac fermions with vector or tensor couplings and for unparticles. The total statistical uncertainty in the overall background is shown as a hatched region. The horizontal bars on the data points indicate the bin width. Overflow events are included in the rightmost bins.

direction of $\vec{p}_{\mathrm{T}} \ell \ell$. The last three variables effectively suppress background processes such as DY and topquark production. If the best expected significance is used in the optimization, instead of the best expected limit, very similar results are obtained. In both electron and muon channels, a mass-independent event selection followed by a fit to the shape of the transverse mass 
TABLE I. Summary of selections used in the analysis.

\begin{tabular}{lcc}
\hline \hline & Variable & Requirements \\
\hline Preselection & $p_{\mathrm{T}}^{\ell}$ & $>20 \mathrm{GeV}$ \\
& $\left|m_{\ell \ell}-m_{Z}\right|$ & $<10 \mathrm{GeV}$ \\
& Jet counting & $\leq 1$ jets with $p_{\mathrm{T}}^{\mathrm{j}}>30 \mathrm{GeV}$ \\
& $p_{\mathrm{T}}^{\ell \ell}$ & $>50 \mathrm{GeV}$ \\
& 3rd-lepton veto & $p_{\mathrm{T}}^{\ell}>10 \mathrm{GeV}$ \\
Selection & Top quark veto & Veto on $b$ jets and soft muon \\
& $\left|u_{\|} / p_{\mathrm{T}}^{\ell \ell}\right|$ & $<1$ \\
& $E_{\mathrm{T}}^{\text {miss }}$ & $>80 \mathrm{GeV}$ \\
& $\Delta \phi_{\ell \ell, \vec{p}_{\mathrm{T}}^{\text {miss }}}$ & $>2.7 \mathrm{rad}$ \\
& $\left|E_{\mathrm{T}}^{\mathrm{miss}}-p_{\mathrm{T}}^{\ell \ell}\right| / p_{\mathrm{T}}^{\ell \ell}$ & $<0.2$ \\
\hline \hline
\end{tabular}

$m_{\mathrm{T}}=\sqrt{2 p_{\mathrm{T}}^{\ell \ell} E_{\mathrm{T}}^{\text {miss }}\left(1-\cos \Delta \phi_{\ell \ell, \vec{p}_{\mathrm{T}}^{\text {miss }}}\right)}$ distribution is used to discriminate between the signal and the backgrounds. For each set of selection requirements considered, the full analysis, including the estimation of backgrounds and the systematic uncertainties, is repeated. The final selection criteria obtained after optimization for both the electron and muon channels are $E_{\mathrm{T}}^{\text {miss }}>80 \mathrm{GeV}$, $\Delta \phi_{\ell \ell, \vec{p}_{\mathrm{T}}^{\text {miss }}}>2.7,\left|u_{\|} / p_{\mathrm{T}}^{\ell \ell}\right|<1$, and $\left|E_{\mathrm{T}}^{\text {miss }}-p_{\mathrm{T}}^{\ell \ell}\right| / p_{\mathrm{T}}^{\ell \ell}<0.2$. This common selection is applied to both the DM and unparticle searches because the optimization results are very similar for both signals. A summary of the preselection and final selection criteria for the final analysis is listed in Table I. Figure 4 shows the distributions of $E_{\mathrm{T}}^{\text {miss }}$ after preselection, in the $e e$ and $\mu \mu$ channels. Good agreement is found between the observed distributions and the background prediction, which is described in the following section.

\section{BACKGROUND ESTIMATION}

The $Z Z$ and $W Z$ backgrounds are modeled using $\mathrm{MC}$ simulation and normalized to their respective NLO cross sections computed with MCFM 6.8 [68]. Other backgrounds, including $t \bar{t}, t W, W W, Z \rightarrow \tau \tau$, and DY, are estimated from data for the final selection. The background from $W+$ jets is negligible in the muon channel but significant in the electron channel, where an estimation method based on control samples in data is used for its estimation.

The background processes that do not involve $Z$ boson production are referred to as nonresonant backgrounds. Such backgrounds arise mainly from leptonic $W$ boson decays in $t \bar{t}, t W$, and $W W$ events. There are also small contributions from $s$ - and $t$-channel single top quark events and $Z \rightarrow \tau \tau$ events in which $\tau$ leptons produce electrons or muons and $E_{\mathrm{T}}^{\mathrm{miss}}$. We estimate these nonresonant backgrounds using a data control sample, consisting of events with an opposite-charge different-flavor dilepton pair $\left(e^{ \pm} \mu^{\mp}\right)$ that otherwise pass the full selection. As the decay rates for $Z \rightarrow e^{+} e^{-}$and $Z \rightarrow \mu^{+} \mu^{-}$are equal, by equating the ratio of observed dilepton counts to the square of the ratio of efficiencies, the backgrounds in the $e e$ and $\mu \mu$ channels can be estimated,

$$
\begin{array}{ll}
N_{\mathrm{bkg}, e e}^{\text {est }}=N_{e \mu}^{\mathrm{data}, \mathrm{corr}} k_{e e}, & k_{e e}=\frac{1}{2} \sqrt{\frac{N_{e e}^{\mathrm{data}}}{N_{\mu \mu}^{\mathrm{data}}}}, \\
N_{\mathrm{bkg}, \mu \mu}^{\mathrm{est}}=N_{e \mu}^{\mathrm{data}, \mathrm{corr}} k_{\mu \mu}, & k_{\mu \mu}=\frac{1}{2} \sqrt{\frac{N_{\mu \mu}^{\mathrm{data}}}{N_{e e}^{\mathrm{data}}}},
\end{array}
$$

in which the coefficient of $1 / 2$ in the correction factors $k_{e e}$ and $k_{\mu \mu}$ comes from the dilepton decay ratios for $e e, \mu \mu$, and $e \mu$ in these nonresonant backgrounds and $N_{e e}^{\text {data }}$ and $N_{\mu \mu}^{\text {data }}$ are the numbers of selected $e e$ and $\mu \mu$ events from data with masses inside the $Z$ mass window. The ratio $\sqrt{N_{e e}^{\text {data }} / N_{\mu \mu}^{\text {data }}}$ and the reciprocal quantity take into account the difference between the electron and muon selection efficiencies. The term $N_{e \mu}^{\mathrm{data}, \text { corr }}$ is the number of $e \mu$ events observed in data corrected by subtracting $Z Z, W Z$, DY, and $W+$ jets background contributions estimated using $\mathrm{MC}$ simulation. The kinematic distributions of the estimated nonresonant backgrounds are taken from the distributions of the $e \mu$ sample with the overall normalization determined by the method described above. The validity of this procedure for predicting nonresonant backgrounds is checked with simulated events containing $t \bar{t}, t W, W W$, and $Z \rightarrow \tau \tau$ processes. We assign a systematic uncertainty of $17 \%(15 \%)$ to this background estimation in the electron (muon) channel, based on closure tests that compare the predictions obtained from the control sample with those from the simulated events.

The DY process is dominant in the region of low $E_{\mathrm{T}}^{\text {miss }}$. This process does not produce undetectable particles, and therefore the measured $E_{\mathrm{T}}^{\text {miss }}$ arises from limited detector acceptance and mismeasurement. The estimation of this background uses simulated DY events, which are normalized to the data with scale factors obtained by measuring the number of DY events in background-dominated control regions, after subtracting other processes. These scale factors are of order 1.1-1.2. The control regions are defined with the full selection except for the requirements on $E_{\mathrm{T}}^{\text {miss }}$, $\Delta \phi_{\ell \ell, \vec{p}_{\mathrm{T}}^{\text {miss }}}$, and $\left|E_{\mathrm{T}}^{\text {miss }}-p_{\mathrm{T}}^{\ell \ell}\right| / p_{\mathrm{T}}^{\ell \ell}$. The results are calculated independently for control regions with variables $E_{\mathrm{T}}^{\text {miss }}$ and $\left|E_{\mathrm{T}}^{\mathrm{miss}}-p_{\mathrm{T}}^{\ell \ell}\right| / p_{\mathrm{T}}^{\ell \ell}$ and compared with each other as part of the estimate of systematic uncertainty. Based on the variations of the estimates with the choice of control regions, a systematic uncertainty of $10 \%(11 \%)$ is assigned to the DY background estimate in the electron (muon) channel.

A $W+$ jets background event consists of a genuine prompt lepton from the $W$ decay and a nonisolated lepton resulting from the leptonic decay of heavy quarks, 
TABLE II. Summary of systematic uncertainties. Each background uncertainty represents the variation of the relative yields of the particular background components. The signal uncertainties represent the relative variations in the signal acceptance, and ranges quoted cover both signals of DM and unparticles with different DM masses or scaling dimensions. For shape uncertainties, the numbers correspond to the overall effect of the shape variation on yield or acceptance. The symbol $\ldots$ indicates that the systematic uncertainty is not applicable.

\begin{tabular}{lcc}
\hline \hline Source & Background uncertainty $(\%)$ & Signal uncertainty $(\%)$ \\
\hline PDF $+\alpha_{S}$ & $5-6$ & $8-20$ \\
Factorization and renormalization scale & $7-8$ & 5 \\
Acceptance $(Z Z)$ & 14 & $\ldots$ \\
Integrated luminosity & 2.6 & 2.6 \\
Lepton trigger, reconstruction \& identification, isolation & 3 & 3 \\
DY normalization & $10-11$ & $\ldots$ \\
$t \bar{t}, t W, W W$ normalization & $15-17$ & $\ldots$ \\
$W+$ jets normalization & $15-23$ & $\ldots$ \\
MC statistics (signal, $Z Z, W Z)$ & $1-2$ & $\ldots$ \\
Control region statistics $(\mathrm{DY})$ & 25 & $\ldots$ \\
Control region statistics $(t \bar{t}, t W, W W$ ) & 18 & $\ldots$ \\
Control region statistics $(W+$ jets) & 36 & $0.1-0.7$ \\
Pileup & $0.5-1$ & $0.6-1$ \\
$b$-jet tagging efficiency & $0.4-1.4$ & $0.1-1$ \\
Lepton momentum scale & $0.4-0.5$ & $3-5$ \\
Jet energy scale and resolution & $5-7$ & 1 \\
Unclustered $E_{\mathrm{T}}^{\text {miss scale }}$ & $1-2$ & $\ldots$ \\
\hline \hline
\end{tabular}

misidentified hadrons, or electrons from photon conversions. The rate at which jets are misidentified as leptons may not be accurately described in the MC simulation, so the rate of jets passing lepton identification requirements is determined using a control data sample enriched in jets. The genuine lepton contamination from $W / Z+$ jets events in the selected control sample is subtracted using simulation to avoid biasing the calculation of the misidentification rate. The final estimation is obtained by applying these weights to a sample selected with lepton identification requirements that are looser than for the signal sample. The main source of systematic uncertainty for this background estimation comes from the measurement of the misidentification rate, which depends on the accuracy of the subtraction of real leptons in the control sample. A systematic uncertainty of $15 \%$ is assigned, based on the dependence of the calculated misidentification rates on the selection criteria applied to the control sample.

\section{EFFICIENCIES AND SYSTEMATIC UNCERTAINTIES}

The efficiencies for selecting, reconstructing, and identifying isolated leptons are determined from simulation and then corrected with scale factors determined from applying a "tag-and-probe" technique [69] to $Z \rightarrow \ell^{+} \ell^{-}$events. The

TABLE III. Signal predictions, background estimates, and observed number of events. The DM signal yields are given for masses $m_{\chi}=10,200$, and $500 \mathrm{GeV}$ and cutoff scales $\Lambda=0.37,0.53,0.48$, and $1.4 \mathrm{TeV}$. The yields from an unparticle signal are presented with a scaling dimension $d_{\mathcal{U}}=1.6$ and a renormalization scale $\Lambda_{\mathcal{U}}=33 \mathrm{TeV}$. The corresponding statistical and systematic uncertainties are shown, in that order.

\begin{tabular}{lcc}
\hline \hline Process & $e^{+} e^{-}$ & $\mu^{+} \mu^{-}$ \\
\hline $\mathrm{C} 3, m_{\chi}=10 \mathrm{GeV}, \Lambda=0.37 \mathrm{TeV}$ & $10.7 \pm 0.2 \pm 1.1$ & $12.8 \pm 0.3 \pm 1.1$ \\
$\mathrm{D} 5, m_{\chi}=10 \mathrm{GeV}, \Lambda=0.53 \mathrm{TeV}$ & $10.0 \pm 0.3 \pm 1.1$ & $12.8 \pm 0.3 \pm 1.1$ \\
$\mathrm{D} 8, m_{\chi}=200 \mathrm{GeV}, \Lambda=0.48 \mathrm{TeV}$ & $9.0 \pm 0.2 \pm 1.1$ & $11.1 \pm 0.2 \pm 0.9$ \\
$\mathrm{D} 9, m_{\chi}=500 \mathrm{GeV}, \Lambda=1.4 \mathrm{TeV}$ & $2.67 \pm 0.03 \pm 0.41$ & $2.81 \pm 0.03 \pm 0.26$ \\
$\mathrm{Unparticle}, d_{\mathcal{U}}=1.6, \Lambda_{\mathcal{U}}=33 \mathrm{TeV}$ & $19.0 \pm 0.3 \pm 1.3$ & $25.6 \pm 0.4 \pm 1.7$ \\
$Z / \gamma^{*} \rightarrow \ell^{+} \ell^{-}$ & $8.2 \pm 1.9 \pm 0.8$ & $8.6 \pm 3.0 \pm 1.0$ \\
$W Z \rightarrow 3 \ell \nu$ & $25.1 \pm 0.5 \pm 2.8$ & $40.7 \pm 0.7 \pm 4.5$ \\
$Z Z \rightarrow 2 \ell 2 \nu$ & $59 \pm 1 \pm 10$ & $79 \pm 1 \pm 14$ \\
$t \bar{t} / t W / W W / Z \rightarrow \tau \tau$ & $18.7 \pm 3.4 \pm 3.3$ & $22.9 \pm 2.3 \pm 3.4$ \\
$W+$ jets & $1.8 \pm 0.6 \pm 0.3$ & $151 \pm 4 \pm 18$ \\
Total background & $113 \pm 4 \pm 13$ & 133 \\
Data & 111 & \\
\hline \hline
\end{tabular}


trigger efficiencies for the electron and muon channels are found to be above $90 \%$, varying as a function of $p_{\mathrm{T}}$ and $|\eta|$ of the lepton. The identification efficiency for electrons (muons), when applying the criteria described in Sec. IV, is found to be $95 \%$ (94\%). The corresponding data-to-MC scale factors are typically in the range 0.94-1.01 (0.981.02) for the electron (muon) channel, depending on the $p_{T}$ and $|\eta|$ of the lepton candidate. For both channels, the overall uncertainty in selecting and reconstructing leptons in an event is about $3 \%$.

The systematic uncertainties include normalization uncertainties that affect the overall size of contributions and shape uncertainties that alter the shapes of the distributions used in extracting the signal limits. The systematic uncertainties are summarized in Table II.

The normalization uncertainties in the background estimates from data are described in Sec. VI. The overall approach for the estimation of the PDF and $\alpha_{S}$ uncertainties (referred to as $\mathrm{PDF}+\alpha_{S}$ in the following) adopts the interim recommendations of the PDF4LHC group and is used both for signal and the background [70-74]. This is the most important uncertainty for the signals. As the mass of the DM particles increases, the $\mathrm{PDF}+\alpha_{S}$ uncertainty reaches $20 \%$, which can be explained by the diminishing phase space for DM production and the rise of the corresponding uncertainty in the cross section. The efficiencies for signal, $Z Z$, and $W Z$ processes are estimated using simulation, and the uncertainties in the corresponding yields are derived from variations of the renormalization and factorization scales, $\alpha_{S}$, and choice of PDFs, in which the factorization and renormalization scales are assessed by varying the original scales of the process by factors of 0.5 and 2. Typical values for the signal extraction efficiency are found to be around $40 \%$. The uncertainty related to the renormalization and factorization scales is $5 \%$ for signal and 7\%-8\% for $Z Z$ and $W Z$ processes. The effect of variations in $\alpha_{S}$ and the choice of PDFs is 5\%-6\% for the $Z Z$ and $W Z$ backgrounds. The uncertainty assigned to the luminosity measurement is $2.6 \%$ [38].

The contributions to the shape uncertainties come from the lepton momentum scale, the jet energy scale and resolution, the unclustered $E_{\mathrm{T}}^{\text {miss }}$ scale, the $b$ tagging efficiency, and the pileup modeling. Each corresponding uncertainty is calculated by varying the respective variable of interest within its own uncertainties and propagating the variations to the variable $m_{\mathrm{T}}$ using the final selection. In the case of the lepton momentum scale, the uncertainty is computed by varying the momentum of the leptons by their uncertainties. The uncertainty in the muon momentum scale is $1 \%$. For electrons, uncertainties of $0.6 \%$ for the barrel and $1.5 \%$ for the end caps are applied. For the $Z Z$ background, cross checks are made using the generators MADGRAPH, POWHEG [75], and SHERPA 2.1.1 [76]. A comparison of the acceptance from normalized yields obtained with these generators is made in the signal region.
In each bin of $m_{\mathrm{T}}$, the maximum difference in acceptance with respect to the MADGRAPH prediction is assigned as a separate additional systematic uncertainty. The limit setting procedure discussed in Sec. VIII incorporates this acceptance uncertainty from each of the separate bins. The weighted average of these uncertainties is $14 \%$. This is the dominant uncertainty in the total background prediction for the signal region. For the $W Z$ background, this difference in acceptance is not observed, and data and simulation agree in the selected three-lepton control region.
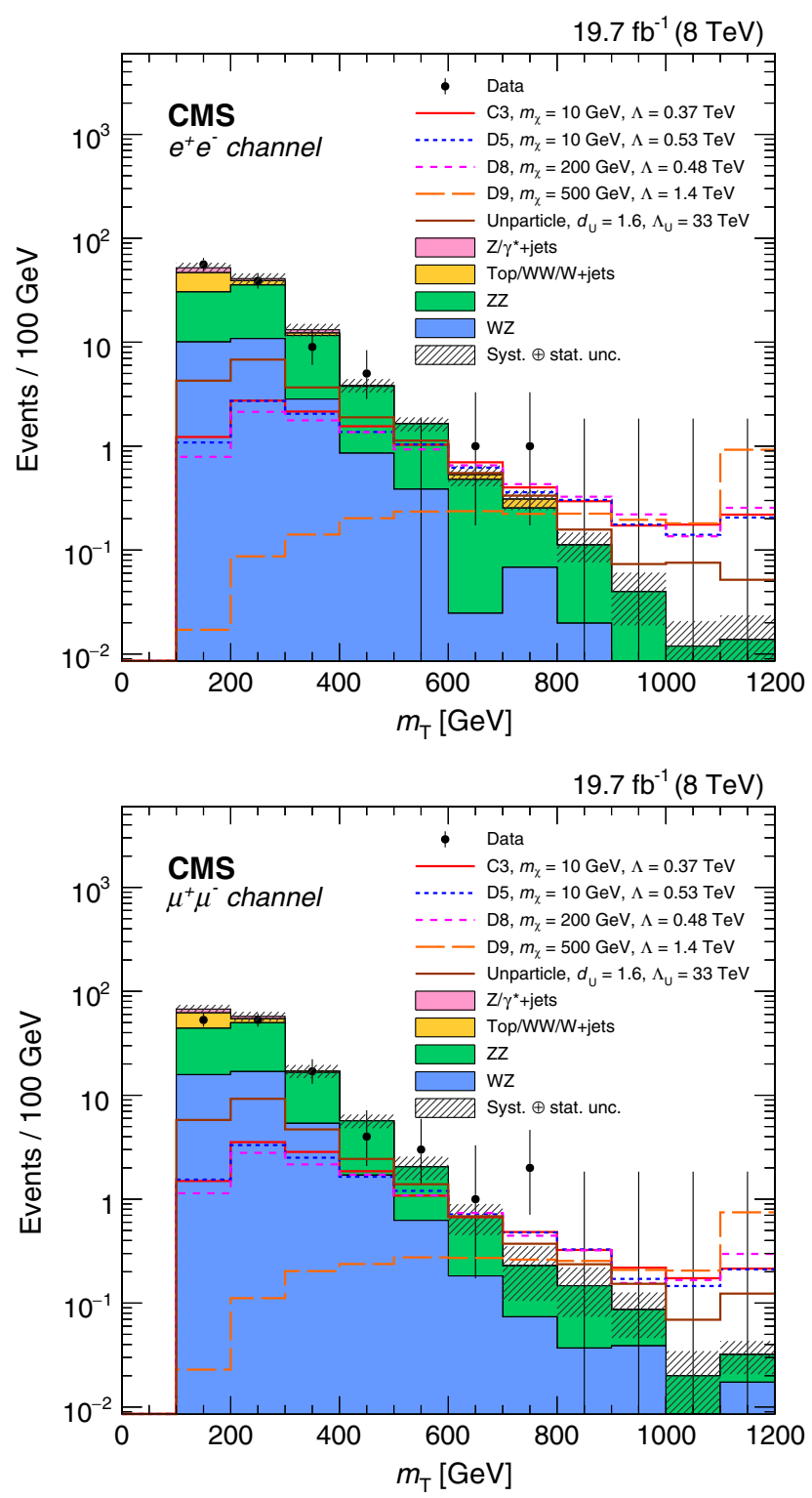

FIG. 5. Distributions of the transverse mass for the final selection in the $e^{+} e^{-}$(left) and $\mu^{+} \mu^{-}$(right) channels. Examples of expected signal distributions are shown for DM particle production and unparticle production. The total statistical and systematic uncertainty in the overall background is shown as a hatched region. Overflow events are included in the rightmost bins. 
The uncertainties in the calibration of the jet energy scale and resolution directly affect the assignments of jets to jet categories, the $E_{\mathrm{T}}^{\text {miss }}$ computation, and all the selections related to jets. The effect of the jet energy scale uncertainty is estimated by varying the energy scale by $\pm 1 \sigma$. A similar strategy is used to evaluate the systematic uncertainty related to the jet energy resolution. The uncertainties in the final yields are found to be 3\%-5\% (5\%-7\%) for signal (background). The effect of the uncertainty in the energy scale of the unclustered component of the $E_{\mathrm{T}}^{\text {miss }}$ measurement is estimated by subtracting the leptons and jets from the $E_{\mathrm{T}}^{\text {miss }}$ summation and by varying the residual recoil by $\pm 10 \%$. The clustered component is then added back in order to recalculate the value of $E_{\mathrm{T}}^{\text {miss }}$. The resultant uncertainty in the final yields is found to be of order
$1 \%-2 \%$. Since the $b$ tagging efficiencies measured in data are somewhat different from those predicted by the simulation, an event-by-event reweighting using data-to-MC scale factors is applied to simulated events. The uncertainty associated with this procedure is obtained by varying the event-by-event weight by $\pm 1 \sigma$. The total uncertainty in the final yields is $0.6 \%-1 \%(0.4 \%-1.4 \%)$ for signal (background). All simulated events are reweighted to reproduce the pileup conditions observed in data. To compute the uncertainty related to pileup modeling, we shift the mean of the distribution in simulation by $5 \%$. The variation of the final yields induced by this procedure is less than $1 \%$. For the processes estimated from simulation, the sizes of the MC samples limit the precision of the modeling, and the corresponding statistical uncertainty is incorporated into
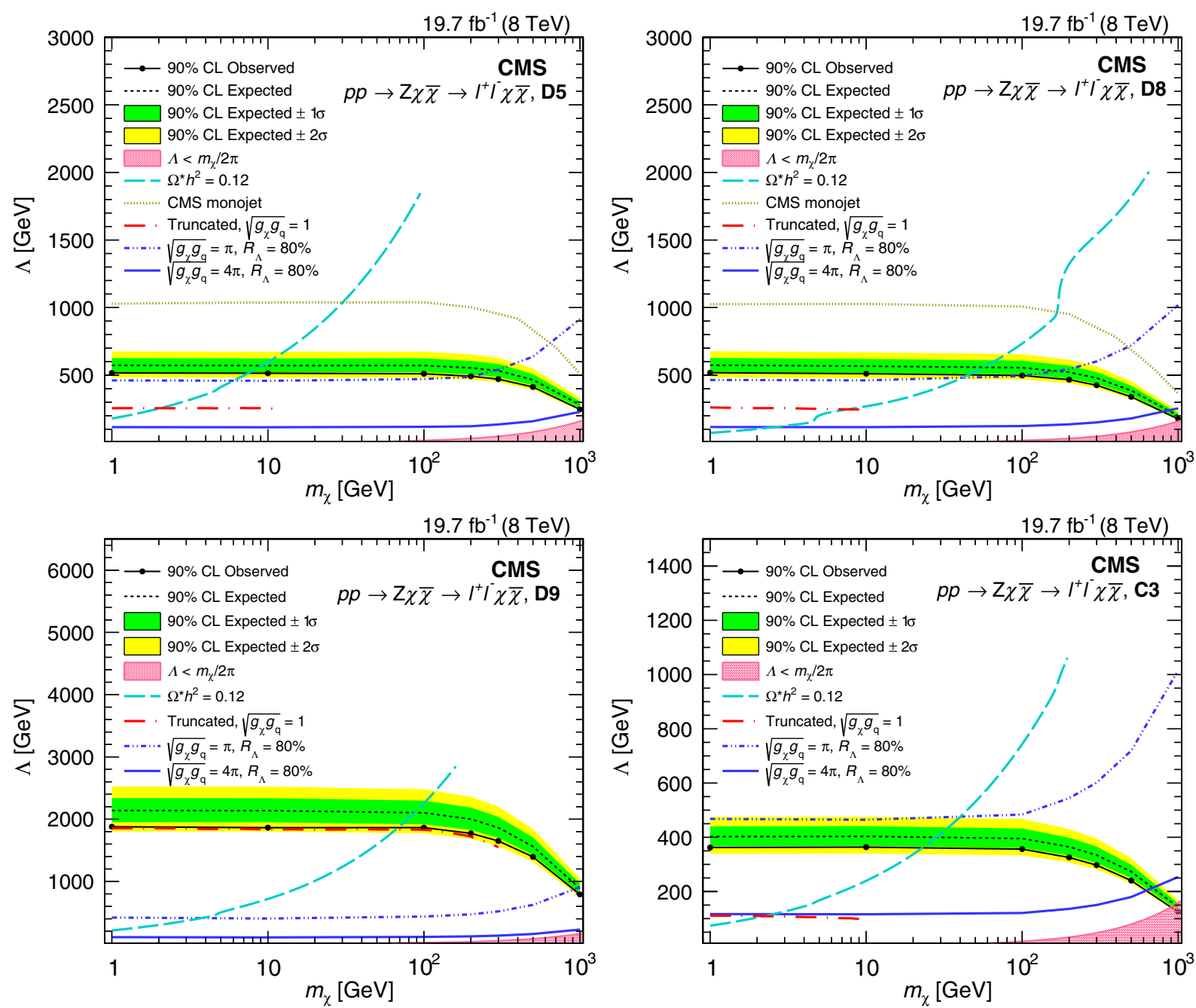

FIG. 6. Expected and observed 90\% CL lower limits on $\Lambda$ as a function of DM particle mass $m_{\chi}$ for the operators D5 (top left), D8 (top right), D9 (bottom left), and C3 (bottom left). The pink shaded area is shown in each plot to indicate the lower bound $\Lambda>m_{\chi} / 2 \pi$ on the validity of the effective field theory DM model. The cyan long-dashed line calculated by MadDM 1.0 [82] reflects the relic density of cold, nonbaryonic DM: $\Omega h^{2}=0.1198 \pm 0.0026$ measured by the Planck telescope [81]. Monojet results from CMS [14] are shown for comparison. Truncated limits with $\sqrt{g_{q} g_{\chi}}=1$ are presented with red dot long-dashed lines. The blue double-dot and triple-dot dashed lines indicate the contours of $R_{\Lambda}=80 \%$ for all operators with couplings $\sqrt{g_{\mathrm{q}} g_{\chi}}=\pi$ and $4 \pi$. 
the shape uncertainty. A similar treatment is applied to the backgrounds estimated from control samples in data based on the statistical uncertainties in the corresponding control samples.

\section{RESULTS}

For both the electron and the muon channels, a shapebased analysis is employed. The expected numbers of background and signal events scaled by a signal strength modifier are combined in a binned likelihood for each bin of the $m_{\mathrm{T}}$ distribution. The signal strength modifier, defined as the signal cross section divided by the cross section suggested by theory, determines the strength of the signal process. The numbers of observed and expected events are shown in Table III, including the expectation for a selected mass point for each type of signal. Figure 5 shows the $m_{\mathrm{T}}$ distributions after the final selection. The observed distributions agree with the SM background predictions, and no excess of events is observed.

Upper limits on the contribution of events from new physics are computed by using the modified frequentist approach $\mathrm{CL}_{\mathrm{s}}[77,78]$ based on asymptotic formulas $[79,80]$.

\section{A. DM interpretation}

The observed limit on the cross section for DM production depends on the DM particle mass and the nature of DM interactions with SM particles. Within the framework of effective field theory, the upper limits on this cross section can be translated into 90\% CL lower limits on the effective cutoff scale $\Lambda$ as a function of DM particle mass $m_{\chi}$, as shown in Fig. 6. The choice of $90 \% \mathrm{CL}$ is made in order to allow comparisons with direct detection experiments. The relic density of cold, nonbaryonic DM has been measured by Planck telescope [81] using the anisotropy of the cosmic microwave background and of the spatial distribution of galaxies. They obtain a value $\Omega h^{2}=0.1198 \pm 0.0026$, where $h$ is the Hubble constant. The implications of this result plotted in the plane of the effective cutoff scale $\Lambda$ and DM mass $m_{\chi}$ have been calculated with MadDM 1.0 [82] and are shown in Fig. 6. Results from a search for DM particles using monojet signatures in CMS [14] are also plotted for comparison.

It has been emphasized by several authors [28,83-85] that the effective field theory approach is not valid over the full range of phase space that is accessible at the LHC, since the scales involved can be comparable to the collision energy. In the LHC regime, the assumption of a pointlike interaction provides a reliable approximation of the underlying ultraviolet-complete theory only for appropriate choices of couplings and masses. To estimate the region of validity relevant to this analysis, we consider a simple tree-level ultraviolet-complete model that contains a massive mediator $(M)$ exchanged in the $s$ channel, with the couplings to quarks and DM particles described by coupling constants $g_{q}$ and $g_{\chi}$. The effective cutoff scale $\Lambda$ thus can be expressed as $\Lambda \sim M / \sqrt{g_{q} g_{\chi}}$, when momentum transfer is small $\left(Q_{\mathrm{tr}}<M\right)$. Imposing a condition on the couplings $\sqrt{g_{q} g_{\chi}}<4 \pi$ to ensure stability of the perturbative calculation and a mass requirement $M>2 m_{\chi}$, a lower
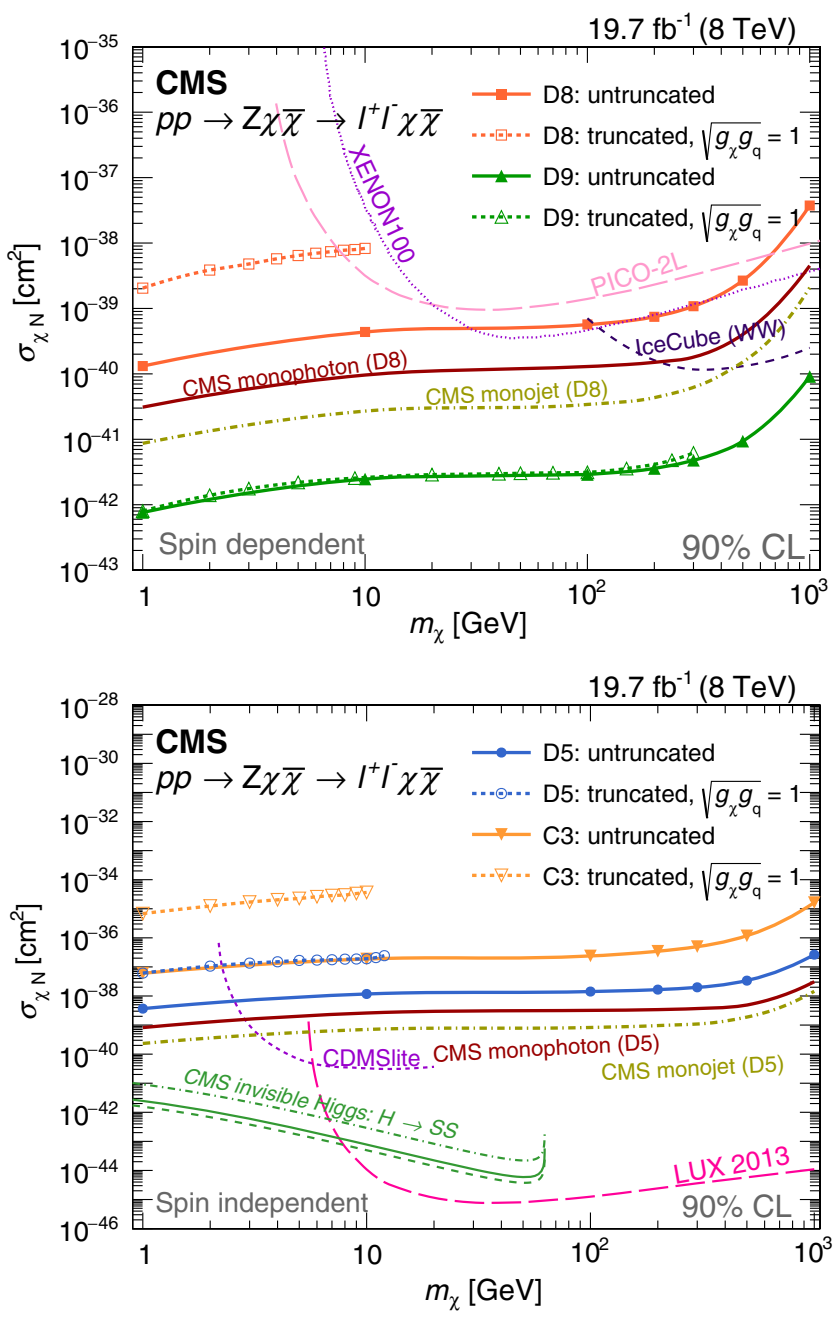

FIG. 7. The 90\% CL upper limits on the DM-nucleon cross section as a function of the DM particle mass. Left: spindependent limits for axial-vector (D8) and tensor (D9) coupling of Dirac fermion DM candidates, together with direct search experimental results from the PICO [101], XENON100 [102], and IceCube [7] collaborations. Right: spin-independent limits for vector coupling of complex scalar (C3) and Dirac fermion (D5) DM candidates, together with CDMSlite [8], LUX [11], as well as Higgs-portal scalar DM results from CMS [96] with central (solid), minimum (dashed), and maximum (dot dashed) values of Higgs-nucleon couplings. Collider results from CMS monojet [14] and monophoton [16] searches, interpreted in both spin-dependent and spin-independent scenarios, are shown for comparison. The truncated limits for D5, D8, D9, and C3 with $\sqrt{g_{q} g_{\chi}}=1$ are presented with dashed lines in same shade as the untruncated ones. 
bound $\Lambda>m_{\chi} / 2 \pi$ is obtained for the region of validity. The area below this boundary, where the effective theory of $\mathrm{DM}$ is not expected to provide a reliable prediction at the LHC, is shown as a pink shaded area in each of the panels of Fig. 6.

However, the requirement of $\Lambda>m_{\gamma} / 2 \pi$ is not sufficient, according to some authors [83,85-94], and the region of validity depends on the coupling values in the ultraviolet completion of the theory. Considering a more realistic minimum constraint $Q_{\mathrm{tr}}<M \sim \sqrt{g_{q} g_{\chi}} \Lambda$, we can calculate the ratio $R_{\Lambda}$ of the number of events fulfilling the validity criteria over all events produced in the accessible phase space,

$$
R_{\Lambda}=\frac{\left.\int_{p_{\mathrm{T}}^{\min }}^{p_{\max }^{\max }} d p_{\mathrm{T}} \int_{\eta^{\min }}^{m_{\max }^{\max }} d \eta \frac{d^{2} \sigma_{\text {eff }}}{d p_{\mathrm{T}} d \eta}\right|_{Q_{\mathrm{tr}}<\sqrt{g_{q} g_{\chi}} \Lambda}}{\int_{p_{\mathrm{T}}^{\min }}^{p_{\operatorname{mix}}^{\operatorname{mix}}} d p_{\mathrm{T}} \int_{\eta^{\min }}^{\eta^{\max }} d \eta \frac{d^{2} \sigma_{\text {eff }}}{d p_{\mathrm{T}} d \eta}}
$$

in which the values of $R_{\Lambda}$ can be used to check the accuracy of the effective description in regions of parameter space $\left(\Lambda, m_{\chi}\right)$. Figure 6 includes the corresponding contours of $R_{\Lambda}=80 \%$ for all operators with couplings $\sqrt{g_{q} g_{\chi}}=\pi$ and $4 \pi$. Alternatively, we can obtain the truncated limits by manually removing the events with $Q_{\mathrm{tr}}>\sqrt{g_{q} g_{\chi}} \Lambda$ at the generator level. Figure 6 also shows these truncated limits with $\sqrt{g_{q} g_{\chi}}=1$. For a certain value of $m_{\chi}$, the truncated limit goes to zero quickly because none of the events above this value fulfills the requirement $Q_{\mathrm{tr}}<\sqrt{g_{q} g_{\chi}} \Lambda$. For a maximum coupling $g_{\chi, q}=4 \pi, 100 \%$ of the events passes this requirement, and the truncated limits coincide with the observed one and are not shown.

Figure 7 shows the 90\% CL upper limits on the DMnucleon cross section as a function of DM particle mass for both the spin-dependent and spin-independent cases $[83,95]$ obtained using the relations

$$
\begin{aligned}
\sigma_{0}^{\mathrm{D} 8, \mathrm{D} 9} & =\sum_{\mathrm{q}} \frac{3 \mu_{\chi N}^{2}}{\pi \Lambda^{4}}\left(\Delta_{q}^{N}\right)^{2} \\
& =9.18 \times 10^{-40} \mathrm{~cm}^{2}\left(\frac{\mu_{\chi N}}{1 \mathrm{GeV}}\right)^{2}\left(\frac{300 \mathrm{GeV}}{\Lambda}\right)^{4}, \\
\sigma_{0}^{\mathrm{D} 5} & =\sum_{\mathrm{q}} \frac{\mu_{\chi N}^{2}}{\pi \Lambda^{4}}\left(f_{\mathrm{q}}^{N}\right)^{2} \\
& =1.38 \times 10^{-37} \mathrm{~cm}^{2}\left(\frac{\mu_{\chi N}}{1 \mathrm{GeV}}\right)^{2}\left(\frac{300 \mathrm{GeV}}{\Lambda}\right)^{4}, \\
\sigma_{0}^{\mathrm{C} 3} & =\sum_{\mathrm{q}} \frac{4 \mu_{\chi N}^{2}}{\pi \Lambda^{4}}\left(f_{\mathrm{q}}^{N}\right)^{2} \\
& =5.52 \times 10^{-37} \mathrm{~cm}^{2}\left(\frac{\mu_{\chi N}}{1 \mathrm{GeV}}\right)^{2}\left(\frac{300 \mathrm{GeV}}{\Lambda}\right)^{4},
\end{aligned}
$$

TABLE IV. Expected and observed 90\% CL upper limits on the DM-nucleon cross section $\sigma_{\chi N}$ and effective cutoff scale $\Lambda$ for operator D5.

\begin{tabular}{lcccccccc}
\hline \hline$m_{\chi}$ & \multicolumn{2}{c}{ Expected } & \multicolumn{2}{c}{ Expected $-1 \sigma$} & \multicolumn{2}{c}{ Expected $+1 \sigma$} & \multicolumn{2}{c}{ Observed } \\
\hline$(\mathrm{GeV})$ & $\Lambda(\mathrm{GeV})$ & $\sigma_{\chi N}\left(\mathrm{~cm}^{2}\right)$ & $\Lambda(\mathrm{GeV})$ & $\sigma_{\chi N}\left(\mathrm{~cm}^{2}\right)$ & $\Lambda(\mathrm{GeV})$ & $\sigma_{\chi N}\left(\mathrm{~cm}^{2}\right)$ & $\Lambda(\mathrm{GeV})$ & $\sigma_{\chi N}\left(\mathrm{~cm}^{2}\right)$ \\
\hline 1 & 572 & $2.4 \times 10^{-39}$ & 626 & $1.7 \times 10^{-39}$ & 522 & $3.5 \times 10^{-39}$ & 516 & $3.7 \times 10^{-39}$ \\
10 & 570 & $7.8 \times 10^{-39}$ & 624 & $5.4 \times 10^{-39}$ & 520 & $1.1 \times 10^{-38}$ & 514 & $1.2 \times 10^{-38}$ \\
100 & 571 & $9.1 \times 10^{-39}$ & 625 & $6.3 \times 10^{-39}$ & 521 & $1.3 \times 10^{-38}$ & 510 & $1.4 \times 10^{-38}$ \\
200 & 554 & $1.0 \times 10^{-38}$ & 606 & $7.2 \times 10^{-39}$ & 505 & $1.5 \times 10^{-38}$ & 492 & $1.7 \times 10^{-38}$ \\
300 & 533 & $1.2 \times 10^{-38}$ & 583 & $8.5 \times 10^{-39}$ & 486 & $1.7 \times 10^{-38}$ & 471 & $2.0 \times 10^{-38}$ \\
500 & 465 & $2.1 \times 10^{-38}$ & 509 & $1.5 \times 10^{-38}$ & 425 & $3.0 \times 10^{-38}$ & 413 & $3.4 \times 10^{-38}$ \\
1000 & 281 & $1.6 \times 10^{-37}$ & 308 & $1.1 \times 10^{-37}$ & 257 & $2.3 \times 10^{-37}$ & 247 & $2.6 \times 10^{-37}$ \\
\hline \hline
\end{tabular}

TABLE V. Expected and observed 90\% CL upper limits on the DM-nucleon cross section $\sigma_{\chi^{N}}$ and effective cutoff scale $\Lambda$ for operator D8.

\begin{tabular}{lcccccccc}
\hline \hline$m_{\chi}$ & \multicolumn{2}{c}{ Expected } & \multicolumn{2}{c}{ Expected $-1 \sigma$} & \multicolumn{2}{c}{ Expected $+1 \sigma$} & \multicolumn{2}{c}{ Observed } \\
\hline$(\mathrm{GeV})$ & $(\mathrm{GeV}) \Lambda$ & $\sigma_{\chi N}\left(\mathrm{~cm}^{2}\right)$ & $\Lambda(\mathrm{GeV})$ & $\sigma_{\chi N}\left(\mathrm{~cm}^{2}\right)$ & $\Lambda(\mathrm{GeV})$ & $\sigma_{\chi N}\left(\mathrm{~cm}^{2}\right)$ & $\Lambda(\mathrm{GeV})$ & $\sigma_{\chi N}\left(\mathrm{~cm}^{2}\right)$ \\
\hline 1 & 574 & $8.8 \times 10^{-41}$ & 627 & $6.1 \times 10^{-41}$ & 523 & $1.3 \times 10^{-40}$ & 518 & $1.3 \times 10^{-40}$ \\
10 & 567 & $2.9 \times 10^{-40}$ & 620 & $2.0 \times 10^{-40}$ & 517 & $4.2 \times 10^{-40}$ & 511 & $4.4 \times 10^{-40}$ \\
100 & 555 & $3.7 \times 10^{-40}$ & 607 & $2.6 \times 10^{-40}$ & 507 & $5.3 \times 10^{-40}$ & 498 & $5.7 \times 10^{-40}$ \\
200 & 522 & $4.8 \times 10^{-40}$ & 570 & $3.3 \times 10^{-40}$ & 476 & $6.9 \times 10^{-40}$ & 467 & $7.5 \times 10^{-40}$ \\
300 & 479 & $6.7 \times 10^{-40}$ & 524 & $4.7 \times 10^{-40}$ & 437 & $9.7 \times 10^{-40}$ & 425 & $1.1 \times 10^{-39}$ \\
500 & 386 & $1.6 \times 10^{-39}$ & 422 & $1.1 \times 10^{-39}$ & 352 & $2.3 \times 10^{-39}$ & 340 & $2.7 \times 10^{-39}$ \\
1000 & 199 & $2.3 \times 10^{-38}$ & 218 & $1.6 \times 10^{-38}$ & 182 & $3.3 \times 10^{-38}$ & 176 & $3.7 \times 10^{-38}$ \\
\hline \hline
\end{tabular}


TABLE VI. Expected and observed 90\% CL upper limits on the DM-nucleon cross section $\sigma_{\chi N}$ and effective cutoff scale $\Lambda$ for operator D9.

\begin{tabular}{|c|c|c|c|c|c|c|c|c|}
\hline \multirow{2}{*}{$\frac{m_{\chi}}{(\mathrm{GeV})}$} & \multicolumn{2}{|c|}{ Expected } & \multicolumn{2}{|c|}{ Expected $-1 \sigma$} & \multicolumn{2}{|c|}{ Expected $+1 \sigma$} & \multicolumn{2}{|c|}{ Observed } \\
\hline & $\Lambda(\mathrm{GeV})$ & $\sigma_{\chi N}\left(\mathrm{~cm}^{2}\right)$ & $\Lambda(\mathrm{GeV})$ & $\sigma_{\chi N}\left(\mathrm{~cm}^{2}\right)$ & $\Lambda(\mathrm{GeV})$ & $\sigma_{\chi N}\left(\mathrm{~cm}^{2}\right)$ & $\Lambda(\mathrm{GeV})$ & $\sigma_{\chi N}\left(\mathrm{~cm}^{2}\right)$ \\
\hline 1 & 2139 & $4.5 \times 10^{-43}$ & 2339 & $3.2 \times 10^{-43}$ & 1951 & $6.5 \times 10^{-43}$ & 1879 & $7.6 \times 10^{-43}$ \\
\hline 10 & 2137 & $1.4 \times 10^{-42}$ & 2337 & $1.0 \times 10^{-42}$ & 1950 & $2.1 \times 10^{-42}$ & 1864 & $2.5 \times 10^{-42}$ \\
\hline 100 & 2102 & $1.8 \times 10^{-42}$ & 2299 & $1.3 \times 10^{-42}$ & 1918 & $2.6 \times 10^{-42}$ & 1865 & $2.9 \times 10^{-42}$ \\
\hline 200 & 2000 & $2.2 \times 10^{-42}$ & 2187 & $1.5 \times 10^{-42}$ & 1825 & $3.2 \times 10^{-42}$ & 1772 & $3.6 \times 10^{-42}$ \\
\hline 300 & 1863 & $2.9 \times 10^{-42}$ & 2038 & $2.1 \times 10^{-42}$ & 1700 & $4.2 \times 10^{-42}$ & 1650 & $4.8 \times 10^{-42}$ \\
\hline 500 & 1562 & $6.0 \times 10^{-42}$ & 1708 & $4.2 \times 10^{-42}$ & 1425 & $8.6 \times 10^{-42}$ & 1395 & $9.4 \times 10^{-42}$ \\
\hline 1000 & 886 & $5.8 \times 10^{-41}$ & 969 & $4.0 \times 10^{-41}$ & 809 & $8.3 \times 10^{-41}$ & 790 & $9.1 \times 10^{-41}$ \\
\hline
\end{tabular}

TABLE VII. Expected and observed 90\% CL upper limits on the DM-nucleon cross section $\sigma_{\chi_{N}}$ and effective cutoff scale $\Lambda$ for operator $\mathrm{C} 3$.

\begin{tabular}{|c|c|c|c|c|c|c|c|c|}
\hline \multirow{2}{*}{$\frac{m_{\chi}}{(\mathrm{GeV})}$} & \multicolumn{2}{|c|}{ Expected } & \multicolumn{2}{|c|}{ Expected $-1 \sigma$} & \multicolumn{2}{|c|}{ Expected $+1 \sigma$} & \multicolumn{2}{|c|}{ Observed } \\
\hline & $\Lambda(\mathrm{GeV})$ & $\sigma_{\chi N}\left(\mathrm{~cm}^{2}\right)$ & $\Lambda(\mathrm{GeV})$ & $\sigma_{\chi N}\left(\mathrm{~cm}^{2}\right)$ & $\Lambda(\mathrm{GeV})$ & $\sigma_{\chi N}\left(\mathrm{~cm}^{2}\right)$ & $\Lambda(\mathrm{GeV})$ & $\sigma_{\chi N}\left(\mathrm{~cm}^{2}\right)$ \\
\hline$\overline{1}$ & 403 & $4.0 \times 10^{-38}$ & 440 & $2.8 \times 10^{-38}$ & 367 & $5.7 \times 10^{-38}$ & 363 & $6.1 \times 10^{-38}$ \\
\hline 10 & 403 & $1.2 \times 10^{-37}$ & 441 & $8.7 \times 10^{-38}$ & 368 & $1.8 \times 10^{-37}$ & 364 & $1.9 \times 10^{-37}$ \\
\hline 100 & 396 & $1.6 \times 10^{-37}$ & 433 & $1.1 \times 10^{-37}$ & 361 & $2.3 \times 10^{-37}$ & 356 & $2.4 \times 10^{-37}$ \\
\hline 200 & 365 & $2.2 \times 10^{-37}$ & 399 & $1.5 \times 10^{-37}$ & 333 & $3.2 \times 10^{-37}$ & 326 & $3.5 \times 10^{-37}$ \\
\hline 300 & 335 & $3.1 \times 10^{-37}$ & 366 & $2.2 \times 10^{-37}$ & 305 & $4.5 \times 10^{-37}$ & 297 & $5.0 \times 10^{-37}$ \\
\hline 500 & 273 & $7.0 \times 10^{-37}$ & 299 & $4.9 \times 10^{-37}$ & 250 & $1.0 \times 10^{-36}$ & 241 & $1.2 \times 10^{-36}$ \\
\hline 1000 & 143 & $9.5 \times 10^{-36}$ & 156 & $6.6 \times 10^{-36}$ & 130 & $1.4 \times 10^{-35}$ & 125 & $1.6 \times 10^{-35}$ \\
\hline
\end{tabular}

where $\mu_{\chi N}$ is the reduced mass of the DM-nucleon system, $f_{q}^{N}$ characterizes the nucleon structure $\left(f_{u}^{p}=f_{d}^{n}=2\right.$ and $f_{d}^{p}=f_{u}^{n}=1 ; f=0$ otherwise), and $\Delta_{\mathrm{q}}^{N}$ represents a spin-dependent form factor $\left(\Delta_{u}^{p}=\Delta_{d}^{n}=0.842 \pm 0.012\right.$, $\left.\Delta_{d}^{p}=\Delta_{u}^{n}=-0.427 \pm 0.013, \quad \Delta_{s}^{p}=\Delta_{s}^{n}=-0.085 \pm 0.018\right)$ as specified in Ref. [95]. The truncated limits for D5, D8, D9, and C3 with $\sqrt{g_{q} g_{\chi}}=1$ are presented with dashed lines in the same shade as the untruncated ones. For comparison, direct search results as well as collider results from the CMS monojet [14] and monophoton [16] studies are shown. The recent limits from ATLAS [26] coincide very closely with the untruncated CMS limits on D9 and D5 operators. The ATLAS curves are not shown on the figure, since they would be difficult to distinguish by eye. Results are also shown from a search for the invisible decays of the Higgs boson [96], interpreted in a Higgsportal model [97,98], where a Higgs boson with a mass of $125 \mathrm{GeV}$ acts as a mediator between scalar DM and SM particles. The central (solid) line corresponds to the Higgsnucleon coupling value (0.326) from a lattice calculation [99], and the upper (dot-dashed) and lower (dashed) lines are maximum (0.629) and minimum (0.260) values from the MILC Collaboration [100].

The expected and observed limits on the effective cutoff scale $\Lambda$ as a function of the DM particle mass $m_{\chi}$ are listed in Tables IV and V for the operators D5 and

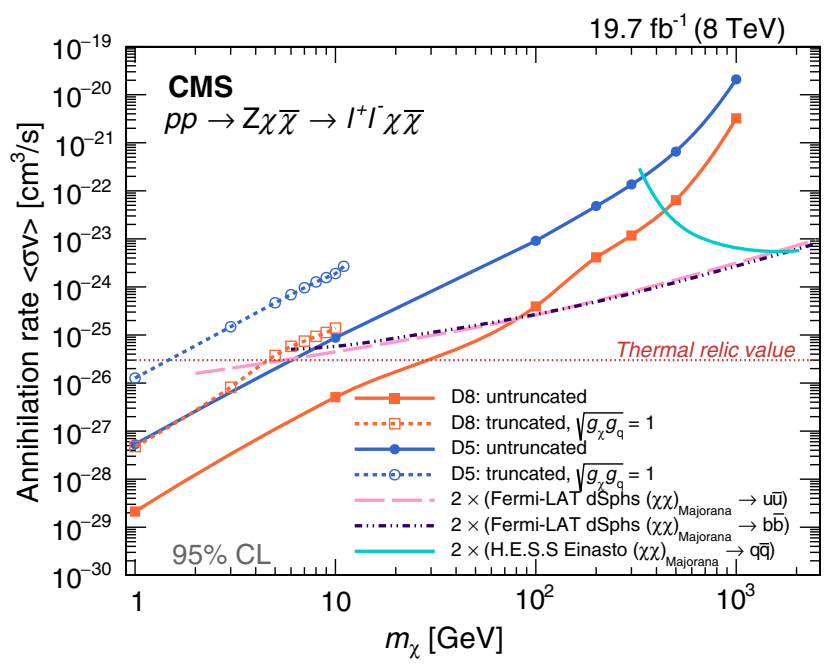

FIG. 8. The 95\% CL upper limits on the DM annihilation rate $\langle\sigma v\rangle$ for $\chi \bar{\chi} \rightarrow q \bar{q}$ as a function of the DM particle mass for vector (D5) and axial-vector (D8) couplings of Dirac fermion DM. A $100 \%$ branching fraction of DM annihilating to quarks is assumed. Indirect search experimental results from H.E.S.S [103] and Fermi-LAT [104] are also plotted. The value required for DM particles to account for the relic abundance is labeled "Thermal relic value" and is shown as a red dotted line. The truncated limits for D5 and D8 with $\sqrt{g_{q} g_{\chi}}=1$ are presented with dashed lines in same shade as the untruncated ones. 
D8. The values for the operators D9 and C3 are listed in Tables VI and VII. The results are also shown in terms of limits on DM-nucleon cross sections $\sigma_{\chi N}$, to allow comparison with the results from direct searches for DM particles.

Figure 8 shows the limits from operators D5 and D8 translated into upper limits on the DM annihilation rate $\langle\sigma v\rangle$ relevant to indirect astrophysical searches [86], in which $\sigma$ is the annihilation cross section, $v$ is the relative velocity of the annihilating particles, and the quantity $\langle\sigma v\rangle$ is averaged over the distribution of the DM velocity.
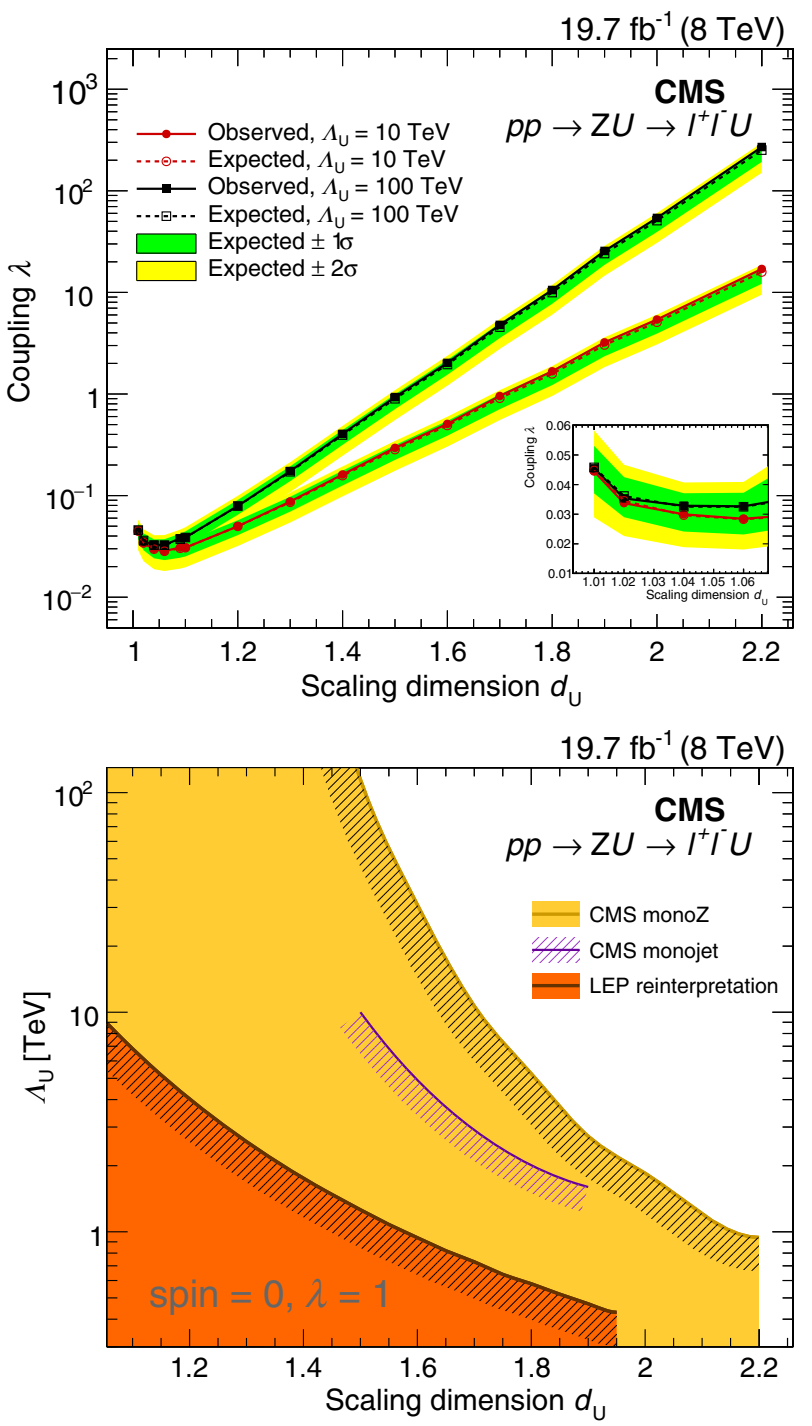

FIG. 9. Left: 95\% CL upper limits on the coupling $\lambda$ between the unparticle and SM fields with fixed effective cutoff scales $\Lambda_{\mathcal{U}}=10$ and $100 \mathrm{TeV}$. The plot inserted provides an expanded view of the limits at low scaling dimension. Right: 95\% CL lower limits on unparticle effective cutoff scale $\Lambda_{\mathcal{U}}$ with a fixed coupling $\lambda=1$. The results from CMS monojet [14] and reinterpretation of LEP searches [37] are also shown for comparison. The excluded region is indicated by the shading.
TABLE VIII. Expected and observed 95\% CL upper limits on the coupling $\lambda$ between unparticles and the SM fields, for values of $d_{\mathcal{U}}$ in the range from 1.01 to 2.20 and a fixed effective cutoff scale $\Lambda_{\mathcal{U}}=10 \mathrm{TeV}$.

\begin{tabular}{lcccc}
\hline \hline & \multicolumn{4}{c}{$\lambda$} \\
\cline { 2 - 5 }$d_{\mathcal{U}}$ & Expected & Expected $-1 \sigma$ & Expected $+1 \sigma$ & Observed \\
\hline 1.01 & 0.045 & 0.038 & 0.053 & 0.044 \\
1.02 & 0.035 & 0.030 & 0.042 & 0.034 \\
1.04 & 0.029 & 0.025 & 0.035 & 0.030 \\
1.06 & 0.028 & 0.024 & 0.033 & 0.028 \\
1.09 & 0.030 & 0.025 & 0.035 & 0.031 \\
1.10 & 0.031 & 0.026 & 0.036 & 0.031 \\
1.30 & 0.085 & 0.072 & 0.100 & 0.087 \\
1.50 & 0.273 & 0.232 & 0.322 & 0.295 \\
1.70 & 0.864 & 0.734 & 1.018 & 0.956 \\
1.90 & 2.86 & 2.43 & 3.37 & 3.22 \\
2.20 & 14.8 & 12.6 & 17.4 & 17.0 \\
\hline \hline
\end{tabular}

TABLE IX. Expected and observed 95\% CL upper limits on the coupling $\lambda$ between unparticles and the SM fields, for values of $d_{\mathcal{U}}$ in the range from 1.01 to 2.20 and a fixed effective cutoff scale $\Lambda_{\mathcal{U}}=100 \mathrm{TeV}$.

\begin{tabular}{lcccc}
\hline \hline & \multicolumn{4}{c}{$\lambda$} \\
\cline { 2 - 5 }$d_{\mathcal{U}}$ & Expected & Expected $-1 \sigma$ & Expected $+1 \sigma$ & Observed \\
\hline 1.01 & 0.046 & 0.039 & 0.054 & 0.045 \\
1.02 & 0.037 & 0.031 & 0.044 & 0.035 \\
1.04 & 0.032 & 0.027 & 0.038 & 0.033 \\
1.06 & 0.032 & 0.027 & 0.038 & 0.033 \\
1.09 & 0.037 & 0.031 & 0.043 & 0.038 \\
1.10 & 0.039 & 0.033 & 0.046 & 0.039 \\
1.30 & 0.169 & 0.143 & 0.199 & 0.174 \\
1.50 & 0.864 & 0.734 & 1.018 & 0.933 \\
1.60 & 1.88 & 1.60 & 2.22 & 2.02 \\
1.70 & 4.33 & 3.68 & 5.10 & 4.79 \\
1.90 & 22.7 & 19.3 & 26.8 & 25.6 \\
2.20 & 235 & 199 & 276 & 270 \\
\hline \hline
\end{tabular}

TABLE X. Expected and observed 95\% CL lower limits on the effective cutoff scale $\Lambda_{\mathcal{U}}$ for values of $d_{\mathcal{U}}$ in the range from 1.60 to 2.20 and a fixed coupling $\lambda=1$.

\begin{tabular}{lcccc}
\hline \hline & \multicolumn{4}{c}{$\Lambda_{\mathcal{U}}(\mathrm{TeV})$} \\
\cline { 2 - 5 }$d_{\mathcal{U}}$ & Expected & Expected $-1 \sigma$ & Expected $+1 \sigma$ & Observed \\
\hline 1.50 & 134 & 186 & 96.4 & 115 \\
1.60 & 34.8 & 45.7 & 26.5 & 30.9 \\
1.70 & 12.3 & 15.6 & 9.75 & 10.7 \\
1.80 & 6.08 & 7.45 & 4.95 & 5.25 \\
1.90 & 3.11 & 3.72 & 2.59 & 2.72 \\
2.00 & 2.09 & 2.46 & 1.77 & 1.85 \\
2.20 & 1.06 & 1.21 & 0.92 & 0.94 \\
\hline \hline
\end{tabular}




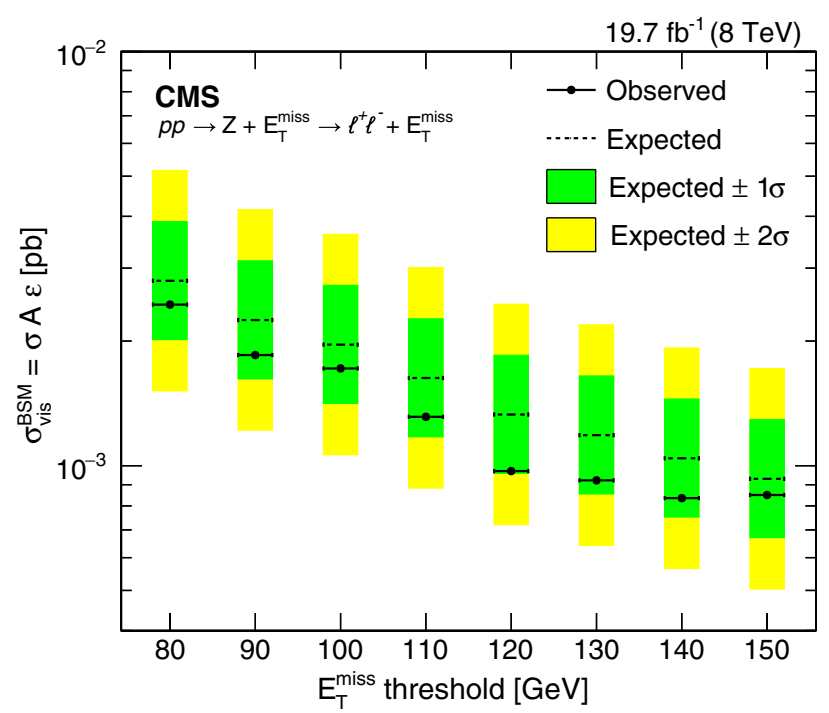

FIG. 10. The model-independent upper limits at 95\% CL on the visible cross section $(\sigma A \epsilon)$ for BSM production of events, as a function of $E_{\mathrm{T}}^{\text {miss }}$ threshold.

In this paper, a particular astrophysical environment with $\left\langle v^{2}\right\rangle=0.24$ is considered, which corresponds to the epoch of the early universe when DM froze out, producing the thermal relic abundance. A $100 \%$ branching fraction of DM annihilating to quarks is assumed. The corresponding truncated limits for D5 and D8 with coupling $\sqrt{g_{q} g_{\chi}}=1$ are also presented with dashed lines in same shade as the untruncated ones. The value required for DM particles to make up the relic abundance is labeled "Thermal relic value" and is shown as a red dotted line. With this constraint on the annihilation rate, we can conclude that Dirac fermion DM is ruled out at 95\% CL for $m_{\chi}<6 \mathrm{GeV}$ in the case of vector coupling and $m_{\chi}<30 \mathrm{GeV}$ in the case of axial-vector coupling. Indirect search results from H.E.S.S [103] and FermiLAT [104] are also shown for comparison. These results have been multiplied by a factor of 2 since they assume Majorana rather than Dirac fermions.

\section{B. Unparticle interpretation}

In the scenario of the unparticle model, the 95\% CL upper limits on the coupling constant $\lambda$ between the unparticle and the SM fields with fixed effective cutoff scales $\Lambda_{\mathcal{U}}=10$ and $100 \mathrm{TeV}$, as functions of the scaling dimension $d_{\mathcal{U}}$, are shown on the left of Fig. 9. The righthand plot of Fig. 9 presents 95\% CL lower limits on the effective cutoff scale $\Lambda_{\mathcal{U}}$ with a fixed coupling $\lambda=1$ and compares the result with the limits obtained from the CMS monojet search [14] and reinterpretation of LEP searches [37]. The search presented in this paper (labeled "monoZ") gives the most stringent limits. Tables VIII and IX show the $95 \%$ CL upper limits on the coupling $\lambda$ between unparticles and the SM fields for values of the scaling dimension $d_{\mathcal{U}}$ in the range from 1.01 to 2.2 and fixed effective cutoff scales of 10 and $100 \mathrm{TeV}$. Lower limits at $95 \% \mathrm{CL}$ on the effective cutoff scale $\Lambda_{\mathcal{U}}$ are given in Table $\mathrm{X}$, for $d_{\mathcal{U}}$ in the range from 1.6 to 2.2 and a fixed coupling $\lambda=1$.

\section{Model-independent limits}

As an alternative to the interpretation of the results in specific models, a single-bin analysis is applied to obtain model-independent expected and observed 95\% CL upper limits on the visible cross section $\sigma_{\text {vis }}^{\mathrm{BSM}}$ for beyond the standard model (BSM) physics processes. The limits as a function of $E_{\mathrm{T}}^{\text {miss }}$ thresholds are shown in Fig. 10. With a $E_{\mathrm{T}}^{\text {miss }}$ threshold of 80 (150) $\mathrm{GeV}$, we exclude the visible cross section $\sigma_{\text {vis }}^{\mathrm{BSM}}>2.5(0.85) \mathrm{fb}$. Table XI shows the total SM background predictions for the numbers of events passing the selection requirements, for different $E_{\mathrm{T}}^{\mathrm{miss}}$ thresholds, compared with the observed numbers of events. The 95\% CL expected and observed upper limits for the contribution of events from BSM sources are also shown.

TABLE XI. Total SM background predictions for the numbers of events passing the selection requirements, for different $E_{\mathrm{T}}^{\mathrm{miss}}$ thresholds, compared with the observed numbers of events. The listed uncertainties include both statistical and systematic components. The $95 \%$ CL observed and expected upper limits for the contribution of events from BSM sources are also shown. The $\pm 1 \sigma$ and $\pm 2 \sigma$ excursions from expected limits are also given.

\begin{tabular}{|c|c|c|c|c|c|c|c|c|}
\hline$E_{\mathrm{T}}^{\text {miss }}(\mathrm{GeV})$ threshold & 80 & 90 & 100 & 110 & 120 & 130 & 140 & 150 \\
\hline Total SM & 263 & 193 & 150 & 117 & 90.5 & 72.5 & 59.2 & 45.1 \\
\hline Total uncertainty & \pm 30 & \pm 24 & \pm 20 & \pm 16 & \pm 13 & \pm 12 & \pm 9.6 & \pm 7.6 \\
\hline Data & 244 & 172 & 141 & 104 & 74 & 61 & 50 & 43 \\
\hline Obs. upper limit & 48.3 & 36.5 & 33.8 & 25.9 & 19.1 & 18.2 & 16.5 & 16.7 \\
\hline Exp. upper limit $+2 \sigma$ & 102 & 81.7 & 71.3 & 59.2 & 48.3 & 43.1 & 37.9 & 33.8 \\
\hline Exp. upper limit $+1 \sigma$ & 76.5 & 61.5 & 53.7 & 44.6 & 36.4 & 32.4 & 28.5 & 25.4 \\
\hline Exp. upper limit & 55.1 & 44.3 & 38.6 & 32.1 & 26.2 & 23.4 & 20.5 & 18.3 \\
\hline Exp. upper limit $-1 \sigma$ & 39.7 & 32.0 & 27.9 & 23.2 & 18.9 & 16.9 & 14.8 & 13.2 \\
\hline Exp. upper limit $-2 \sigma$ & 29.9 & 24.0 & 21.0 & 17.4 & 14.2 & 12.7 & 11.1 & 9.90 \\
\hline
\end{tabular}




\section{SUMMARY}

A search for evidence for particle dark matter and unparticle production at the LHC has been performed in events containing two charged leptons, consistent with the decay of a $Z$ boson, and large missing transverse momentum. The study is based on a data set corresponding to an integrated luminosity of $19.7 \mathrm{fb}^{-1}$ of pp collisions collected by the CMS detector at a center-of-mass energy of $8 \mathrm{TeV}$. The results are consistent with the expected standard model contributions. These results are interpreted in two scenarios for physics beyond the standard model: dark matter and unparticles. Model-independent 95\% confidence level upper limits are also set on contributions to the visible $Z+$ $E_{\mathrm{T}}^{\text {miss }}$ cross section from sources beyond the standard model. Upper limits at $90 \%$ confidence level are set on the DMnucleon scattering cross sections as a function of DM particle mass for both spin-dependent and spin-independent cases. Limits are also set on the DM annihilation rate assuming a branching fraction of $100 \%$ for annihilation to quarks and on the effective cutoff scale. In addition, the most stringent limits to date at $95 \%$ confidence level on the coupling between unparticles and the standard model fields as well as the effective cutoff scale as a function of the unparticle scaling dimension are obtained in this analysis.

\section{ACKNOWLEDGMENTS}

We congratulate our colleagues in the CERN accelerator departments for the excellent performance of the LHC and thank the technical and administrative staffs at CERN and at other CMS institutes for their contributions to the success of the CMS effort. In addition, we gratefully acknowledge the computing centers and personnel of the Worldwide LHC Computing Grid for delivering so effectively the computing infrastructure essential to our analyses. Finally, we acknowledge the enduring support for the construction and operation of the LHC and the CMS detector provided by the following funding agencies: the Austrian Federal Ministry of Science, Research and Economy and the Austrian Science Fund; the Belgian Fonds de la Recherche Scientifique and Fonds voor Wetenschappelijk Onderzoek; the Brazilian Funding Agencies (CNPq, CAPES, FAPERJ, and FAPESP); the Bulgarian Ministry of Education and Science; CERN; the Chinese Academy of Sciences, Ministry of Science and Technology and National Natural Science Foundation of China; the Colombian Funding Agency (COLCIENCIAS); the Croatian Ministry of Science, Education and Sport and the Croatian Science Foundation; the Research Promotion Foundation, Cyprus; the Ministry of Education and Research, Estonian Research Council via IUT23-4 and IUT23-6 and European Regional Development Fund, Estonia; the Academy of Finland, Finnish Ministry of Education and Culture, and Helsinki Institute of Physics; the Institut National de Physique Nucléaire et de Physique des Particules/CNRS and Commissariat à l'Énergie
Atomique et aux Énergies Alternatives/CEA, France; the Bundesministerium für Bildung und Forschung, Deutsche Forschungsgemeinschaft, and Helmholtz-Gemeinschaft Deutscher Forschungszentren, Germany; the General Secretariat for Research and Technology, Greece; the National Scientific Research Foundation and National Innovation Office, Hungary; the Department of Atomic Energy and the Department of Science and Technology, India; the Institute for Studies in Theoretical Physics and Mathematics, Iran; the Science Foundation, Ireland; the Istituto Nazionale di Fisica Nucleare, Italy; the Ministry of Science, ICT and Future Planning, and National Research Foundation (NRF), Republic of Korea; the Lithuanian Academy of Sciences; the Ministry of Education and University of Malaya, Malaysia; the Mexican Funding Agencies (CINVESTAV, CONACYT, SEP, and UASLPFAI); the Ministry of Business, Innovation and Employment, New Zealand; the Pakistan Atomic Energy Commission; the Ministry of Science and Higher Education and the National Science Centre, Poland; the Fundação para a Ciência e a Tecnologia, Portugal; JINR, Dubna, the Ministry of Education and Science of the Russian Federation, the Federal Agency of Atomic Energy of the Russian Federation, Russian Academy of Sciences, and the Russian Foundation for Basic Research; the Ministry of Education, Science and Technological Development of Serbia; the Secretaría de Estado de Investigación, Desarrollo e Innovación and Programa ConsoliderIngenio 2010, Spain; the Swiss Funding Agencies (ETH Board, ETH Zurich, PSI, SNF, UniZH, Canton Zurich, and SER); the Ministry of Science and Technology, Taipei; the Thailand Center of Excellence in Physics, the Institute for the Promotion of Teaching Science and Technology of Thailand, Special Task Force for Activating Research, and the National Science and Technology Development Agency of Thailand; the Scientific and Technical Research Council of Turkey and Turkish Atomic Energy Authority; the National Academy of Sciences of Ukraine and State Fund for Fundamental Researches, Ukraine; the Science and Technology Facilities Council, United Kingdom; the US Department of Energy and the US National Science Foundation. Individuals have received support from the Marie-Curie program and the European Research Council and EPLANET (European Union); the Leventis Foundation; the A. P. Sloan Foundation; the Alexander von Humboldt Foundation; the Belgian Federal Science Policy Office; the Fonds pour la Formation à la Recherche dans l'Industrie et dans l'Agriculture (FRIA-Belgium); the Agentschap voor Innovatie door Wetenschap en Technologie (IWT-Belgium); the Ministry of Education, Youth and Sports (MEYS) of the Czech Republic; the Council of Science and Industrial Research, India; the HOMING PLUS program of the Foundation for Polish Science, cofinanced by the European Union, Regional Development Fund; the OPUS program of the National 
Science Center (Poland); the Compagnia di San Paolo (Torino); MIUR Project No. 20108T4XTM (Italy); the Thalis and Aristeia programs cofinanced by EU-ESF and the Greek NSRF; the National Priorities Research Program by Qatar National Research Fund; the Rachadapisek Sompot Fund for Postdoctoral Fellowship, Chulalongkorn University (Thailand); and the Welch Foundation, Contract No. C-1845.
[1] R. W. Tucker and C. Wang, Dark matter gravitational interactions, Classical Quantum Gravity 15, 933 (1998).

[2] M. Lopez-Corredoira, J. E. Beckman, and E. Casuso, High-velocity clouds as dark matter in the local group, Astron. Astrophys. 351, 920 (1999).

[3] D. Clowe, M. Bradač, A. H. Gonzalez, M. Markevitch, S. W. Randall, C. Jones, and D. Zaritsky, A direct empirical proof of the existence of dark matter, Astrophys. J. 648, L109 (2006).

[4] S. Archambault et al. (PICASSO Collaboration), Constraints on low-mass WIMP interactions on ${ }^{19} \mathrm{~F}$ from PICASSO, Phys. Lett. B 711, 153 (2012).

[5] M. Felizardo et al. (SIMPLE Collaboration), Final Analysis and Results of the Phase II SIMPLE Dark matter Search, Phys. Rev. Lett. 108, 201302 (2012).

[6] E. Behnke et al. (COUPP Collaboration), First dark matter search results from a 4-kg CF 3 I bubble chamber operated in a deep underground site, Phys. Rev. D 86, 052001 (2012).

[7] R. Abbasi et al. (IceCube Collaboration), Multi-year search for dark matter annihilations in the Sun with the AMANDAII and IceCube detectors, Phys. Rev. D 85, 042002 (2012).

[8] R. Agnese et al. (SuperCDMS Collaboration), Search for Low-Mass Weakly Interacting Massive Particles using Voltage-Assisted Calorimetric Ionization Detection in the SuperCDMS Experiment, Phys. Rev. Lett. 112, 041302 (2014).

[9] P. Cushman et al., Snowmass CF1 summary: WIMP dark matter direct detection, arXiv:1310.8327.

[10] R. Agnese et al. (SuperCDMS Collaboration), Search for Low-Mass WIMPs with SuperCDMS, Phys. Rev. Lett. 112, 241302 (2014).

[11] D. S. Akerib et al. (LUX Collaboration), First Results from the LUX Dark Matter Experiment at the Sanford Underground Research Facility, Phys. Rev. Lett. 112, 091303 (2014).

[12] M. Ackermann et al. (Fermi-LAT Collaboration), Constraining Dark Matter Models from a Combined Analysis of Milky Way Satellites with the Fermi Large Area Telescope, Phys. Rev. Lett. 107, 241302 (2011).

[13] J. Buckley et al., Cosmic frontier indirect dark matter detection working group summary, arXiv:1310.7040.

[14] CMS Collaboration, Search for dark matter, extra dimensions, and unparticles in monojet events in proton-proton collisions at $\sqrt{s}=8 \mathrm{TeV}$, Eur. Phys. J. C 75, 235 (2015).

[15] ATLAS Collaboration, Search for new phenomena in final states with an energetic jet and large missing transverse momentum in pp collisions at $\sqrt{s}=8 \mathrm{TeV}$ with the ATLAS detector, Eur. Phys. J. C 75, 299 (2015).
[16] CMS Collaboration, Search for new phenomena in monophoton final states in proton-proton collisions at $\sqrt{s}=8 \mathrm{TeV}$, Phys. Lett. B 755, 102 (2016).

[17] ATLAS Collaboration, Search for new phenomena in events with a photon and missing transverse momentum in pp collisions at $\sqrt{s}=8 \mathrm{TeV}$ with the ATLAS detector, Phys. Rev. D 91, 012008 (2015).

[18] CMS Collaboration, Search for physics beyond the standard model in final states with a lepton and missing transverse energy in proton-proton collisions at $\sqrt{s}=8 \mathrm{TeV}$, Phys. Rev. D 91, 092005 (2015).

[19] ATLAS Collaboration, Search for dark matter in events with heavy quarks and missing transverse momentum in pp collisions with the ATLAS detector, Eur. Phys. J. C 75, 92 (2015).

[20] ATLAS Collaboration, Search for Dark Matter in Events with a Hadronically Decaying W or Z Boson and Missing Transverse Momentum in pp Collisions at $\sqrt{s}=8 \mathrm{TeV}$ with the ATLAS Detector, Phys. Rev. Lett. 112, 041802 (2014).

[21] ATLAS Collaboration, Search for new particles in events with one lepton and missing transverse momentum in $\mathrm{pp}$ collisions at $\sqrt{s}=8 \mathrm{TeV}$ with the ATLAS detector, J. High Energy Phys. 09 (2014) 037.

[22] ATLAS Collaboration, Search for invisible particles produced in association with single-top-quarks in protonproton collisions at $\sqrt{s}=8 \mathrm{TeV}$ with the ATLAS detector, Eur. Phys. J. C 75, 79 (2015).

[23] ATLAS Collaboration, Search for Dark Matter in Events with Missing Transverse Momentum and a Higgs Boson Decaying to Two Photons in pp Collisions at $\sqrt{s}=8 \mathrm{TeV}$ with the ATLAS Detector, Phys. Rev. Lett. 115, 131801 (2015).

[24] CMS Collaboration, Search for Monotop Signatures in Proton-Proton Collisions at $\sqrt{s}=8 \mathrm{TeV}$, Phys. Rev. Lett. 114, 101801 (2015).

[25] CMS Collaboration, Search for the production of dark matter in association with top-quark pairs in the singlelepton final state in proton-proton collisions at $\sqrt{s}=$ 8 TeV, J. High Energy Phys. 06 (2015) 121.

[26] ATLAS Collaboration, Search for dark matter in events with a $\mathrm{Z}$ boson and missing transverse momentum in $\mathrm{pp}$ collisions at $\sqrt{s}=8 \mathrm{TeV}$ with the ATLAS detector, Phys. Rev. D 90, 012004 (2014).

[27] L. M. Carpenter, A. Nelson, C. Shimmin, T. M. P. Tait, and D. Whiteson, Collider searches for dark matter in events with a $\mathrm{Z}$ boson and missing energy, Phys. Rev. D 87, 074005 (2013).

[28] J. Goodman, M. Ibe, A. Rajaraman, W. Shepherd, T. M. P. Tait, and H.-B. Yu, Constraints on dark matter from colliders, Phys. Rev. D 82, 116010 (2010). 
[29] H. Georgi, Unparticle Physics, Phys. Rev. Lett. 98, 221601 (2007).

[30] H. Georgi, Another odd thing about unparticle physics, Phys. Lett. B 650, 275 (2007).

[31] K. Cheung, W.-Y. Keung, and T.-C. Yuan, Collider Signals of Unparticle Physics, Phys. Rev. Lett. 99, 051803 (2007).

[32] K. Cheung, W.-Y. Keung, and T.-C. Yuan, Collider phenomenology of unparticle physics, Phys. Rev. D 76, 055003 (2007).

[33] Z. Kang, Upgrading sterile neutrion dark matter to FImP using scale invariance, Eur. Phys. J. C 75, 471 (2015).

[34] M. Rinaldi, G. Cognola, L. Vanzo, and S. Zerbini, Inflation in scale-invariant theories of gravity, Phys. Rev. D 91, 123527 (2015).

[35] H. Cheng, The Possible Existence of Weyl's Vector Meson, Phys. Rev. Lett. 61, 2182 (1988).

[36] T. Banks and A. Zaks, On the phase structure of vector-like gauge theories with massless fermions, Nucl. Phys. B196, 189 (1982).

[37] S. Kathrein, S. Knapen, and M. J. Strassler, Bounds from LEP on unparticle interactions with electroweak bosons, Phys. Rev. D 84, 015010 (2011).

[38] CMS Collaboration, CMS Physics Analysis Summary CMS-PAS-LUM-13-001， 2013; http://cdsweb.cern.ch/ record/1598864.

[39] CMS Collaboration, The CMS experiment at the CERN LHC, J. Instrum. 3, S08004 (2008).

[40] CMS Collaboration, Performance of electron reconstruction and selection with the CMS detector in proton-proton collisions at $\sqrt{s}=8 \mathrm{TeV}$, J. Instrum. 10, P06005 (2015).

[41] CMS Collaboration, Performance of CMS muon reconstruction in pp collision events at $\sqrt{s}=7 \mathrm{TeV}$, J. Instrum. 7, P10002 (2012).

[42] J. Alwall, R. Frederix, S. Frixione, V. Hirschi, F. Maltoni, O. Mattelaer, H. S. Shao, T. Stelzer, P. Torrielli, and M. Zaro, The automated computation of tree-level and nextto-leading order differential cross sections, and their matching to parton shower simulations.

[43] T. Sjöstrand, S. Mrenna, and P. Skands, PYTHIA 6.4 physics and manual, J. High Energy Phys. 05 (2006) 026.

[44] J. Pumplin, D. R. Stump, J. Huston, H. L. Lai, P. M. Nadolsky, and W. K. Tung, New generation of parton distributions with uncertainties from global QCD analysis, J. High Energy Phys. 07 (2002) 012.

[45] R. Field, Hadron collider physics. Proceedings, 22nd Conference, HCP 2010, Toronto, Canada, 2010 (unpublished).

[46] T. Sjöstrand, S. Mrenna, and P.Z. Skands, A brief introduction to PYTHIA 8.1, Comput. Phys. Commun. 178, 852 (2008).

[47] S. Ask, Simulation of $Z$ plus graviton/unparticle production at the LHC, Eur. Phys. J. C 60, 509 (2009).

[48] S. Ask, I. V. Akin, L. Benucci, A. De Roeck, M. Goebel, and J. Haller, Real emission and virtual exchange of gravitons and unparticles in PYTHIA8, Comput. Phys. Commun. 181, 1593 (2010).

[49] R. Corke and T. Sjöstrand, Interleaved parton showers and tuning prospects, J. High Energy Phys. 03 (2011) 032.

[50] P. Nason, A new method for combining NLO QCD with shower Monte Carlo algorithms, J. High Energy Phys. 11 (2004) 040.
[51] S. Frixione, P. Nason, and C. Oleari, Matching NLO QCD computations with Parton Shower simulations: The POWHEG method, J. High Energy Phys. 11 (2007) 070.

[52] S. Alioli, P. Nason, C. Oleari, and E. Re, A general framework for implementing NLO calculations in shower Monte Carlo programs: the POWHEG BOX, J. High Energy Phys. 06 (2010) 043.

[53] E. Re, Single-top $W t$-channel production matched with parton showers using the POWHEG method, Eur. Phys. J. C 71, 1547 (2011).

[54] S. Alioli, S.-O. Moch, and P. Uwer, Hadronic top-quark pair-production with one jet and parton showering, J. High Energy Phys. 01 (2012) 137.

[55] J. Alwall, P. Demin, S. de Vischer, R. Frederix, M. Herquet, F. Maltoni, T. Plehn, D. L. Rainwater, and T. Stelzer, MadGraph/MadEvent v4: the new web generation, J. High Energy Phys. 09 (2007) 028.

[56] H.-L. Lai, J. Huston, Z. Li, P. Nadolsky, J. Pumplin, D. Stump, and C.-P. Yuan, Uncertainty induced by QCD coupling in the CTEQ global analysis of parton distributions, Phys. Rev. D 82, 054021 (2010).

[57] H.-L. Lai, M. Guzzi, J. Huston, Z. Li, P. M. Nadolsky, J. Pumplin, and C.-P. Yuan, New parton distributions for collider physics, Phys. Rev. D 82, 074024 (2010).

[58] S. Agostinelli et al. (GEANT4), GEANT4-A simulation toolkit, Nucl. Instrum. Methods Phys. Res., Sect. A 506, 250 (2003).

[59] CMS Collaboration, CMS Physics Analysis Summary CMS-PAS-PFT-09-001， 2009; http://cdsweb.cern.ch/ record/1194487.

[60] CMS Collaboration, CMS Physics Analysis Summary CMS-PAS-PFT-10-001， 2010; http://cdsweb.cern.ch/ record/1247373.

[61] M. Cacciari and G. P. Salam, Pileup subtraction using jet areas, Phys. Lett. B 659, 119 (2008).

[62] M. Cacciari, G.P. Salam, and G. Soyez, The anti- $k_{t}$ jet clustering algorithm, J. High Energy Phys. 04 (2008) 063.

[63] M. Cacciari, G. P. Salam, and G. Soyez, FastJet user manual, Eur. Phys. J. C 72, 1896 (2012).

[64] M. Cacciari and G. P. Salam, Dispelling the $N^{3}$ myth for the $k_{t}$ jet-finder, Phys. Lett. B 641, 57 (2006).

[65] CMS Collaboration, Determination of jet energy calibration and transverse momentum resolution in CMS, J. Instrum. 6, P11002 (2011).

[66] CMS Collaboration, Identification of b-quark jets with the CMS experiment, J. Instrum. 8, P04013 (2013).

[67] CMS Collaboration, CMS Physics Analysis Summary CMS-PAS-BTV-13-001，2013; http://cds.cern.ch/record/ 1581306.

[68] J.M. Campbell and R. K. Ellis, MCFM for the Tevatron and the LHC, Nucl. Phys. B, Proc. Suppl. 205-206, 10 (2010).

[69] CMS Collaboration, Measurement of the inclusive $W$ and $Z$ production cross sections in pp collisions at $\sqrt{s}=7 \mathrm{TeV}$, J. High Energy Phys. 10 (2011) 132.

[70] S. Alekhin et al., The PDF4LHC working group interim report, arXiv:1101.0536.

[71] M. Botje, J. Butterworth, A. Cooper-Sarkar, A. de Roeck, J. Feltesse, S. Forte, A. Glazov, J. Huston, R. McNulty, T. Sjöstrand, and R.S. Thorne, The PDF4LHC working group interim recommendations, arXiv:1101.0538. 
[72] R. D. Ball, V. Bertone, S. Carrazza, C. S. Deans, L. Del Debbio, S. Forte, A. Guffanti, N. P. Hartland, J. I. Latorre, J. Rojo, and M. Ubiali (NNPDF Collaboration), Parton distributions with LHC data, Nucl. Phys. B867, 244 (2013).

[73] P. M. Nadolsky, H.-L. Lai, Q.-H. Cao, J. Huston, J. Pumplin, D. Stump, W.-K. Tung, and C.-P. Yuan, Implications of CTEQ global analysis for collider observables, Phys. Rev. D 78, 013004 (2008).

[74] A. D. Martin, W. J. Stirling, R. S. Thorne, and G. Watt, Parton distributions for the LHC, Eur. Phys. J. C 63, 189 (2009).

[75] P. Nason and G. Zanderighi, $W^{+} W^{-}, W Z$ and $Z Z$ production in the POWHEG-BOX-V2, Eur. Phys. J. C 74, 2702 (2014).

[76] T. Gleisberg, S. Hoeche, F. Krauss, M. Schonherr, S. Schumann, F. Siegert, and J. Winter, Event generation with SHERPA 1.1, J. High Energy Phys. 02 (2009) 007.

[77] A. L. Read, Presentation of search results: The $C L_{s}$ technique, J. Phys. G 28, 2693 (2002).

[78] T. Junk, Confidence level computation for combining searches with small statistics, Nucl. Instrum. Methods Phys. Res., Sect. A 434, 435 (1999).

[79] G. Cowan, K. Cranmer, E. Gross, and O. Vitells, Asymptotic formulae for likelihood-based tests of new physics, Eur. Phys. J. C 71, 1554 (2011); 73, 2501 (2013).

[80] ATLAS and CMS Collaborations and LHC Higgs Combination Group, Tech. Rep. ATL-PHYS-PUB-2011-11, CMS NOTE 2011/005, 2011.

[81] P. A. R. Ade et al. (Planck Collaboration), Planck 2013 results. XVI. Cosmological parameters, Astron. Astrophys. 571, A16 (2014).

[82] M. Backović, K. Kong, and M. McCaskey, MadDM v.1.0: Computation of dark matter relic abundance using MadGraph5, Phys. Dark Univ. 5-6, 18 (2014).

[83] Y. Bai, P. J. Fox, and R. Harnik, The Tevatron at the frontier of dark matter direct detection, J. High Energy Phys. 12 (2010) 048.

[84] A. Friedland, M. L. Graesser, I. M. Shoemaker, and L. Vecchi, Probing nonstandard standard model backgrounds with LHC monojets, Phys. Lett. B 714, 267 (2012).

[85] O. Buchmueller, M. J. Dolan, and C. McCabe, Beyond effective field theory for dark matter searches at the LHC, J. High Energy Phys. 01 (2014) 025.

[86] P. J. Fox, R. Harnik, J. Kopp, and Y. Tsai, Missing energy signatures of dark matter at the LHC, Phys. Rev. D 85, 056011 (2012).

[87] J. Goodman and W. Shepherd, LHC bounds on UVcomplete models of dark matter, arXiv:1111.2359.

[88] I. M. Shoemaker and L. Vecchi, Unitarity and monojet bounds on models for DAMA, CoGeNT, and CRESST-II, Phys. Rev. D 86, 015023 (2012).
[89] G. Busoni, A. De Simone, E. Morgante, and A. Riotto, On the validity of the effective field theory for dark matter searches at the LHC, Phys. Lett. B 728, 412 (2014).

[90] G. Busoni, A. De Simone, J. Gramling, E. Morgante, and A. Riotto, On the validity of the effective field theory for dark matter searches at the LHC, part II: complete analysis for the s-channel, J. Cosmol. Astropart. Phys. 06 (2014) 060.

[91] G. Busoni, A. De Simone, T. Jacques, E. Morgante, and A. Riotto, On the Validity of the Effective Field Theory for Dark Matter Searches at the LHC Part III: Analysis for the t-channel, J. Cosmol. Astropart. Phys. 09 (2014) 022.

[92] S. Malik et al., Interplay and characterization of dark matter searches at colliders and in direct detection experiments, Phys. Dark Univ. 9-10, 51 (2015).

[93] J. Abdallah et al., Simplified models for dark matter searches at the LHC, Phys. Dark Univ. 9-10, 8 (2015).

[94] D. Abercrombie et al., Dark matter benchmark models for early LHC Run-2 searches: Report of the ATLAS/CMS dark matter forum, arXiv:1507.00966.

[95] G. Belanger, F. Boudjema, A. Pukhov, and A. Semenov, Dark matter direct detection rate in a generic model with micrOMEGAs 2.2, Comput. Phys. Commun. 180, 747 (2009).

[96] CMS Collaboration, Search for invisible decays of Higgs bosons in the vector boson fusion and associated $\mathrm{ZH}$ production modes, Eur. Phys. J. C 74, 2980 (2014).

[97] A. Djouadi, O. Lebedev, Y. Mambrini, and J. Quevillon, Implications of LHC searches for Higgs-portal dark matter, Phys. Lett. B 709, 65 (2012).

[98] A. Djouadi, A. Falkowski, Y. Mambrini, and J. Quevillon, Direct detection of Higgs-portal dark matter at the LHC, Eur. Phys. J. C 73, 2455 (2013).

[99] R. D. Young and A. W. Thomas, Octet baryon masses and sigma terms from an SU(3) chiral extrapolation, Phys. Rev. D 81, 014503 (2010).

[100] D. Toussaint and W. Freeman (MILC Collaboration), The Strange Quark Condensate in the Nucleon in $2+1$ Flavor QCD, Phys. Rev. Lett. 103, 122002 (2009).

[101] C. Amole et al. (PICO Collaboration), Dark Matter Search Results from the PICO-2L $\mathrm{C}_{3} \mathrm{~F}_{8}$ Bubble Chamber, Phys. Rev. Lett. 114, 231302 (2015).

[102] E. Aprile et al. (XENON100 Collaboration), Limits on Spin-Dependent WIMP-Nucleon Cross Sections from 225 Live Days of XENON100 Data, Phys. Rev. Lett. 111, 021301 (2013).

[103] A. Abramowski et al. (HESS Collaboration), Constraints on an Annihilation Signal from a Core of Constant Dark Matter Density around the Milky Way Center with H.E.S.S., Phys. Rev. Lett. 114, 081301 (2015).

[104] M. Ackermann et al. (Fermi-LAT Collaboration), Dark matter constraints from observations of 25 Milky Way satellite galaxies with the Fermi Large Area telescope, Phys. Rev. D 89, 042001 (2014).

V. Khachatryan, ${ }^{1}$ A. M. Sirunyan, ${ }^{1}$ A. Tumasyan, ${ }^{1}$ W. Adam, ${ }^{2}$ E. Asilar, ${ }^{2}$ T. Bergauer, ${ }^{2}$ J. Brandstetter, ${ }^{2}$ E. Brondolin, ${ }^{2}$ M. Dragicevic, ${ }^{2}$ J. Erö, ${ }^{2}$ M. Flechl, ${ }^{2}$ M. Friedl, ${ }^{2}$ R. Frühwirth, ${ }^{2, b}$ V. M. Ghete, ${ }^{2}$ C. Hartl, ${ }^{2}$ N. Hörmann, ${ }^{2}$ J. Hrubec, ${ }^{2}$ M. Jeitler, ${ }^{2, b}$ V. Knünz, ${ }^{2}$ A. König, ${ }^{2}$ M. Krammer, ${ }^{2, b}$ I. Krätschmer, ${ }^{2}$ D. Liko, ${ }^{2}$ T. Matsushita, ${ }^{2}$ I. Mikulec, ${ }^{2}$ D. Rabady, ${ }^{2, c}$ 
B. Rahbaran, ${ }^{2}$ H. Rohringer, ${ }^{2}$ J. Schieck, ${ }^{2, b}$ R. Schöfbeck, ${ }^{2}$ J. Strauss, ${ }^{2}$ W. Treberer-Treberspurg, ${ }^{2}$ W. Waltenberger, ${ }^{2}$ C.-E. Wulz, ${ }^{2, b}$ V. Mossolov, ${ }^{3}$ N. Shumeiko, ${ }^{3}$ J. Suarez Gonzalez, ${ }^{3}$ S. Alderweireldt, ${ }^{4}$ T. Cornelis, ${ }^{4}$ E. A. De Wolf, ${ }^{4}$ X. Janssen, ${ }^{4}$ A. Knutsson, ${ }^{4}$ J. Lauwers, ${ }^{4}$ S. Luyckx,${ }^{4}$ M. Van De Klundert, ${ }^{4}$ H. Van Haevermaet, ${ }^{4}$ P. Van Mechelen, ${ }^{4}$ N. Van Remortel, ${ }^{4}$ A. Van Spilbeeck, ${ }^{4}$ S. Abu Zeid, ${ }^{5}$ F. Blekman, ${ }^{5}$ J. D’Hondt, ${ }^{5}$ N. Daci, ${ }^{5}$ I. De Bruyn,,${ }^{5}$ K. Deroover, ${ }^{5}$ N. Heracleous, ${ }^{5}$ J. Keaveney, ${ }^{5}$ S. Lowette, ${ }^{5}$ L. Moreels, ${ }^{5}$ A. Olbrechts, ${ }^{5}$ Q. Python, ${ }^{5}$ D. Strom, ${ }^{5}$ S. Tavernier, ${ }^{5}$ W. Van Doninck, ${ }^{5}$ P. Van Mulders, ${ }^{5}$ G. P. Van Onsem, ${ }^{5}$ I. Van Parijs, ${ }^{5}$ P. Barria, ${ }^{6}$ H. Brun, ${ }^{6}$ C. Caillol, ${ }^{6}$ B. Clerbaux, ${ }^{6}$ G. De Lentdecker, ${ }^{6}$ G. Fasanella, ${ }^{6}$ L. Favart, ${ }^{6}$ A. Grebenyuk, ${ }^{6}$ G. Karapostoli, ${ }^{6}$ T. Lenzi, ${ }^{6}$ A. Léonard, ${ }^{6}$ T. Maerschalk, ${ }^{6}$ A. Marinov, ${ }^{6}$ L. Perniè, ${ }^{6}$ A. Randle-conde, ${ }^{6}$ T. Seva, ${ }^{6}$ C. Vander Velde, ${ }^{6}$ P. Vanlaer, ${ }^{6}$ R. Yonamine, ${ }^{6}$ F. Zenoni, ${ }^{6}$ F. Zhang, ${ }^{6, d}$

K. Beernaert, ${ }^{7}$ L. Benucci, ${ }^{7}$ A. Cimmino, ${ }^{7}$ S. Crucy, ${ }^{7}$ D. Dobur, ${ }^{7}$ A. Fagot, ${ }^{7}$ G. Garcia, ${ }^{7}$ M. Gul, ${ }^{7}$ J. Mccartin, ${ }^{7}$ A. A. Ocampo Rios, ${ }^{7}$ D. Poyraz, ${ }^{7}$ D. Ryckbosch, ${ }^{7}$ S. Salva, ${ }^{7}$ M. Sigamani, ${ }^{7}$ M. Tytgat, ${ }^{7}$ W. Van Driessche, ${ }^{7}$ E. Yazgan, ${ }^{7}$ N. Zaganidis, ${ }^{7}$ S. Basegmez,${ }^{8}$ C. Beluffi,,${ }^{8, e}$ O. Bondu, ${ }^{8}$ S. Brochet, ${ }^{8}$ G. Bruno, ${ }^{8}$ A. Caudron, ${ }^{8}$ L. Ceard, ${ }^{8}$ G. G. Da Silveira, ${ }^{8}$ C. Delaere, ${ }^{8}$ D. Favart, ${ }^{8}$ L. Forthomme, ${ }^{8}$ A. Giammanco, ${ }^{8, f}$ J. Hollar, ${ }^{8}$ A. Jafari, ${ }^{8}$ P. Jez, ${ }^{8}$ M. Komm, ${ }^{8}$ V. Lemaitre, ${ }^{8}$ A. Mertens, ${ }^{8}$ M. Musich, ${ }^{8}$ C. Nuttens,${ }^{8}$ L. Perrini, ${ }^{8}$ A. Pin, ${ }^{8}$ K. Piotrzkowski, ${ }^{8}$ A. Popov,${ }^{8, g}$ L. Quertenmont, ${ }^{8}$ M. Selvaggi, ${ }^{8}$

M. Vidal Marono, ${ }^{8}$ N. Beliy, ${ }^{9}$ G. H. Hammad, ${ }^{9}$ W. L. Aldá Júnior, ${ }^{10}$ F. L. Alves, ${ }^{10}$ G. A. Alves, ${ }^{10}$ L. Brito, ${ }^{10}$ M. Correa Martins Junior, ${ }^{10}$ M. Hamer, ${ }^{10}$ C. Hensel, ${ }^{10}$ C. Mora Herrera, ${ }^{10}$ A. Moraes, ${ }^{10}$ M. E. Pol, ${ }^{10}$ P. Rebello Teles,${ }^{10}$ E. Belchior Batista Das Chagas, ${ }^{11}$ W. Carvalho, ${ }^{11}$ J. Chinellato, ${ }^{11, h}$ A. Custódio, ${ }^{11}$ E. M. Da Costa, ${ }^{11}$ D. De Jesus Damiao, ${ }^{11}$ C. De Oliveira Martins, ${ }^{11}$ S. Fonseca De Souza,${ }^{11}$ L. M. Huertas Guativa,${ }^{11}$ H. Malbouisson, ${ }^{11}$ D. Matos Figueiredo, ${ }^{11}$

L. Mundim, ${ }^{11}$ H. Nogima, ${ }^{11}$ W. L. Prado Da Silva, ${ }^{11}$ A. Santoro, ${ }^{11}$ A. Sznajder, ${ }^{11}$ E. J. Tonelli Manganote, ${ }^{11, h}$

A. Vilela Pereira, ${ }^{11}$ S. Ahuja, ${ }^{12 a}$ C. A. Bernardes, ${ }^{12 b}$ A. De Souza Santos, ${ }^{12 b}$ S. Dogra, ${ }^{12 a}$ T. R. Fernandez Perez Tomei, ${ }^{12 a}$ E. M. Gregores, ${ }^{12 b}$ P. G. Mercadante, ${ }^{12 b}$ C. S. Moon, ${ }^{12 a, i}$ S. F. Novaes, ${ }^{12 a}$ Sandra S. Padula, ${ }^{12 a}$ D. Romero Abad, ${ }^{12 a}$ J. C. Ruiz Vargas, ${ }^{12 a}$ A. Aleksandrov, ${ }^{13}$ R. Hadjiiska, ${ }^{13}$ P. Iaydjiev, ${ }^{13}$ M. Rodozov, ${ }^{13}$ S. Stoykova, ${ }^{13}$ G. Sultanov, ${ }^{13}$ M. Vutova, ${ }^{13}$ A. Dimitrov, ${ }^{14}$ I. Glushkov, ${ }^{14}$ L. Litov, ${ }^{14}$ B. Pavlov, ${ }^{14}$ P. Petkov, ${ }^{14}$ M. Ahmad, ${ }^{15}$ J. G. Bian, ${ }^{15}$ G. M. Chen, ${ }^{15}$ H. S. Chen, ${ }^{15}$ M. Chen, ${ }^{15}$ T. Cheng, ${ }^{15}$ R. Du, ${ }^{15}$ C. H. Jiang, ${ }^{15}$ R. Plestina, ${ }^{15, j}$ F. Romeo, ${ }^{15}$ S. M. Shaheen, ${ }^{15}$ A. Spiezia, ${ }^{15}$ J. Tao, ${ }^{15}$ C. Wang, ${ }^{15}$ Z. Wang, ${ }^{15}$ H. Zhang, ${ }^{15}$ C. Asawatangtrakuldee, ${ }^{16}$ Y. Ban, ${ }^{16}$ Q. Li, ${ }^{16}$ S. Liu, ${ }^{16}$ Y. Mao, ${ }^{16}$ S. J. Qian, ${ }^{16}$ D. Wang, ${ }^{16}$ Z. Xu, ${ }^{16}$ C. Avila, ${ }^{17}$ A. Cabrera, ${ }^{17}$ L. F. Chaparro Sierra, ${ }^{17}$ C. Florez, ${ }^{17}$ J. P. Gomez,${ }^{17}$ B. Gomez Moreno, ${ }^{17}$ J. C. Sanabria, ${ }^{17}$ N. Godinovic, ${ }^{18}$ D. Lelas, ${ }^{18}$ I. Puljak, ${ }^{18}$ P. M. Ribeiro Cipriano, ${ }^{18}$ Z. Antunovic, ${ }^{19}$ M. Kovac, ${ }^{19}$

V. Brigljevic, ${ }^{20}$ K. Kadija, ${ }^{20}$ J. Luetic, ${ }^{20}$ S. Micanovic,${ }^{20}$ L. Sudic,${ }^{20}$ A. Attikis,${ }^{21}$ G. Mavromanolakis, ${ }^{21}$ J. Mousa, ${ }^{21}$ C. Nicolaou, ${ }^{21}$ F. Ptochos, ${ }^{21}$ P. A. Razis, ${ }^{21}$ H. Rykaczewski, ${ }^{21}$ M. Bodlak, ${ }^{22}$ M. Finger, ${ }^{22, k}$ M. Finger Jr., ${ }^{22, k}$ Y. Assran, ${ }^{23,1}$

S. Elgammal, ${ }^{23, m}$ A. Ellithi Kamel,,${ }^{23, n, n}$ M. A. Mahmoud, ${ }^{23,0,0}$ Y. Mohammed, ${ }^{23,0}$ B. Calpas, ${ }^{24}$ M. Kadastik,${ }^{24}$ M. Murumaa, ${ }^{24}$ M. Raidal, ${ }^{24}$ A. Tiko, ${ }^{24}$ C. Veelken, ${ }^{24}$ P. Eerola, ${ }^{25}$ J. Pekkanen, ${ }^{25}$ M. Voutilainen, ${ }^{25}$ J. Härkönen, ${ }^{26}$ V. Karimäki, ${ }^{26}$ R. Kinnunen, ${ }^{26}$ T. Lampén, ${ }^{26}$ K. Lassila-Perini ${ }^{26}$ S. Lehti, ${ }^{26}$ T. Lindén, ${ }^{26}$ P. Luukka, ${ }^{26}$ T. Mäenpää, ${ }^{26}$ T. Peltola, ${ }^{26}$ E. Tuominen, ${ }^{26}$ J. Tuominiemi, ${ }^{26}$ E. Tuovinen, ${ }^{26}$ L. Wendland, ${ }^{26}$ J. Talvitie, ${ }^{27}$ T. Tuuva, ${ }^{27}$ M. Besancon, ${ }^{28}$ F. Couderc,${ }^{28}$ M. Dejardin, ${ }^{28}$ D. Denegri, ${ }^{28}$ B. Fabbro, ${ }^{28}$ J. L. Faure, ${ }^{28}$ C. Favaro, ${ }^{28}$ F. Ferri, ${ }^{28}$ S. Ganjour, ${ }^{28}$ A. Givernaud ${ }^{28}$ P. Gras, ${ }^{28}$ G. Hamel de Monchenault, ${ }^{28}$ P. Jarry, ${ }^{28}$ E. Locci, ${ }^{28}$ M. Machet,${ }^{28}$ J. Malcles, ${ }^{28}$ J. Rander, ${ }^{28}$ A. Rosowsky, ${ }^{28}$ M. Titov, ${ }^{28}$ A. Zghiche, ${ }^{28}$ I. Antropov, ${ }^{29}$ S. Baffioni, ${ }^{29}$ F. Beaudette, ${ }^{29}$ P. Busson, ${ }^{29}$ L. Cadamuro, ${ }^{29}$ E. Chapon, ${ }^{29}$ C. Charlot ${ }^{29}$ T. Dahms, ${ }^{29}$ O. Davignon, ${ }^{29}$ N. Filipovic, ${ }^{29}$ R. Granier de Cassagnac, ${ }^{29}$ M. Jo, ${ }^{29}$ S. Lisniak, ${ }^{29}$ L. Mastrolorenzo, ${ }^{29}$ P. Miné, ${ }^{29}$ I. N. Naranjo, ${ }^{29}$ M. Nguyen, ${ }^{29}$ C. Ochando, ${ }^{29}$ G. Ortona, ${ }^{29}$ P. Paganini, ${ }^{29}$ P. Pigard, ${ }^{29}$ S. Regnard ${ }^{29}$ R. Salerno, ${ }^{29}$ J. B. Sauvan, ${ }^{29}$ Y. Sirois, ${ }^{29}$ T. Strebler,${ }^{29}$ Y. Yilmaz,${ }^{29}$ A. Zabi, ${ }^{29}$ J.-L. Agram, ${ }^{30, p}$ J. Andrea, ${ }^{30}$ A. Aubin, ${ }^{30}$ D. Bloch, ${ }^{30}$ J.-M. Brom, ${ }^{30}$ M. Buttignol, ${ }^{30}$ E. C. Chabert, ${ }^{30}$ N. Chanon, ${ }^{30}$ C. Collard,${ }^{30}$ E. Conte,${ }^{30, p}$ X. Coubez,${ }^{30}$ J.-C. Fontaine, ${ }^{30, p}$ D. Gelé, ${ }^{30}$ U. Goerlach ${ }^{30}$ C. Goetzmann, ${ }^{30}$ A.-C. Le Bihan, ${ }^{30}$ J. A. Merlin, ${ }^{30, c}$ K. Skovpen, ${ }^{30}$ P. Van Hove, ${ }^{30}$ S. Gadrat,${ }^{31}$ S. Beauceron, ${ }^{32}$ C. Bernet, ${ }^{32}$ G. Boudoul, ${ }^{32}$ E. Bouvier, ${ }^{32}$ C. A. Carrillo Montoya, ${ }^{32}$ R. Chierici, ${ }^{32}$ D. Contardo, ${ }^{32}$ B. Courbon, ${ }^{32}$ P. Depasse, ${ }^{32}$ H. El Mamouni, ${ }^{32}$ J. Fan, ${ }^{32}$ J. Fay, ${ }^{32}$ S. Gascon, ${ }^{32}$ M. Gouzevitch, ${ }^{32}$ B. Ille, ${ }^{32}$ F. Lagarde, ${ }^{32}$ I. B. Laktineh, ${ }^{32}$ M. Lethuillier, ${ }^{32}$ L. Mirabito, ${ }^{32}$ A. L. Pequegnot, ${ }^{32}$ S. Perries, ${ }^{32}$ J. D. Ruiz Alvarez, ${ }^{32}$ D. Sabes, ${ }^{32}$ L. Sgandurra, ${ }^{32}$ V. Sordini, ${ }^{32}$ M. Vander Donckt, ${ }^{32}$ P. Verdier, ${ }^{32}$ S. Viret, ${ }^{32}$ T. Toriashvili, ${ }^{33, q}$ Z. Tsamalaidze, ${ }^{34, k}$ C. Autermann, ${ }^{35}$ S. Beranek, ${ }^{35}$ M. Edelhoff, ${ }^{35}$ L. Feld, ${ }^{35}$ A. Heister ${ }^{35}$ M. K. Kiesel, ${ }^{35}$ K. Klein, ${ }^{35}$ M. Lipinski, ${ }^{35}$ A. Ostapchuk,${ }^{35}$ M. Preuten, ${ }^{35}$ F. Raupach, ${ }^{35}$ S. Schael,${ }^{35}$ J. F. Schulte, ${ }^{35}$ T. Verlage, ${ }^{35}$ H. Weber, ${ }^{35}$ B. Wittmer, ${ }^{35}$ V. Zhukov, ${ }^{35,9}$ M. Ata, ${ }^{36}$ M. Brodski, ${ }^{36}$ E. Dietz-Laursonn, ${ }^{36}$ D. Duchardt, ${ }^{36}$ M. Endres, ${ }^{36}$ M. Erdmann, ${ }^{36}$ S. Erdweg, ${ }^{36}$ T. Esch, ${ }^{36}$ R. Fischer ${ }^{36}$ A. Güth, ${ }^{36}$ T. Hebbeker, ${ }^{36}$ C. Heidemann, ${ }^{36}$ K. Hoepfner, ${ }^{36}$ S. Knutzen, ${ }^{36}$ P. Kreuzer ${ }^{36}$ M. Merschmeyer, ${ }^{36}$ A. Meyer, ${ }^{36}$ P. Millet, ${ }^{36}$ M. Olschewski, ${ }^{36}$ K. Padeken, ${ }^{36}$ P. Papacz, ${ }^{36}$ T. Pook, ${ }^{36}$ M. Radziej, ${ }^{36}$ 
H. Reithler, ${ }^{36}$ M. Rieger ${ }^{36}$ F. Scheuch ${ }^{36}$ L. Sonnenschein, ${ }^{36}$ D. Teyssier, ${ }^{36}$ S. Thüer, ${ }^{36}$ V. Cherepanov, ${ }^{37}$ Y. Erdogan, ${ }^{37}$ G. Flügge,${ }^{37}$ H. Geenen, ${ }^{37}$ M. Geisler,${ }^{37}$ F. Hoehle, ${ }^{37}$ B. Kargoll, ${ }^{37}$ T. Kress,${ }^{37}$ Y. Kuessel, ${ }^{37}$ A. Künsken, ${ }^{37}$ J. Lingemann, ${ }^{37}$ A. Nehrkorn, ${ }^{37}$ A. Nowack, ${ }^{37}$ I. M. Nugent, ${ }^{37}$ C. Pistone, ${ }^{37}$ O. Pooth,${ }^{37}$ A. Stahl,${ }^{37}$ M. Aldaya Martin, ${ }^{38}$ I. Asin, ${ }^{38}$ N. Bartosik, ${ }^{38}$ O. Behnke, ${ }^{38}$ U. Behrens,${ }^{38}$ A. J. Bell, ${ }^{38}$ K. Borras, ${ }^{38, r}$ A. Burgmeier, ${ }^{38}$ A. Campbell, ${ }^{38}$ S. Choudhury, ${ }^{38, s}$ F. Costanza ${ }^{38}$ C. Diez Pardos, ${ }^{38}$ G. Dolinska, ${ }^{38}$ S. Dooling, ${ }^{38}$ T. Dorland, ${ }^{38}$ G. Eckerlin, ${ }^{38}$ D. Eckstein, ${ }^{38}$ T. Eichhorn, ${ }^{38}$ G. Flucke, ${ }^{38}$ E. Gallo, ${ }^{38, t}$ J. Garay Garcia, ${ }^{38}$ A. Geiser, ${ }^{38}$ A. Gizhko, ${ }^{38}$ P. Gunnellini, ${ }^{38}$ J. Hauk, ${ }^{38}$ M. Hempel,${ }^{38, u}$ H. Jung, ${ }^{38}$ A. Kalogeropoulos, ${ }^{38}$ O. Karacheban, ${ }^{38, u}$ M. Kasemann, ${ }^{38}$ P. Katsas, ${ }^{38}$ J. Kieseler, ${ }^{38}$ C. Kleinwort, ${ }^{38}$ I. Korol, ${ }^{38}$ W. Lange, ${ }^{38}$

J. Leonard ${ }^{38}$ K. Lipka, ${ }^{38}$ A. Lobanov, ${ }^{38}$ W. Lohmann, ${ }^{38, u}$ R. Mankel, ${ }^{38}$ I. Marfin, ${ }^{38, u}$ I.-A. Melzer-Pellmann, ${ }^{38}$

A. B. Meyer, ${ }^{38}$ G. Mittag, ${ }^{38}$ J. Mnich, ${ }^{38}$ A. Mussgiller, ${ }^{38}$ S. Naumann-Emme, ${ }^{38}$ A. Nayak, ${ }^{38}$ E. Ntomari, ${ }^{38}$ H. Perrey, ${ }^{38}$ D. Pitzl,${ }^{38}$ R. Placakyte, ${ }^{38}$ A. Raspereza, ${ }^{38}$ B. Roland,${ }^{38}$ M. Ö. Sahin, ${ }^{38}$ P. Saxena,${ }^{38}$ T. Schoerner-Sadenius, ${ }^{38}$ M. Schröder,${ }^{38}$ C. Seitz, ${ }^{38}$ S. Spannagel, ${ }^{38}$ K. D. Trippkewitz, ${ }^{38}$ R. Walsh, ${ }^{38}$ C. Wissing, ${ }^{38}$ V. Blobel,${ }^{39}$ M. Centis Vignali, ${ }^{39}$ A. R. Draeger, ${ }^{39}$ J. Erfle, ${ }^{39}$ E. Garutti, ${ }^{39}$ K. Goebel,${ }^{39}$ D. Gonzalez, ${ }^{39}$ M. Görner, ${ }^{39}$ J. Haller, ${ }^{39}$ M. Hoffmann, ${ }^{39}$ R. S. Höing,${ }^{39}$ A. Junkes, ${ }^{39}$ R. Klanner, ${ }^{39}$ R. Kogler, ${ }^{39}$ N. Kovalchuk ${ }^{39}$ T. Lapsien, ${ }^{39}$ T. Lenz, ${ }^{39}$ I. Marchesini, ${ }^{39}$ D. Marconi, ${ }^{39}$ M. Meyer, ${ }^{39}$ D. Nowatschin, ${ }^{39}$ J. Ott,${ }^{39}$ F. Pantaleo, ${ }^{39, c}$ T. Peiffer, ${ }^{39}$ A. Perieanu, ${ }^{39}$ N. Pietsch,${ }^{39}$ J. Poehlsen,,${ }^{39}$ D. Rathjens, ${ }^{39}$ C. Sander, ${ }^{39}$ C. Scharf, ${ }^{39}$ H. Schettler, ${ }^{39}$ P. Schleper, ${ }^{39}$ E. Schlieckau, ${ }^{39}$ A. Schmidt, ${ }^{39}$ J. Schwandt, ${ }^{39}$ V. Sola,${ }^{39}$ H. Stadie, ${ }^{39}$

G. Steinbrück, ${ }^{39}$ H. Tholen, ${ }^{39}$ D. Troendle, ${ }^{39}$ E. Usai ${ }^{39}$ L. Vanelderen, ${ }^{39}$ A. Vanhoefer,${ }^{39}$ B. Vormwald ${ }^{39}$ M. Akbiyik, ${ }^{40}$ C. Barth, ${ }^{40}$ C. Baus, ${ }^{40}$ J. Berger, ${ }^{40}$ C. Böser, ${ }^{40}$ E. Butz,${ }^{40}$ T. Chwalek, ${ }^{40}$ F. Colombo,${ }^{40}$ W. De Boer,${ }^{40}$ A. Descroix, ${ }^{40}$ A. Dierlamm, ${ }^{40}$ S. Fink,${ }^{40}$ F. Frensch,${ }^{40}$ R. Friese, ${ }^{40}$ M. Giffels ${ }^{40}$ A. Gilbert ${ }^{40}$ D. Haitz, ${ }^{40}$ F. Hartmann,${ }^{40, c}$ S. M. Heindl,${ }^{40}$ U. Husemann, ${ }^{40}$ I. Katkov, ${ }^{40, g}$ A. Kornmayer, ${ }^{40, c}$ P. Lobelle Pardo, ${ }^{40}$ B. Maier, ${ }^{40}$ H. Mildner, ${ }^{40}$ M. U. Mozer, ${ }^{40}$ T. Müller, ${ }^{40}$ Th. Müller, ${ }^{40}$ M. Plagge, ${ }^{40}$ G. Quast, ${ }^{40}$ K. Rabbertz, ${ }^{40}$ S. Röcker, ${ }^{40}$ F. Roscher, ${ }^{40}$ G. Sieber, ${ }^{40}$ H. J. Simonis, ${ }^{40}$ F. M. Stober ${ }^{40}$ R. Ulrich, ${ }^{40}$ J. Wagner-Kuhr, ${ }^{40} \mathrm{~S}$. Wayand, ${ }^{40} \mathrm{M}$. Weber, ${ }^{40} \mathrm{~T}$. Weiler, ${ }^{40} \mathrm{C}$. Wöhrmann, ${ }^{40} \mathrm{R}$. Wolf, ${ }^{40} \mathrm{G}$. Anagnostou, ${ }^{41}$ G. Daskalakis, ${ }^{41}$ T. Geralis, ${ }^{41}$ V. A. Giakoumopoulou, ${ }^{41}$ A. Kyriakis, ${ }^{41}$ D. Loukas ${ }^{41}$ A. Psallidas, ${ }^{41}$ I. Topsis-Giotis, ${ }^{41}$ A. Agapitos ${ }^{42}$ S. Kesisoglou, ${ }^{42}$ A. Panagiotou, ${ }^{42}$ N. Saoulidou, ${ }^{42}$ E. Tziaferi, ${ }^{42}$ I. Evangelou, ${ }^{43}$ G. Flouris ${ }^{43}$ C. Foudas,${ }^{43}$ P. Kokkas, ${ }^{43}$ N. Loukas,${ }^{43}$ N. Manthos,${ }^{43}$ I. Papadopoulos,${ }^{43}$ E. Paradas,${ }^{43}$ J. Strologas,${ }^{43}$ G. Bencze,${ }^{44}$ C. Hajdu,${ }^{44}$ A. Hazi,${ }^{44}$ P. Hidas, ${ }^{44}$ D. Horvath, ${ }^{44, v}$ F. Sikler, ${ }^{44}$ V. Veszpremi ${ }^{44}$ G. Vesztergombi, ${ }^{44, w}$ A. J. Zsigmond ${ }^{44}$ N. Beni, ${ }^{45}$ S. Czellar, ${ }^{45}$ J. Karancsi, ${ }^{45, \mathrm{x}}$ J. Molnar, ${ }^{45}$ Z. Szillasi, ${ }^{45, \mathrm{c}}$ M. Bartók, ${ }^{46, y}$ A. Makovec, ${ }^{46}$ P. Raics, ${ }^{46}$ Z. L. Trocsanyi, ${ }^{46}$ B. Ujvari, ${ }^{46}$ P. Mal, ${ }^{47}$ K. Mandal, ${ }^{47}$ D. K. Sahoo, ${ }^{47}$ N. Sahoo, ${ }^{47}$ S. K. Swain, ${ }^{47}$ S. Bansal, ${ }^{48}$ S. B. Beri, ${ }^{48}$ V. Bhatnagar, ${ }^{48}$ R. Chawla,${ }^{48}$ R. Gupta, ${ }^{48}$ U. Bhawandeep, ${ }^{48}$ A. K. Kalsi, ${ }^{48}$ A. Kaur, ${ }^{48}$ M. Kaur ${ }^{48}$ R. Kumar, ${ }^{48}$ A. Mehta, ${ }^{48}$ M. Mittal, ${ }^{48}$ J. B. Singh, ${ }^{48}$ G. Walia, ${ }^{48}$ Ashok Kumar, ${ }^{49}$ A. Bhardwaj, ${ }^{49}$ B. C. Choudhary, ${ }^{49}$ R. B. Garg, ${ }^{49}$ A. Kumar, ${ }^{49}$ S. Malhotra, ${ }^{49}$ M. Naimuddin,${ }^{49}$ N. Nishu, ${ }^{49}$ K. Ranjan, ${ }^{49}$ R. Sharma, ${ }^{49}$ V. Sharma, ${ }^{49}$ S. Bhattacharya,${ }^{50}$ K. Chatterjee, ${ }^{50}$ S. Dey, ${ }^{50}$ S. Dutta,${ }^{50}$ Sa. Jain,${ }^{50}$ N. Majumdar,${ }^{50}$ A. Modak,${ }^{50}$ K. Mondal, ${ }^{50}$ S. Mukherjee, ${ }^{50}$ S. Mukhopadhyay, ${ }^{50}$ A. Roy, ${ }^{50}$ D. Roy, ${ }^{50}$ S. Roy Chowdhury, ${ }^{50}$ S. Sarkar,${ }^{50}$ M. Sharan, ${ }^{50}$ A. Abdulsalam, ${ }^{51}$ R. Chudasama, ${ }^{51}$ D. Dutta, ${ }^{51}$ V. Jha, ${ }^{51}$ V. Kumar, ${ }^{51}$ A. K. Mohanty, ${ }^{51, c}$ L. M. Pant, ${ }^{51}$ P. Shukla, ${ }^{51}$ A. Topkar, ${ }^{51}$ T. Aziz, ${ }^{52}$ S. Banerjee, ${ }^{52}$ S. Bhowmik, ${ }^{52, z}$ R. M. Chatterjee, ${ }^{52}$ R. K. Dewanjee, ${ }^{52}$ S. Dugad ${ }^{52}$ S. Ganguly, ${ }^{52}$ S. Ghosh, ${ }^{52}$ M. Guchait, ${ }^{52}$ A. Gurtu, ${ }^{52, a a}$ G. Kole, ${ }^{52}$ S. Kumar,${ }^{52}$ B. Mahakud, ${ }^{52}$ M. Maity, ${ }^{52, z}$ G. Majumder, ${ }^{52}$ K. Mazumdar, ${ }^{52}$ S. Mitra, ${ }^{52}$ G. B. Mohanty, ${ }^{52}$ B. Parida, ${ }^{52}$ T. Sarkar, ${ }^{52, z}$ N. Sur, ${ }^{52}$ B. Sutar, ${ }^{52}$ N. Wickramage, ${ }^{52, b b}$ S. Chauhan, ${ }^{53}$ S. Dube,${ }^{53}$ K. Kothekar, ${ }^{53}$ S. Sharma, ${ }^{53}$ H. Bakhshiansohi,${ }^{54}$ H. Behnamian, ${ }^{54}$ S. M. Etesami, ${ }^{54, c c}$ A. Fahim, ${ }^{54, d d}$ R. Goldouzian, ${ }^{54}$ M. Khakzad, ${ }^{54}$ M. Mohammadi Najafabadi, ${ }^{54}$ M. Naseri, ${ }^{54}$ S. Paktinat Mehdiabadi, ${ }^{54}$ F. Rezaei Hosseinabadi, ${ }^{54}$ B. Safarzadeh,${ }^{54, \mathrm{ee}}$ M. Zeinali, ${ }^{54}$ M. Felcini, ${ }^{55}$ M. Grunewald,${ }^{55}$ M. Abbrescia,${ }^{56,56 \mathrm{~b}}$ C. Calabria,${ }^{56,56 \mathrm{~b}}$ C. Caputo, ${ }^{56 a, 56 \mathrm{~b}}$ A. Colaleo, ${ }^{56 \mathrm{a}}$ D. Creanza, ${ }^{56 \mathrm{a}, 56 \mathrm{c}}$ L. Cristella ${ }^{56 \mathrm{a}, 56 \mathrm{~b}}$ N. De Filippis,${ }^{56,56 \mathrm{c}}$ M. De Palma, ${ }^{56 a, 56 b}$ L. Fiore, ${ }^{56 a}$ G. Iaselli, ${ }^{56 a, 56 c}$ G. Maggi, ${ }^{56 a, 56 c}$ M. Maggi, ${ }^{56 a}$ G. Miniello, ${ }^{56 a, 56 b}$ S. My, ${ }^{56 a, 56 c}$ S. Nuzzo, ${ }^{56 a, 56 b}$ A. Pompili, ${ }^{56 a, 56 b}$ G. Pugliese,${ }^{56 a, 56 \mathrm{c}}$ R. Radogna,${ }^{56 \mathrm{a}, 56 \mathrm{~b}}$ A. Ranieri, ${ }^{56 \mathrm{a}}$ G. Selvaggi, ${ }^{56 \mathrm{a}, 56 \mathrm{~b}}$ L. Silvestris, ${ }^{56 \mathrm{a}, \mathrm{c}}$ R. Venditti, ${ }^{56 \mathrm{a}, 56 \mathrm{~b}}$ P. Verwilligen, ${ }^{56 a}$ G. Abbiendi, ${ }^{57 a}$ C. Battilana, ${ }^{57 a, c}$ A. C. Benvenuti, ${ }^{57 a}$ D. Bonacorsi, ${ }^{57 a, 57 b}$ S. Braibant-Giacomelli, ${ }^{57 a, 57 b}$ L. Brigliadori, ${ }^{57 a, 57 b}$ R. Campanini, ${ }^{57 a, 57 b}$ P. Capiluppi, ${ }^{57,57 b}$ A. Castro, ${ }^{57 a, 57 b}$ F. R. Cavallo, ${ }^{57 a}$ S. S. Chhibra, ${ }^{57,57 b}$ G. Codispoti, ${ }^{57,57 b}$ M. Cuffiani, ${ }^{57 a, 57 b}$ G. M. Dallavalle, ${ }^{57 a}$ F. Fabbri, ${ }^{57 a}$ A. Fanfani, ${ }^{57 a, 57 b}$ D. Fasanella, ${ }^{57 a, 57 b}$ P. Giacomelli, ${ }^{57 a}$ C. Grandi, ${ }^{57 a}$ L. Guiducci, ${ }^{57 a, 57 b}$ S. Marcellini, ${ }^{57 a}$ G. Masetti, ${ }^{57 a}$ A. Montanari, ${ }^{57 a}$ F. L. Navarria, ${ }^{57 a, 57 b}$ A. Perrotta, ${ }^{57 a}$ A. M. Rossi, ${ }^{57,57 b}$ T. Rovelli, ${ }^{57 a, 57 b}$ G. P. Siroli, ${ }^{57 a, 57 b}$ N. Tosi,${ }^{57 a, 57 b, c}$ R. Travaglini, ${ }^{57 a, 57 b}$ G. Cappello, ${ }^{58 a}$ M. Chiorboli, ${ }^{58 a, 58 b}$ S. Costa, ${ }^{58 a, 58 b}$ A. Di Mattia, ${ }^{58 a}$ F. Giordano, ${ }^{58 a, 58 b}$ R. Potenza ${ }^{58 a, 58 b}$ A. Tricomi,${ }^{58,58 b}$ C. Tuve, ${ }^{58 a, 58 b}$ G. Barbagli, ${ }^{59 a}$ V. Ciulli, ${ }^{59,59 b}$ C. Civinini, ${ }^{59 a}$ R. D’Alessandro, ${ }^{59 a, 59 b}$ E. Focardi, ${ }^{59 a, 59 b}$ S. Gonzi, ${ }^{59 a, 59 b}$ V. Gori, ${ }^{59 a, 59 b}$ P. Lenzi, ${ }^{59 a, 59 b}$ M. Meschini, ${ }^{59 a}$ S. Paoletti, ${ }^{59 a}$ G. Sguazzoni, ${ }^{59 a}$ A. Tropiano, ${ }^{59 a, 59 b}$ L. Viliani, ${ }^{59 a, 59 b, c}$ L. Benussi, ${ }^{60}$ 
S. Bianco, ${ }^{60}$ F. Fabbri, ${ }^{60}$ D. Piccolo ${ }^{60}$ F. Primavera ${ }^{60, c}$ V. Calvelli, ${ }^{61 a, 61 b}$ F. Ferro,${ }^{61 a}$ M. Lo Vetere,,${ }^{61,61 b}$ M. R. Monge, ${ }^{61 a, 61 b}$ E. Robutti, ${ }^{61 a}$ S. Tosi, ${ }^{61 a, 61 b}$ L. Brianza, ${ }^{62 a}$ M. E. Dinardo, ${ }^{62 a, 62 b}$ S. Fiorendi, ${ }^{62 a, 62 b}$ S. Gennai, ${ }^{62 a}$ R. Gerosa, ${ }^{62 a, 62 b}$ A. Ghezzi, ${ }^{62 a, 62 b}$ P. Govoni, ${ }^{62 a, 62 b}$ S. Malvezzi, ${ }^{62 a}$ R. A. Manzoni, ${ }^{62 a, 62 b, c}$ B. Marzocchi, ${ }^{62 a, 62 b}$ D. Menasce, ${ }^{62 a}$ L. Moroni, ${ }^{62 a}$ M. Paganoni, ${ }^{62 a, 62 b}$ D. Pedrini, ${ }^{6 a}$ S. Ragazzi, ${ }^{62 a, 62 b}$ N. Redaelli, ${ }^{62 a}$ T. Tabarelli de Fatis, ${ }^{62 a, 62 b}$ S. Buontempo, ${ }^{63 a}$ N. Cavallo, ${ }^{63 a, 63 c}$ S. Di Guida,${ }^{63 a, 63 d, c}$ M. Esposito, ${ }^{63 a, 63 b}$ F. Fabozzi, ${ }^{63 a, 63 c}$ A. O. M. Iorio, ${ }^{63 a, 63 b}$ G. Lanza, ${ }^{63 a}$ L. Lista, ${ }^{63 a}$ S. Meola ${ }^{63 a, 63 d, c}$ M. Merola,${ }^{63 a}$ P. Paolucci, ${ }^{63 a, c}$ C. Sciacca,${ }^{63 a, 63 b}$ F. Thyssen, ${ }^{63 a}$ P. Azzi ${ }^{64 a, c}$ N. Bacchetta, ${ }^{64 a}$ L. Benato,${ }^{64 a, 64 b}$ D. Bisello, ${ }^{64 a, 64 b}$ A. Boletti, ${ }^{64 a, 64 b}$ A. Branca, ${ }^{64 a, 64 b}$ R. Carlin,${ }^{64 a, 64 b}$ P. Checchia, ${ }^{64 a}$ M. Dall'Osso, ${ }^{64 a, 64 b, c}$ T. Dorigo, ${ }^{64 a}$

U. Dosselli, ${ }^{64 a}$ F. Gasparini, ${ }^{64 a, 64 b}$ U. Gasparini, ${ }^{64 a, 64 b}$ A. Gozzelino, ${ }^{64 a}$ K. Kanishchev, ${ }^{64 a, 64 c}$ S. Lacaprara, ${ }^{64 a}$ M. Margoni ${ }^{64 a, 64 b}$ A. T. Meneguzzo, ${ }^{64 a, 64 b}$ M. Passaseo, ${ }^{64 a}$ J. Pazzini, ${ }^{64,64 b, c}$ N. Pozzobon, ${ }^{64 a, 64 b}$ P. Ronchese, ${ }^{64 a, 64 b}$ F. Simonetto, ${ }^{64 a, 64 b}$ E. Torassa, ${ }^{64 a}$ M. Tosi ${ }^{64 a, 64 b}$ M. Zanetti, ${ }^{64 a}$ P. Zotto, ${ }^{64 a, 64 b}$ A. Zucchetta,${ }^{64 a, 64 b, c}$ G. Zumerle, ${ }^{64 a, 64 b}$ A. Braghieri, ${ }^{65 a}$ A. Magnani, ${ }^{65 a}$ P. Montagna, ${ }^{65 a, 65 b}$ S. P. Ratti, ${ }^{65 a, 65 b}$ V. Re, ${ }^{65 a}$ C. Riccardi, ${ }^{65 a, 65 b}$ P. Salvini, ${ }^{65 a}$ I. Vai, ${ }^{65 a}$ P. Vitulo, ${ }^{65 a, 65 b}$ L. Alunni Solestizi, ${ }^{66 a, 66 b}$ G. M. Bilei, ${ }^{66 \mathrm{a}}$ D. Ciangottini, ${ }^{66 a, 66 b, c}$ L. Fanò, ${ }^{66 a, 66 b}$ P. Lariccia, ${ }^{66 a, 66 b}$ G. Mantovani ${ }^{66 a, 66 b}$ M. Menichelli, ${ }^{66 a}$ A. Saha ${ }^{66 a}$ A. Santocchia,${ }^{66 a, 66 b}$ K. Androsov,${ }^{67 a, f f}$ P. Azzurri, ${ }^{67, c}$ G. Bagliesi,${ }^{67 a}$ J. Bernardini, ${ }^{67 \mathrm{a}}$ T. Boccali, ${ }^{67 \mathrm{a}}$ R. Castaldi, ${ }^{67 \mathrm{a}}$ M. A. Ciocci, ${ }^{67 \mathrm{a} f \mathrm{ff}}$ R. Dell'Orso, ${ }^{67 \mathrm{a}}$ S. Donato, ${ }^{67 \mathrm{a}, 67 \mathrm{c}, \mathrm{c}}$ G. Fedi, ${ }^{67 \mathrm{a}}$ L. Foà, ${ }^{67 \mathrm{a}, 67 \mathrm{c}, \mathrm{a}}$ A. Giassi, ${ }^{67 a}$ M. T. Grippo, ${ }^{67 a, f f}$ F. Ligabue,${ }^{67 a, 67 c}$ T. Lomtadze, ${ }^{67 a}$ L. Martini,${ }^{67 a, 67 b}$ A. Messineo, ${ }^{67 a, 67 b}$ F. Palla, ${ }^{67 a}$ A. Rizzi, ${ }^{67,67 b}$ A. Savoy-Navarro, ${ }^{67 a, g g}$ A. T. Serban, ${ }^{67 a}$ P. Spagnolo, ${ }^{67 a}$ R. Tenchini,${ }^{67 a}$ G. Tonelli, ${ }^{67 a, 67 b}$ A. Venturi, ${ }^{67 a}$ P. G. Verdini ${ }^{67 a}$ L. Barone, ${ }^{68 a, 68 b}$ F. Cavallari, ${ }^{68 a}$ G. D'imperio, ${ }^{68 a, 68 b, c}$ D. Del Re ${ }^{68 a, 68 b, c}$ M. Diemoz, ${ }^{68 a}$ S. Gelli, ${ }^{68 a, 68 b}$ C. Jorda, ${ }^{68 \mathrm{a}}$ E. Longo, ${ }^{68 \mathrm{a}, 68 \mathrm{~b}}$ F. Margaroli, ${ }^{68 \mathrm{a}, 68 \mathrm{~b}}$ P. Meridiani, ${ }^{68 \mathrm{a}}$ G. Organtini, ${ }^{68 \mathrm{a}, 68 \mathrm{~b}}$ R. Paramatti, ${ }^{68 \mathrm{a}}$ F. Preiato, ${ }^{68 a, 68 b}$ S. Rahatlou, ${ }^{68 \mathrm{a}, 68 \mathrm{~b}}$ C. Rovelli, ${ }^{68 \mathrm{a}}$ F. Santanastasio ${ }^{68 \mathrm{a}, 68 \mathrm{~b}}$ P. Traczyk, ${ }^{68 \mathrm{a}, 68 \mathrm{~b}, \mathrm{c}}$ N. Amapane ${ }^{69 \mathrm{a}, 69 \mathrm{~b}}$ R. Arcidiacono, ${ }^{69 \mathrm{a}, 69 \mathrm{c}, \mathrm{c}}$ S. Argiro, ${ }^{69 a, 69 b}$ M. Arneodo, ${ }^{69 a, 69 c}$ R. Bellan, ${ }^{69 a, 69 b}$ C. Biino, ${ }^{69 a}$ N. Cartiglia ${ }^{69 a}$ M. Costa, ${ }^{69 a, 69 b}$ R. Covarelli, ${ }^{69 a, 69 b}$ A. Degano, ${ }^{69 a, 69 b}$ N. Demaria ${ }^{69 a}$ L. Finco, ${ }^{69 a, 69 b, c}$ C. Mariotti, ${ }^{69 a}$ S. Maselli, ${ }^{69 a}$ G. Mazza,${ }^{69 a}$ E. Migliore,${ }^{69,69 b}$ V. Monaco, ${ }^{69 a, 69 b}$ E. Monteil, ${ }^{69 a, 69 b}$ M. M. Obertino, ${ }^{69 a, 69 b}$ L. Pacher, ${ }^{69 a, 69 b}$ N. Pastrone,${ }^{69 a}$ M. Pelliccioni, ${ }^{69 a}$ G. L. Pinna Angioni ${ }^{69 \mathrm{a}, 69 \mathrm{~b}}$ F. Ravera ${ }^{69 \mathrm{a}, 69 \mathrm{~b}}$ A. Romero, ${ }^{69 \mathrm{a}, 69 \mathrm{~b}}$ M. Ruspa,${ }^{69 a, 69 \mathrm{c}}$ R. Sacchi, ${ }^{69 a, 69 \mathrm{~b}}$ A. Solano, ${ }^{69 \mathrm{a}, 69 \mathrm{~b}}$ A. Staiano, ${ }^{69 a}$ S. Belforte, ${ }^{70 a}$ V. Candelise,${ }^{70 a, 70 b, c}$ M. Casarsa ${ }^{70 a}$ F. Cossutti,${ }^{70 a}$ G. Della Ricca, ${ }^{70 a, 70 b}$ B. Gobbo,${ }^{70 a}$ C. La Licata, ${ }^{70 a, 70 b}$ M. Marone, ${ }^{70 a, 70 b}$ A. Schizzi, ${ }^{70 a, 70 b}$ A. Zanetti, ${ }^{70 a}$ A. Kropivnitskaya, ${ }^{71}$ S. K. Nam, ${ }^{71}$ D. H. Kim, ${ }^{72}$ G. N. Kim, ${ }^{72}$ M. S. Kim, ${ }^{72}$ D. J. Kong, ${ }^{72}$ S. Lee, ${ }^{72}$ Y. D. Oh, ${ }^{72}$ A. Sakharov, ${ }^{72}$ D. C. Son, ${ }^{72}$ J. A. Brochero Cifuentes ${ }^{73}$ H. Kim, ${ }^{73}$ T. J. Kim, ${ }^{73}$ S. Song ${ }^{74}$ S. Choi ${ }^{75}$ Y. Go, ${ }^{75}$ D. Gyun, ${ }^{75}$ B. Hong,${ }^{75}$ H. Kim,${ }^{75}$ Y. Kim, ${ }^{75}$ B. Lee ${ }^{75}$ K. Lee, ${ }^{75}$ K. S. Lee, ${ }^{75}$ S. Lee, ${ }^{75}$ S. K. Park, ${ }^{75}$ Y. Roh,${ }^{75}$ H. D. Yoo ${ }^{76}$ M. Choi,${ }^{77}$ H. Kim,${ }^{77}$ J. H. Kim, ${ }^{77}$ J. S. H. Lee, ${ }^{77}$ I. C. Park, ${ }^{77}$ G. Ryu ${ }^{77}$ M. S. Ryu ${ }^{77}$ Y. Choi, ${ }^{78}$ J. Goh, ${ }^{78}$ D. Kim, ${ }^{78}$ E. Kwon, ${ }^{78}$ J. Lee, ${ }^{78}$ I. Yu, ${ }^{78}$ V. Dudenas, ${ }^{79}$ A. Juodagalvis ${ }^{79}$ J. Vaitkus, ${ }^{79}$ I. Ahmed,${ }^{80}$ Z. A. Ibrahim, ${ }^{80}$ J. R. Komaragiri, ${ }^{80}$ M. A. B. Md Ali, ${ }^{80, \text { hh }}$ F. Mohamad Idris, ${ }^{80, \text { ii }}$

W. A. T. Wan Abdullah, ${ }^{80}$ M. N. Yusli, ${ }^{80}$ E. Casimiro Linares ${ }^{81}$ H. Castilla-Valdez,${ }^{81}$ E. De La Cruz-Burelo, ${ }^{81}$

I. Heredia-De La Cruz, ${ }^{81, j j}$ A. Hernandez-Almada, ${ }^{81}$ R. Lopez-Fernandez, ${ }^{81}$ A. Sanchez-Hernandez, ${ }^{81}$ S. Carrillo Moreno, ${ }^{82}$

F. Vazquez Valencia, ${ }^{82}$ I. Pedraza, ${ }^{83}$ H. A. Salazar Ibarguen, ${ }^{83}$ A. Morelos Pineda, ${ }^{84}$ D. Krofcheck, ${ }^{85}$ P. H. Butler, ${ }^{86}$ A. Ahmad, ${ }^{87}$ M. Ahmad, ${ }^{87}$ Q. Hassan, ${ }^{87}$ H. R. Hoorani, ${ }^{87}$ W. A. Khan, ${ }^{87}$ T. Khurshid, ${ }^{87}$ M. Shoaib ${ }^{87}$ H. Bialkowska ${ }^{88}$ M. Bluj ${ }^{88}$ B. Boimska, ${ }^{88}$ T. Frueboes, ${ }^{88}$ M. Górski, ${ }^{88}$ M. Kazana,${ }^{88}$ K. Nawrocki, ${ }^{88}$ K. Romanowska-Rybinska, ${ }^{88}$ M. Szleper, ${ }^{88}$ P. Zalewski, ${ }^{88}$ G. Brona ${ }^{89}$ K. Bunkowski, ${ }^{89}$ A. Byszuk, ${ }^{89, k k}$ K. Doroba ${ }^{89}$ A. Kalinowski,${ }^{89}$ M. Konecki, ${ }^{89}$ J. Krolikowski, ${ }^{89}$ M. Misiura, ${ }^{89}$ M. Olszewski, ${ }^{89}$ M. Walczak, ${ }^{89}$ P. Bargassa ${ }^{90}$ C. Beirão Da Cruz E Silva, ${ }^{90}$ A. Di Francesco, ${ }^{90}$ P. Faccioli, ${ }^{90}$ P. G. Ferreira Parracho, ${ }^{90}$ M. Gallinaro, ${ }^{90}$ N. Leonardo, ${ }^{90}$ L. Lloret Iglesias, ${ }^{90}$ F. Nguyen, ${ }^{90}$ J. Rodrigues Antunes, ${ }^{90}$ J. Seixas, ${ }^{90}$ O. Toldaiev,${ }^{90}$ D. Vadruccio,${ }^{90}$ J. Varela,${ }^{90}$ P. Vischia, ${ }^{90}$ S. Afanasiev, ${ }^{91}$ P. Bunin, ${ }^{91}$ M. Gavrilenko, ${ }^{91}$ I. Golutvin, ${ }^{91}$ I. Gorbunov, ${ }^{91}$ A. Kamenev,${ }^{91}$ V. Karjavin, ${ }^{91}$ V. Konoplyanikov, ${ }^{91}$ A. Lanev, ${ }^{91}$ A. Malakhov, ${ }^{91}$ V. Matveev, ${ }^{91,11, \mathrm{~mm}}$ P. Moisenz, ${ }^{91}$ V. Palichik, ${ }^{91}$ V. Perelygin, ${ }^{91}$ S. Shmatov, ${ }^{91}$ S. Shulha, ${ }^{91}$ N. Skatchkov,${ }^{91}$ V. Smirnov, ${ }^{91}$ A. Zarubin, ${ }^{91}$ V. Golovtsov, ${ }^{92}$ Y. Ivanov, ${ }^{92}$ V. Kim, ${ }^{92, n n}$ E. Kuznetsova, ${ }^{92}$ P. Levchenko, ${ }^{92}$ V. Murzin, ${ }^{92}$ V. Oreshkin, ${ }^{92}$ I. Smirnov, ${ }^{92}$ V. Sulimov, ${ }^{92}$ L. Uvarov, ${ }^{92}$ S. Vavilov, ${ }^{92}$ A. Vorobyev,${ }^{92}$ Yu. Andreev, ${ }^{93}$ A. Dermenev, ${ }^{93}$ S. Gninenko, ${ }^{93}$ N. Golubev, ${ }^{93}$ A. Karneyeu, ${ }^{93}$ M. Kirsanov, ${ }^{93}$ N. Krasnikov,${ }^{93}$ A. Pashenkov,${ }^{93}$ D. Tlisov, ${ }^{93}$ A. Toropin, ${ }^{93}$ V. Epshteyn, ${ }^{94}$ V. Gavrilov, ${ }^{94}$ N. Lychkovskaya, ${ }^{94}$ V. Popov, ${ }^{94}$ I. Pozdnyakov, ${ }^{94}$ G. Safronov, ${ }^{94}$ A. Spiridonov, ${ }^{94}$ E. Vlasov, ${ }^{94}$

A. Zhokin, ${ }^{94}$ A. Bylinkin, ${ }^{95}$ V. Andreev,${ }^{96}$ M. Azarkin, ${ }^{96, \mathrm{~mm}}$ I. Dremin, ${ }^{96, \mathrm{~mm}}$ M. Kirakosyan, ${ }^{96}$ A. Leonidov, ${ }^{96, \mathrm{~mm}}$ G. Mesyats, ${ }^{96}$ S. V. Rusakov, ${ }^{96}$ A. Baskakov, ${ }^{97}$ A. Belyaev, ${ }^{97}$ E. Boos,${ }^{97}$ M. Dubinin,${ }^{97,00}$ L. Dudko, ${ }^{97}$ A. Ershov,${ }^{97}$ A. Gribushin, ${ }^{97}$ V. Klyukhin, ${ }^{97}$ O. Kodolova, ${ }^{97}$ I. Lokhtin, ${ }^{97}$ I. Myagkov, ${ }^{97}$ S. Obraztsov, ${ }^{97}$ S. Petrushanko, ${ }^{97}$ V. Savrin, ${ }^{97}$ A. Snigirev, ${ }^{97}$ I. Azhgirey, ${ }^{98}$ I. Bayshev, ${ }^{98}$ S. Bitioukov, ${ }^{98}$ V. Kachanov, ${ }^{98}$ A. Kalinin, ${ }^{98}$ D. Konstantinov, ${ }^{98}$ V. Krychkine, ${ }^{98}$ 
V. Petrov, ${ }^{98}$ R. Ryutin, ${ }^{98}$ A. Sobol, ${ }^{98}$ L. Tourtchanovitch, ${ }^{98}$ S. Troshin, ${ }^{98}$ N. Tyurin, ${ }^{98}$ A. Uzunian, ${ }^{98}$ A. Volkov, ${ }^{98}$ P. Adzic, ${ }^{99, p p}$ P. Cirkovic, ${ }^{99}$ J. Milosevic, ${ }^{99}$ V. Rekovic, ${ }^{99}$ J. Alcaraz Maestre, ${ }^{100}$ E. Calvo, ${ }^{100}$ M. Cerrada, ${ }^{100}$ M. Chamizo Llatas, ${ }^{100}$ N. Colino, ${ }^{100}$ B. De La Cruz, ${ }^{100}$ A. Delgado Peris, ${ }^{100}$ D. Domínguez Vázquez, ${ }^{100}$ A. Escalante Del Valle, ${ }^{100}$ C. Fernandez Bedoya, ${ }^{100}$ J. P. Fernández Ramos, ${ }^{100}$ J. Flix, ${ }^{100}$ M. C. Fouz, ${ }^{100}$ P. Garcia-Abia, ${ }^{100}$ O. Gonzalez Lopez, ${ }^{100}$ S. Goy Lopez, ${ }^{100}$ J. M. Hernandez, ${ }^{100}$ M. I. Josa, ${ }^{100}$ E. Navarro De Martino, ${ }^{100}$ A. Pérez-Calero Yzquierdo, ${ }^{100}$ J. Puerta Pelayo, ${ }^{100}$ A. Quintario Olmeda,${ }^{100}$ I. Redondo, ${ }^{100}$ L. Romero, ${ }^{100}$ J. Santaolalla, ${ }^{100}$ M. S. Soares, ${ }^{100}$ C. Albajar, ${ }^{101}$ J. F. de Trocóniz, ${ }^{101}$ M. Missiroli, ${ }^{101}$ D. Moran, ${ }^{101}$ J. Cuevas, ${ }^{102}$ J. Fernandez Menendez, ${ }^{102}$ S. Folgueras, ${ }^{102}$ I. Gonzalez Caballero, ${ }^{102}$ E. Palencia Cortezon, ${ }^{102}$ J. M. Vizan Garcia, ${ }^{102}$ I. J. Cabrillo, ${ }^{103}$ A. Calderon, ${ }^{103}$ J. R. Castiñeiras De Saa, ${ }^{103}$ P. De Castro Manzano, ${ }^{103}$ M. Fernandez, ${ }^{103}$ J. Garcia-Ferrero, ${ }^{103}$ G. Gomez, ${ }^{103}$ A. Lopez Virto, ${ }^{103}$ J. Marco,${ }^{103}$ R. Marco, ${ }^{103}$ C. Martinez Rivero, ${ }^{103}$ F. Matorras, ${ }^{103}$ J. Piedra Gomez, ${ }^{103}$ T. Rodrigo, ${ }^{103}$ A. Y. Rodríguez-Marrero, ${ }^{103}$ A. Ruiz-Jimeno, ${ }^{103}$ L. Scodellaro,${ }^{103}$ N. Trevisani, ${ }^{103}$ I. Vila, ${ }^{103}$ R. Vilar Cortabitarte,${ }^{103}$ D. Abbaneo, ${ }^{104}$ E. Auffray, ${ }^{104}$ G. Auzinger, ${ }^{104}$ M. Bachtis, ${ }^{104}$ P. Baillon, ${ }^{104}$ A. H. Ball, ${ }^{104}$ D. Barney, ${ }^{104}$ A. Benaglia, ${ }^{104}$ J. Bendavid, ${ }^{104}$ L. Benhabib, ${ }^{104}$ J. F. Benitez, ${ }^{104}$ G. M. Berruti, ${ }^{104}$ P. Bloch,${ }^{104}$ A. Bocci, ${ }^{104}$ A. Bonato, ${ }^{104}$ C. Botta, ${ }^{104}$ H. Breuker, ${ }^{104}$ T. Camporesi, ${ }^{104}$ R. Castello, ${ }^{104}$ G. Cerminara, ${ }^{104}$ M. D'Alfonso, ${ }^{104}$ D. d'Enterria, ${ }^{104}$ A. Dabrowski, ${ }^{104}$ V. Daponte, ${ }^{104}$ A. David,${ }^{104}$ M. De Gruttola, ${ }^{104}$ F. De Guio, ${ }^{104}$ A. De Roeck, ${ }^{104}$ S. De Visscher, ${ }^{104}$ E. Di Marco, ${ }^{104, q q}$ M. Dobson, ${ }^{104}$ M. Dordevic, ${ }^{104}$ B. Dorney, ${ }^{104}$ T. du Pree, ${ }^{104}$ D. Duggan, ${ }^{104}$ M. Dünser, ${ }^{104}$ N. Dupont, ${ }^{104}$ A. Elliott-Peisert, ${ }^{104}$ G. Franzoni, ${ }^{104}$ J. Fulcher, ${ }^{104}$ W. Funk, ${ }^{104}$ D. Gigi, ${ }^{104}$ K. Gill, ${ }^{104}$ D. Giordano, ${ }^{104}$ M. Girone, ${ }^{104}$ F. Glege,${ }^{104}$ R. Guida, ${ }^{104}$ S. Gundacker, ${ }^{104}$ M. Guthoff, ${ }^{104}$ J. Hammer, ${ }^{104}$ P. Harris, ${ }^{104}$ J. Hegeman, ${ }^{104}$ V. Innocente, ${ }^{104}$ P. Janot,${ }^{104}$ H. Kirschenmann, ${ }^{104}$ M. J. Kortelainen, ${ }^{104}$ K. Kousouris, ${ }^{104}$ K. Krajczar, ${ }^{104}$ P. Lecoq ${ }^{104}$ C. Lourenço, ${ }^{104}$ M. T. Lucchini, ${ }^{104}$ N. Magini, ${ }^{104}$ L. Malgeri, ${ }^{104}$ M. Mannelli, ${ }^{104}$ A. Martelli, ${ }^{104}$ L. Masetti, ${ }^{104}$ F. Meijers, ${ }^{104}$ S. Mersi,${ }^{104}$ E. Meschi, ${ }^{104}$ F. Moortgat, ${ }^{104}$ S. Morovic, ${ }^{104}$ M. Mulders, ${ }^{104}$ M. V. Nemallapudi, ${ }^{104}$ H. Neugebauer, ${ }^{104}$ S. Orfanelli, ${ }^{104, r r}$ L. Orsini, ${ }^{104}$ L. Pape, ${ }^{104}$ E. Perez,${ }^{104}$ M. Peruzzi,${ }^{104}$ A. Petrilli, ${ }^{104}$ G. Petrucciani, ${ }^{104}$ A. Pfeiffer, ${ }^{104}$ D. Piparo, ${ }^{104}$ A. Racz, ${ }^{104}$ T. Reis, ${ }^{104}$ G. Rolandi ${ }^{104, s s}$ M. Rovere, ${ }^{104}$ M. Ruan, ${ }^{104}$ H. Sakulin, ${ }^{104}$ C. Schäfer, ${ }^{104}$ C. Schwick,${ }^{104}$ M. Seidel,${ }^{104}$ A. Sharma, ${ }^{104}$ P. Silva, ${ }^{104}$ M. Simon, ${ }^{104}$ P. Sphicas, ${ }^{104, t t}$ J. Steggemann, ${ }^{104}$ B. Stieger, ${ }^{104}$ M. Stoye, ${ }^{104}$ Y. Takahashi, ${ }^{104}$ D. Treille,${ }^{104}$ A. Triossi, ${ }^{104}$ A. Tsirou, ${ }^{104}$ G. I. Veres, ${ }^{104, w}$ N. Wardle, ${ }^{104}$ H. K. Wöhri, ${ }^{104}$ A. Zagozdzinska, ${ }^{104, k k}$ W. D. Zeuner, ${ }^{104}$ W. Bertl, ${ }^{105}$ K. Deiters, ${ }^{105}$ W. Erdmann, ${ }^{105}$ R. Horisberger, ${ }^{105}$ Q. Ingram, ${ }^{105}$ H. C. Kaestli, ${ }^{105}$ D. Kotlinski, ${ }^{105}$ U. Langenegger, ${ }^{105}$ D. Renker, ${ }^{105}$ T. Rohe, ${ }^{105}$ F. Bachmair, ${ }^{106}$ L. Bäni ${ }^{106}$ L. Bianchini, ${ }^{106}$ B. Casal, ${ }^{106}$ G. Dissertori, ${ }^{106}$ M. Dittmar, ${ }^{106}$ M. Donegà, ${ }^{106}$ P. Eller, ${ }^{106}$ C. Grab,${ }^{106}$ C. Heidegger, ${ }^{106}$ D. Hits, ${ }^{106}$ J. Hoss, ${ }^{106}$ G. Kasieczka, ${ }^{106}$ W. Lustermann, ${ }^{106}$ B. Mangano, ${ }^{106}$ M. Marionneau, ${ }^{106}$ P. Martinez Ruiz del Arbol, ${ }^{106}$ M. Masciovecchio, ${ }^{106}$ D. Meister, ${ }^{106}$ F. Micheli, ${ }^{106}$ P. Musella, ${ }^{106}$ F. Nessi-Tedaldi, ${ }^{106}$ F. Pandolfi, ${ }^{106}$ J. Pata,${ }^{106}$ F. Pauss, ${ }^{106}$ L. Perrozzi, ${ }^{106}$ M. Quittnat, ${ }^{106}$ M. Rossini, ${ }^{106}$ A. Starodumov, ${ }^{106, \text { uu }}$ M. Takahashi, ${ }^{106}$ V. R. Tavolaro, ${ }^{106}$ K. Theofilatos, ${ }^{106}$ R. Wallny, ${ }^{106}$ T. K. Aarrestad, ${ }^{107}$ C. Amsler ${ }^{107, \mathrm{vv}}$ L. Caminada, ${ }^{107}$ M. F. Canelli, ${ }^{107}$ V. Chiochia,${ }^{107}$ A. De Cosa,${ }^{107}$ C. Galloni, ${ }^{107}$ A. Hinzmann, ${ }^{107}$

T. Hreus,${ }^{107}$ B. Kilminster, ${ }^{107}$ C. Lange, ${ }^{107}$ J. Ngadiuba, ${ }^{107}$ D. Pinna,${ }^{107}$ P. Robmann, ${ }^{107}$ F. J. Ronga, ${ }^{107}$ D. Salerno, ${ }^{107}$ Y. Yang, ${ }^{107}$ M. Cardaci, ${ }^{108}$ K. H. Chen, ${ }^{108}$ T. H. Doan, ${ }^{108}$ Sh. Jain, ${ }^{108}$ R. Khurana, ${ }^{108}$ M. Konyushikhin, ${ }^{108}$ C. M. Kuo, ${ }^{108}$ W. Lin, ${ }^{108}$ Y. J. Lu, ${ }^{108}$ S. S. Yu, ${ }^{108}$ Arun Kumar, ${ }^{109}$ R. Bartek, ${ }^{109}$ P. Chang, ${ }^{109}$ Y. H. Chang, ${ }^{109}$ Y. W. Chang,,${ }^{109}$ Y. Chao, ${ }^{109}$ K. F. Chen, ${ }^{109}$ P. H. Chen, ${ }^{109}$ C. Dietz, ${ }^{109}$ F. Fiori, ${ }^{109}$ U. Grundler, ${ }^{109}$ W.-S. Hou, ${ }^{109}$ Y. Hsiung, ${ }^{109}$ Y. F. Liu, ${ }^{109}$ R.-S. Lu, ${ }^{109}$ M. Miñano Moya ${ }^{109}$ E. Petrakou, ${ }^{109}$ J. f. Tsai, ${ }^{109}$ Y. M. Tzeng, ${ }^{109}$ B. Asavapibhop, ${ }^{110}$ K. Kovitanggoon,,${ }^{110}$ G. Singh, ${ }^{110}$ N. Srimanobhas,${ }^{110}$ N. Suwonjandee,${ }^{110}$ A. Adiguzel, ${ }^{111}$ S. Cerci, ${ }^{111, w w}$ Z. S. Demiroglu ${ }^{111}$ C. Dozen, ${ }^{111}$ I. Dumanoglu, ${ }^{111}$

S. Girgis, ${ }^{111}$ G. Gokbulut, ${ }^{111}$ Y. Guler,${ }^{111}$ E. Gurpinar, ${ }^{111}$ I. Hos, ${ }^{111}$ E. E. Kangal, ${ }^{111, x x}$ A. Kayis Topaksu, ${ }^{111}$ G. Onengut, ${ }^{11, y y}$ K. Ozdemir, ${ }^{11, z z}$ S. Ozturk, ${ }^{111, \text { aaa }}$ A. Polatoz, ${ }^{111}$ B. Tali, ${ }^{111, w w}$ M. Vergili, ${ }^{111}$ C. Zorbilmez,${ }^{111}$ I. V. Akin, ${ }^{112}$ B. Bilin, ${ }^{112}$ S. Bilmis, ${ }^{112}$ B. Isildak, ${ }^{112, b b b}$ G. Karapinar, ${ }^{112, \text { ccc }}$ M. Yalvac, ${ }^{112}$ M. Zeyrek, ${ }^{112}$ E. Gülmez, ${ }^{113}$ M. Kaya, ${ }^{113, \text { ddd }}$ O. Kaya, ${ }^{113, \text { eee }}$ E. A. Yetkin, ${ }^{11, \text { fff }}$ T. Yetkin, ${ }^{13, \text { ggg }}$ A. Cakir ${ }^{114}$ K. Cankocak, ${ }^{114}$ S. Sen, ${ }^{114, \text { hhh }}$ F. I. Vardarl,,${ }^{114}$ B. Grynyov, ${ }^{115}$ L. Levchuk, ${ }^{116}$ P. Sorokin, ${ }^{116}$ R. Aggleton, ${ }^{117}$ F. Ball, ${ }^{117}$ L. Beck, ${ }^{117}$ J. J. Brooke, ${ }^{117}$ E. Clement, ${ }^{117}$ D. Cussans, ${ }^{117}$ H. Flacher, ${ }^{117}$ J. Goldstein, ${ }^{117}$ M. Grimes,${ }^{117}$ G. P. Heath, ${ }^{117}$ H. F. Heath ${ }^{117}$ J. Jacob ${ }^{117}$ L. Kreczko, ${ }^{117}$ C. Lucas, ${ }^{117}$ Z. Meng, ${ }^{117}$ D. M. Newbold, ${ }^{117, i i i}$ S. Paramesvaran, ${ }^{117}$ A. Poll, ${ }^{117}$ T. Sakuma, ${ }^{117}$ S. Seif El Nasr-storey,${ }^{117}$ S. Senkin, ${ }^{117}$ D. Smith, ${ }^{117}$ V. J. Smith, ${ }^{117}$ K. W. Bell, ${ }^{118}$ A. Belyaev, ${ }^{18, j j j}$ C. Brew, ${ }^{118}$ R. M. Brown, ${ }^{118}$ L. Calligaris, ${ }^{118}$ D. Cieri, ${ }^{18}$ D. J. A. Cockerill, ${ }^{118}$ J. A. Coughlan, ${ }^{118}$ K. Harder,${ }^{118}$ S. Harper, ${ }^{118}$ E. Olaiya, ${ }^{118}$ D. Petyt,${ }^{118}$

C. H. Shepherd-Themistocleous, ${ }^{118}$ A. Thea, ${ }^{118}$ I. R. Tomalin, ${ }^{118}$ T. Williams, ${ }^{118}$ S. D. Worm, ${ }^{118}$ M. Baber, ${ }^{119}$ R. Bainbridge, ${ }^{119}$ O. Buchmuller, ${ }^{119}$ A. Bundock, ${ }^{119}$ D. Burton, ${ }^{119}$ S. Casasso, ${ }^{119}$ M. Citron, ${ }^{119}$ D. Colling, ${ }^{119}$ L. Corpe,${ }^{119}$ 
N. Cripps ${ }^{119}$ P. Dauncey, ${ }^{119}$ G. Davies, ${ }^{119}$ A. De Wit, ${ }^{119}$ M. Della Negra, ${ }^{119}$ P. Dunne, ${ }^{119}$ A. Elwood, ${ }^{119}$ W. Ferguson, ${ }^{119}$ D. Futyan, ${ }^{119}$ G. Hall, ${ }^{119}$ G. Iles, ${ }^{119}$ M. Kenzie, ${ }^{119}$ R. Lane, ${ }^{119}$ R. Lucas, ${ }^{119, \text { iii }}$ L. Lyons, ${ }^{119}$ A.-M. Magnan, ${ }^{119}$ S. Malik, ${ }^{119}$ J. Nash, ${ }^{119}$ A. Nikitenko, ${ }^{119, \text { uu }}$ J. Pela,${ }^{119}$ M. Pesaresi, ${ }^{119}$ K. Petridis, ${ }^{119}$ D. M. Raymond, ${ }^{119}$ A. Richards,${ }^{119}$ A. Rose,${ }^{119}$ C. Seez ${ }^{119}$ A. Tapper, ${ }^{119}$ K. Uchida, ${ }^{119}$ M. Vazquez Acosta, ${ }^{119, k k k}$ T. Virdee,${ }^{119}$ S. C. Zenz, ${ }^{119}$ J. E. Cole,${ }^{120}$ P. R. Hobson, ${ }^{120}$ A. Khan, ${ }^{120}$ P. Kyberd, ${ }^{120}$ D. Leggat,${ }^{120}$ D. Leslie, ${ }^{120}$ I. D. Reid, ${ }^{120}$ P. Symonds, ${ }^{120}$ L. Teodorescu, ${ }^{120}$ M. Turner, ${ }^{120}$

A. Borzou, ${ }^{121}$ K. Call, ${ }^{121}$ J. Dittmann, ${ }^{121}$ K. Hatakeyama, ${ }^{121}$ H. Liu, ${ }^{121}$ N. Pastika, ${ }^{121}$ O. Charaf, ${ }^{122}$ S. I. Cooper, ${ }^{122}$ C. Henderson, ${ }^{122}$ P. Rumerio, ${ }^{122}$ D. Arcaro, ${ }^{123}$ A. Avetisyan, ${ }^{123}$ T. Bose,${ }^{123}$ C. Fantasia, ${ }^{123}$ D. Gastler, ${ }^{123}$ P. Lawson, ${ }^{123}$ D. Rankin, ${ }^{123}$ C. Richardson, ${ }^{123}$ J. Rohlf, ${ }^{123}$ J. St. John, ${ }^{123}$ L. Sulak, ${ }^{123}$ D. Zou, ${ }^{123}$ J. Alimena, ${ }^{124}$ E. Berry, ${ }^{124}$ S. Bhattacharya ${ }^{124}$ D. Cutts, ${ }^{124}$ N. Dhingra, ${ }^{124}$ A. Ferapontov,${ }^{124}$ A. Garabedian, ${ }^{124}$ J. Hakala, ${ }^{124}$ U. Heintz,${ }^{124}$ E. Laird, ${ }^{124}$ G. Landsberg, ${ }^{124}$ Z. Mao, ${ }^{124}$ M. Narain, ${ }^{124}$ S. Piperov, ${ }^{124}$ S. Sagir, ${ }^{124}$ R. Syarif, ${ }^{124}$ R. Breedon, ${ }^{125}$ G. Breto, ${ }^{125}$ M. Calderon De La Barca Sanchez, ${ }^{125}$ S. Chauhan, ${ }^{125}$ M. Chertok, ${ }^{125}$ J. Conway, ${ }^{125}$ R. Conway, ${ }^{125}$ P. T. Cox, ${ }^{125}$ R. Erbacher, ${ }^{125}$ M. Gardner, ${ }^{125}$ W. Ko, ${ }^{125}$ R. Lander, ${ }^{125}$ M. Mulhearn, ${ }^{125}$ D. Pellett, ${ }^{125}$ J. Pilot, ${ }^{125}$ F. Ricci-Tam, ${ }^{125}$ S. Shalhout, ${ }^{125}$ J. Smith ${ }^{125}$ M. Squires, ${ }^{125}$ D. Stolp,${ }^{125}$ M. Tripathi, ${ }^{125}$ S. Wilbur, ${ }^{125}$ R. Yohay,${ }^{125}$ C. Bravo, ${ }^{126}$ R. Cousins, ${ }^{126}$ P. Everaerts, ${ }^{126}$ C. Farrell, ${ }^{126}$ A. Florent, ${ }^{126}$ J. Hauser, ${ }^{126}$ M. Ignatenko, ${ }^{126}$ D. Saltzberg, ${ }^{126}$ E. Takasugi, ${ }^{126}$ V. Valuev, ${ }^{126}$ M. Weber, ${ }^{126}$ K. Burt, ${ }^{127}$ R. Clare, ${ }^{127}$ J. Ellison, ${ }^{127}$ J. W. Gary, ${ }^{127}$ G. Hanson, ${ }^{127}$ J. Heilman, ${ }^{127}$ M. Ivova PANEVA, ${ }^{127}$ P. Jandir, ${ }^{127}$ E. Kennedy, ${ }^{127}$ F. Lacroix, ${ }^{127}$ O. R. Long, ${ }^{127}$ A. Luthra, ${ }^{127}$ M. Malberti, ${ }^{127}$ M. Olmedo Negrete,${ }^{127}$

A. Shrinivas, ${ }^{127}$ H. Wei ${ }^{127}$ S. Wimpenny, ${ }^{127}$ B. R. Yates, ${ }^{127}$ J. G. Branson, ${ }^{128}$ G. B. Cerati, ${ }^{128}$ S. Cittolin, ${ }^{128}$ R. T. D'Agnolo, ${ }^{128}$ M. Derdzinski, ${ }^{128}$ A. Holzner, ${ }^{128}$ R. Kelley, ${ }^{128}$ D. Klein, ${ }^{128}$ J. Letts, ${ }^{128}$ I. Macneill, ${ }^{128}$ D. Olivito, ${ }^{128}$ S. Padhi, ${ }^{128}$ M. Pieri, ${ }^{128}$ M. Sani, ${ }^{128}$ V. Sharma, ${ }^{128}$ S. Simon, ${ }^{128}$ M. Tadel, ${ }^{128}$ A. Vartak, ${ }^{128}$ S. Wasserbaech, ${ }^{128,111}$ C. Welke, ${ }^{128}$ F. Würthwein, ${ }^{128}$ A. Yagil, ${ }^{128}$ G. Zevi Della Porta, ${ }^{128}$ J. Bradmiller-Feld, ${ }^{129}$ C. Campagnari, ${ }^{129}$ A. Dishaw, ${ }^{129}$ V. Dutta, ${ }^{129}$ K. Flowers, ${ }^{129}$ M. Franco Sevilla, ${ }^{129}$ P. Geffert, ${ }^{129}$ C. George,${ }^{129}$ F. Golf, ${ }^{129}$ L. Gouskos, ${ }^{129}$ J. Gran, ${ }^{129}$ J. Incandela, ${ }^{129}$ N. Mccoll, ${ }^{129}$ S. D. Mullin, ${ }^{129}$ J. Richman, ${ }^{129}$ D. Stuart, ${ }^{129}$ I. Suarez,${ }^{129}$ C. West,${ }^{129}$ J. Yoo, ${ }^{129}$ D. Anderson, ${ }^{130}$ A. Apresyan, ${ }^{130}$ A. Bornheim, ${ }^{130}$ J. Bunn, ${ }^{130}$ Y. Chen, ${ }^{130}$ J. Duarte, ${ }^{130}$ A. Mott, ${ }^{130}$ H. B. Newman, ${ }^{130}$ C. Pena, ${ }^{130}$ M. Pierini, ${ }^{130}$ M. Spiropulu, ${ }^{130}$ J. R. Vlimant, ${ }^{130}$ S. Xie, ${ }^{130}$ R. Y. Zhu, ${ }^{130}$ M. B. Andrews, ${ }^{131}$ V. Azzolini, ${ }^{131}$ A. Calamba, ${ }^{131}$ B. Carlson, ${ }^{131}$ T. Ferguson, ${ }^{131}$ M. Paulini, ${ }^{131}$ J. Russ, ${ }^{131}$ M. Sun, ${ }^{131}$ H. Vogel, ${ }^{131}$ I. Vorobiev, ${ }^{131}$ J. P. Cumalat, ${ }^{132}$ W. T. Ford, ${ }^{132}$ A. Gaz, ${ }^{132}$ F. Jensen, ${ }^{132}$ A. Johnson, ${ }^{132}$ M. Krohn, ${ }^{132}$ T. Mulholland, ${ }^{132}$ U. Nauenberg, ${ }^{132}$ K. Stenson, ${ }^{132}$ S. R. Wagner, ${ }^{132}$ J. Alexander, ${ }^{133}$ A. Chatterjee, ${ }^{133}$ J. Chaves, ${ }^{133}$ J. Chu, ${ }^{133}$ S. Dittmer, ${ }^{133}$ N. Eggert, ${ }^{133}$ N. Mirman, ${ }^{133}$ G. Nicolas Kaufman, ${ }^{133}$ J. R. Patterson, ${ }^{133}$ A. Rinkevicius, ${ }^{133}$ A. Ryd, ${ }^{133}$ L. Skinnari, ${ }^{133}$ L. Soffi, ${ }^{133}$ W. Sun, ${ }^{133}$ S. M. Tan, ${ }^{133}$ W. D. Teo, ${ }^{133}$ J. Thom, ${ }^{133}$ J. Thompson, ${ }^{133}$ J. Tucker, ${ }^{133}$ Y. Weng, ${ }^{133}$ P. Wittich, ${ }^{133}$ S. Abdullin, ${ }^{134}$ M. Albrow, ${ }^{134}$ G. Apollinari, ${ }^{134}$ S. Banerjee, ${ }^{134}$ L. A. T. Bauerdick, ${ }^{134}$ A. Beretvas, ${ }^{134}$ J. Berryhill, ${ }^{134}$ P. C. Bhat, ${ }^{134}$ G. Bolla, ${ }^{134}$ K. Burkett, ${ }^{134}$ J. N. Butler, ${ }^{134}$ H. W. K. Cheung, ${ }^{134}$ F. Chlebana, ${ }^{134}$ S. Cihangir, ${ }^{134}$ V. D. Elvira, ${ }^{134}$ I. Fisk, ${ }^{134}$ J. Freeman, ${ }^{134}$ E. Gottschalk, ${ }^{134}$ L. Gray, ${ }^{134}$ D. Green, ${ }^{134}$ S. Grünendahl, ${ }^{134}$ O. Gutsche, ${ }^{134}$ J. Hanlon, ${ }^{134}$ D. Hare, ${ }^{134}$ R. M. Harris, ${ }^{134}$ S. Hasegawa, ${ }^{134}$ J. Hirschauer, ${ }^{134}$ Z. Hu, ${ }^{134}$ B. Jayatilaka, ${ }^{134}$ S. Jindariani, ${ }^{134}$ M. Johnson, ${ }^{134}$ U. Joshi, ${ }^{134}$ A. W. Jung, ${ }^{134}$ B. Klima, ${ }^{134}$ B. Kreis, ${ }^{134}$ S. Lammel, ${ }^{134}$ J. Linacre, ${ }^{134}$ D. Lincoln,${ }^{134}$ R. Lipton, ${ }^{134}$ T. Liu, ${ }^{134}$ R. Lopes De Sá, ${ }^{134}$ J. Lykken, ${ }^{134}$ K. Maeshima, ${ }^{134}$ J. M. Marraffino, ${ }^{134}$ V. I. Martinez Outschoorn, ${ }^{134}$ S. Maruyama, ${ }^{134}$ D. Mason, ${ }^{134}$ P. McBride, ${ }^{134}$ P. Merkel, ${ }^{134}$ K. Mishra, ${ }^{134}$ S. Mrenna, ${ }^{134}$ S. Nahn, ${ }^{134}$ C. Newman-Holmes, ${ }^{134}$ V. O’Dell ${ }^{134}$ K. Pedro, ${ }^{134}$ O. Prokofyev, ${ }^{134}$ G. Rakness, ${ }^{134}$ E. Sexton-Kennedy, ${ }^{134}$ A. Soha, ${ }^{134}$ W. J. Spalding, ${ }^{134}$ L. Spiegel, ${ }^{134}$ N. Strobbe ${ }^{134}$ L. Taylor, ${ }^{134}$

S. Tkaczyk,${ }^{134}$ N. V. Tran, ${ }^{134}$ L. Uplegger,${ }^{134}$ E. W. Vaandering,${ }^{134}$ C. Vernieri, ${ }^{134}$ M. Verzocchi, ${ }^{134}$ R. Vidal, ${ }^{134}$ H. A. Weber, ${ }^{134}$ A. Whitbeck, ${ }^{134}$ D. Acosta, ${ }^{135}$ P. Avery, ${ }^{135}$ P. Bortignon, ${ }^{135}$ D. Bourilkov, ${ }^{135}$ A. Carnes, ${ }^{135}$ M. Carver, ${ }^{135}$ D. Curry, ${ }^{135}$ S. Das, ${ }^{135}$ R. D. Field, ${ }^{135}$ I. K. Furic, ${ }^{135}$ S. V. Gleyzer, ${ }^{135}$ J. Hugon, ${ }^{135}$ J. Konigsberg, ${ }^{135}$ A. Korytov, ${ }^{135}$ J. F. Low, ${ }^{135}$ P. Ma, ${ }^{135}$ K. Matchev, ${ }^{135}$ H. Mei ${ }^{135}$ P. Milenovic, ${ }^{135, m m m}$ G. Mitselmakher, ${ }^{135}$ D. Rank, ${ }^{135}$ R. Rossin, ${ }^{135}$ L. Shchutska, ${ }^{135}$ M. Snowball, ${ }^{135}$ D. Sperka, ${ }^{135}$ N. Terentyev, ${ }^{135}$ L. Thomas, ${ }^{135}$ J. Wang, ${ }^{135}$ S. Wang, ${ }^{135}$ J. Yelton, ${ }^{135}$ S. Hewamanage, ${ }^{136}$ S. Linn, ${ }^{136}$ P. Markowitz, ${ }^{136}$ G. Martinez, ${ }^{136}$ J. L. Rodriguez,${ }^{136}$ A. Ackert, ${ }^{137}$ J. R. Adams, ${ }^{137}$ T. Adams, ${ }^{137}$ A. Askew, ${ }^{137}$ S. Bein, ${ }^{137}$ J. Bochenek, ${ }^{137}$ B. Diamond, ${ }^{137}$ J. Haas ${ }^{137}$ S. Hagopian, ${ }^{137}$ V. Hagopian, ${ }^{137}$ K. F. Johnson, ${ }^{137}$ A. Khatiwada, ${ }^{137}$ H. Prosper, ${ }^{137}$ M. Weinberg, ${ }^{137}$ M. M. Baarmand, ${ }^{138}$ V. Bhopatkar, ${ }^{138}$ S. Colafranceschi, ${ }^{138, n n n}$ M. Hohlmann, ${ }^{138}$ H. Kalakhety, ${ }^{138}$ D. Noonan, ${ }^{138}$ T. Roy, ${ }^{138}$ F. Yumiceva, ${ }^{138}$ M. R. Adams, ${ }^{139}$ L. Apanasevich, ${ }^{139}$ D. Berry, ${ }^{139}$ R. R. Betts, ${ }^{139}$ I. Bucinskaite, ${ }^{139}$ R. Cavanaugh, ${ }^{139}$ O. Evdokimov, ${ }^{139}$ L. Gauthier, ${ }^{139}$ C. E. Gerber, ${ }^{139}$ D. J. Hofman, ${ }^{139}$ P. Kurt, ${ }^{139}$ C. O'Brien, ${ }^{139}$ I. D. Sandoval Gonzalez, ${ }^{139}$ C. Silkworth, ${ }^{139}$ P. Turner, ${ }^{139}$ N. Varelas, ${ }^{139}$ Z. Wu, ${ }^{139}$ M. Zakaria, ${ }^{139}$ B. Bilki, ${ }^{140,000}$ W. Clarida, ${ }^{140}$ K. Dilsiz, ${ }^{140}$ S. Durgut,${ }^{140}$ R. P. Gandrajula, ${ }^{140}$ 
M. Haytmyradov ${ }^{140}$ V. Khristenko, ${ }^{140}$ J.-P. Merlo, ${ }^{140}$ H. Mermerkaya, ${ }^{140, p p p}$ A. Mestvirishvili, ${ }^{140}$ A. Moeller, ${ }^{140}$ J. Nachtman, ${ }^{140}$ H. Ogul, ${ }^{140}$ Y. Onel,${ }^{140}$ F. Ozok, ${ }^{140, f f f}$ A. Penzo, ${ }^{140}$ C. Snyder, ${ }^{140}$ E. Tiras, ${ }^{140}$ J. Wetzel, ${ }^{140}$ K. Yi, ${ }^{140}$ I. Anderson, ${ }^{141}$ B. A. Barnett, ${ }^{141}$ B. Blumenfeld, ${ }^{141}$ N. Eminizer, ${ }^{141}$ D. Fehling, ${ }^{141}$ L. Feng, ${ }^{141}$ A. V. Gritsan, ${ }^{141}$ P. Maksimovic, ${ }^{141}$ C. Martin, ${ }^{141}$ M. Osherson, ${ }^{141}$ J. Roskes, ${ }^{141}$ A. Sady,${ }^{141}$ U. Sarica, ${ }^{141}$ M. Swartz, ${ }^{141}$ M. Xiao, ${ }^{141}$ Y. Xin, ${ }^{141}$ C. You, ${ }^{141}$ P. Baringer, ${ }^{142}$ A. Bean,${ }^{142}$ G. Benelli, ${ }^{142}$ C. Bruner, ${ }^{142}$ R. P. Kenny III, ${ }^{142}$ D. Majumder, ${ }^{142}$ M. Malek,${ }^{142}$ M. Murray ${ }^{142}$ S. Sanders, ${ }^{142}$ R. Stringer, ${ }^{142}$ Q. Wang, ${ }^{142}$ A. Ivanov, ${ }^{143}$ K. Kaadze, ${ }^{143}$ S. Khalil,${ }^{143}$ M. Makouski, ${ }^{143}$ Y. Maravin, ${ }^{143}$ A. Mohammadi, ${ }^{143}$ L. K. Saini,${ }^{143}$ N. Skhirtladze, ${ }^{143}$ S. Toda,${ }^{143}$ D. Lange ${ }^{144}$ F. Rebassoo, ${ }^{144}$ D. Wright, ${ }^{144}$ C. Anelli, ${ }^{145}$ A. Baden, ${ }^{145}$ O. Baron, ${ }^{145}$ A. Belloni, ${ }^{145}$ B. Calvert, ${ }^{145}$ S. C. Eno, ${ }^{145}$ C. Ferraioli, ${ }^{145}$ J. A. Gomez ${ }^{145}$ N. J. Hadley, ${ }^{145}$ S. Jabeen, ${ }^{145}$ R. G. Kellogg, ${ }^{145}$ T. Kolberg, ${ }^{145}$ J. Kunkle, ${ }^{145}$ Y. Lu, ${ }^{145}$ A. C. Mignerey, ${ }^{145}$ Y. H. Shin, ${ }^{145}$ A. Skuja, ${ }^{145}$ M. B. Tonjes, ${ }^{145}$ S. C. Tonwar, ${ }^{145}$ A. Apyan, ${ }^{146}$ R. Barbieri, ${ }^{146}$ A. Baty,${ }^{146}$ K. Bierwagen, ${ }^{146}$ S. Brandt, ${ }^{146}$ W. Busza, ${ }^{146}$ I. A. Cali, ${ }^{146}$ Z. Demiragli, ${ }^{146}$ L. Di Matteo, ${ }^{146}$ G. Gomez Ceballos, ${ }^{146}$ M. Goncharov,${ }^{146}$ D. Gulhan, ${ }^{146}$ Y. Iiyama, ${ }^{146}$ G. M. Innocenti, ${ }^{146}$ M. Klute, ${ }^{146}$ D. Kovalskyi, ${ }^{146}$ Y. S. Lai, ${ }^{146}$ Y.-J. Lee, ${ }^{146}$ A. Levin, ${ }^{146}$ P. D. Luckey, ${ }^{146}$ A. C. Marini, ${ }^{146}$ C. Mcginn, ${ }^{146}$ C. Mironov, ${ }^{146}$ S. Narayanan, ${ }^{146}$ X. Niu, ${ }^{146}$ C. Paus, ${ }^{146}$ D. Ralph, ${ }^{146}$ C. Roland, ${ }^{146}$ G. Roland, ${ }^{146}$ J. Salfeld-Nebgen, ${ }^{146}$ G. S. F. Stephans, ${ }^{146}$ K. Sumorok, ${ }^{146}$ M. Varma, ${ }^{146}$ D. Velicanu, ${ }^{146}$ J. Veverka, ${ }^{146}$ J. Wang, ${ }^{146}$ T. W. Wang, ${ }^{146}$ B. Wyslouch, ${ }^{146}$ M. Yang, ${ }^{146}$ V. Zhukova, ${ }^{146}$ B. Dahmes, ${ }^{147}$ A. Evans, ${ }^{147}$ A. Finkel, ${ }^{147}$ A. Gude, ${ }^{147}$ P. Hansen, ${ }^{147}$ S. Kalafut, ${ }^{147}$ S. C. Kao, ${ }^{147}$ K. Klapoetke, ${ }^{147}$ Y. Kubota,${ }^{147}$ Z. Lesko, ${ }^{147}$ J. Mans, ${ }^{147}$ S. Nourbakhsh,${ }^{147}$ N. Ruckstuhl, ${ }^{147}$ R. Rusack ${ }^{147}$ N. Tambe, ${ }^{147}$ J. Turkewitz ${ }^{147}$ J. G. Acosta, ${ }^{148}$ S. Oliveros, ${ }^{148}$ E. Avdeeva, ${ }^{149}$ K. Bloom, ${ }^{149}$ S. Bose,${ }^{149}$ D. R. Claes, ${ }^{149}$ A. Dominguez,${ }^{149}$ C. Fangmeier ${ }^{149}$ R. Gonzalez Suarez,${ }^{149}$ R. Kamalieddin, ${ }^{149}$ J. Keller, ${ }^{149}$ D. Knowlton, ${ }^{149}$ I. Kravchenko, ${ }^{149}$ F. Meier, ${ }^{149}$ J. Monroy, ${ }^{149}$ F. Ratnikov, ${ }^{149}$ J. E. Siado, ${ }^{149}$ G. R. Snow, ${ }^{149}$ M. Alyari, ${ }^{150}$ J. Dolen, ${ }^{150}$ J. George,${ }^{150}$ A. Godshalk, ${ }^{150}$ C. Harrington, ${ }^{150}$ I. Iashvili, ${ }^{150}$ J. Kaisen, ${ }^{150}$ A. Kharchilava, ${ }^{150}$ A. Kumar, ${ }^{150}$ S. Rappoccio, ${ }^{150}$ B. Roozbahani, ${ }^{150}$ G. Alverson, ${ }^{151}$ E. Barberis, ${ }^{151}$ D. Baumgartel,,${ }^{151}$ M. Chasco, ${ }^{151}$ A. Hortiangtham, ${ }^{151}$ A. Massironi,${ }^{151}$ D. M. Morse, ${ }^{151}$ D. Nash,${ }^{151}$ T. Orimoto, ${ }^{151}$ R. Teixeira De Lima, ${ }^{151}$ D. Trocino, ${ }^{151}$ R.-J. Wang, ${ }^{151}$ D. Wood, ${ }^{151}$ J. Zhang, ${ }^{151}$ K. A. Hahn, ${ }^{152}$ A. Kubik,${ }^{152}$ N. Mucia,${ }^{152}$ N. Odell, ${ }^{152}$ B. Pollack,${ }^{152}$ A. Pozdnyakov, ${ }^{152}$ M. Schmitt, ${ }^{152}$ S. Stoynev, ${ }^{152}$ K. Sung, ${ }^{152}$ M. Trovato, ${ }^{152}$ M. Velasco, ${ }^{152}$ A. Brinkerhoff, ${ }^{153}$ N. Dev, ${ }^{153}$ M. Hildreth, ${ }^{153}$ C. Jessop, ${ }^{153}$ D. J. Karmgard, ${ }^{153}$ N. Kellams, ${ }^{153}$ K. Lannon, ${ }^{153}$ N. Marinelli, ${ }^{153}$ F. Meng, ${ }^{153}$ C. Mueller, ${ }^{153}$ Y. Musienko, ${ }^{153,11}$ M. Planer, ${ }^{153}$ A. Reinsvold, ${ }^{153}$ R. Ruchti, ${ }^{153}$ G. Smith,${ }^{153}$ S. Taroni, ${ }^{153}$ N. Valls, ${ }^{153}$ M. Wayne, ${ }^{153}$ M. Wolf, ${ }^{153}$ A. Woodard ${ }^{153}$ L. Antonelli, ${ }^{154}$ J. Brinson, ${ }^{154}$ B. Bylsma ${ }^{154}$ L. S. Durkin, ${ }^{154}$ S. Flowers, ${ }^{154}$ A. Hart, ${ }^{154}$

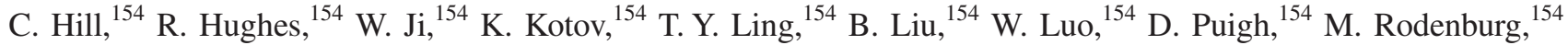
B. L. Winer, ${ }^{154}$ H. W. Wulsin, ${ }^{154}$ O. Driga, ${ }^{155}$ P. Elmer, ${ }^{155}$ J. Hardenbrook, ${ }^{155}$ P. Hebda ${ }^{155}$ S. A. Koay, ${ }^{155}$ P. Lujan, ${ }^{155}$ D. Marlow, ${ }^{155}$ T. Medvedeva, ${ }^{155}$ M. Mooney, ${ }^{155}$ J. Olsen, ${ }^{155}$ C. Palmer, ${ }^{155}$ P. Piroué, ${ }^{155}$ H. Saka, ${ }^{155}$ D. Stickland, ${ }^{155}$ C. Tully, ${ }^{155}$ A. Zuranski, ${ }^{155}$ S. Malik, ${ }^{156}$ V. E. Barnes, ${ }^{157}$ D. Benedetti, ${ }^{157}$ D. Bortoletto, ${ }^{157}$ L. Gutay, ${ }^{157}$ M. K. Jha, ${ }^{157}$ M. Jones, ${ }^{157}$ K. Jung, ${ }^{157}$ D. H. Miller ${ }^{157}$ N. Neumeister, ${ }^{157}$ B. C. Radburn-Smith, ${ }^{157}$ X. Shi, ${ }^{157}$ I. Shipsey, ${ }^{157}$ D. Silvers, ${ }^{157}$ J. Sun, ${ }^{157}$ A. Svyatkovskiy, ${ }^{157}$ F. Wang, ${ }^{157}$ W. Xie,${ }^{157}$ L. Xu, ${ }^{157}$ N. Parashar, ${ }^{158}$ J. Stupak, ${ }^{158}$ A. Adair, ${ }^{159}$ B. Akgun, ${ }^{159}$ Z. Chen, ${ }^{159}$ K. M. Ecklund ${ }^{159}$ F. J. M. Geurts, ${ }^{159}$ M. Guilbaud, ${ }^{159}$ W. Li,${ }^{159}$ B. Michlin, ${ }^{159}$ M. Northup, ${ }^{159}$ B. P. Padley, ${ }^{159}$ R. Redjimi, ${ }^{159}$ J. Roberts, ${ }^{159}$ J. Rorie, ${ }^{159}$ Z. Tu, ${ }^{159}$ J. Zabel, ${ }^{159}$ B. Betchart ${ }^{160}$ A. Bodek, ${ }^{160}$ P. de Barbaro, ${ }^{160}$ R. Demina, ${ }^{160}$ Y. Eshaq, ${ }^{160}$ T. Ferbel, ${ }^{160}$ M. Galanti, ${ }^{160}$ A. Garcia-Bellido, ${ }^{160}$ J. Han, ${ }^{160}$ A. Harel, ${ }^{160}$ O. Hindrichs, ${ }^{160}$ A. Khukhunaishvili, ${ }^{160}$ G. Petrillo, ${ }^{160}$ P. Tan, ${ }^{160}$ M. Verzetti, ${ }^{160}$ S. Arora, ${ }^{161}$ A. Barker, ${ }^{161}$ J. P. Chou, ${ }^{161}$

C. Contreras-Campana, ${ }^{161}$ E. Contreras-Campana, ${ }^{161}$ D. Ferencek, ${ }^{161}$ Y. Gershtein, ${ }^{161}$ R. Gray, ${ }^{161}$ E. Halkiadakis, ${ }^{161}$ D. Hidas, ${ }^{161}$ E. Hughes, ${ }^{161}$ S. Kaplan, ${ }^{161}$ R. Kunnawalkam Elayavalli, ${ }^{161}$ A. Lath, ${ }^{161}$ K. Nash, ${ }^{161}$ S. Panwalkar, ${ }^{161}$ M. Park, ${ }^{161}$ S. Salur, ${ }^{161}$ S. Schnetzer, ${ }^{161}$ D. Sheffield ${ }^{161}$ S. Somalwar, ${ }^{161}$ R. Stone, ${ }^{161}$ S. Thomas, ${ }^{161}$ P. Thomassen, ${ }^{161}$ M. Walker, ${ }^{161}$ M. Foerster, ${ }^{162}$ G. Riley, ${ }^{162}$ K. Rose, ${ }^{162}$ S. Spanier, ${ }^{162}$ A. York, ${ }^{162}$ O. Bouhali, ${ }^{163, q q q}$

A. Castaneda Hernandez, ${ }^{163, q q q}$ A. Celik, ${ }^{163}$ M. Dalchenko, ${ }^{163}$ M. De Mattia, ${ }^{163}$ A. Delgado, ${ }^{163}$ S. Dildick, ${ }^{163}$ R. Eusebi, ${ }^{163}$ J. Gilmore, ${ }^{163}$ T. Huang, ${ }^{163}$ T. Kamon, ${ }^{163, \text { rrr }}$ V. Krutelyov, ${ }^{163}$ R. Mueller, ${ }^{163}$ I. Osipenkov,${ }^{163}$ Y. Pakhotin, ${ }^{163}$ R. Patel, ${ }^{163}$ A. Perloff, ${ }^{163}$ A. Rose, ${ }^{163}$ A. Safonov, ${ }^{163}$ A. Tatarinov, ${ }^{163}$ K. A. Ulmer, ${ }^{163, c}$ N. Akchurin, ${ }^{164}$ C. Cowden, ${ }^{164}$ J. Damgov, ${ }^{164}$ C. Dragoiu, ${ }^{164}$ P. R. Dudero, ${ }^{164}$ J. Faulkner, ${ }^{164}$ S. Kunori, ${ }^{164}$ K. Lamichhane, ${ }^{164}$ S. W. Lee, ${ }^{164}$ T. Libeiro, ${ }^{164}$ S. Undleeb, ${ }^{164}$ I. Volobouev, ${ }^{164}$ E. Appelt, ${ }^{165}$ A. G. Delannoy, ${ }^{165}$ S. Greene, ${ }^{165}$ A. Gurrola, ${ }^{165}$ R. Janjam, ${ }^{165}$ W. Johns,${ }^{165}$ C. Maguire, ${ }^{165}$ Y. Mao, ${ }^{165}$ A. Melo, ${ }^{165}$ H. Ni, ${ }^{165}$ P. Sheldon, ${ }^{165}$ B. Snook, ${ }^{165}$ S. Tuo, ${ }^{165}$ J. Velkovska, ${ }^{165}$ Q. Xu, ${ }^{165}$ M. W. Arenton, ${ }^{166}$ B. Cox ${ }^{166}$ B. Francis, ${ }^{166}$ J. Goodell, ${ }^{166}$ R. Hirosky, ${ }^{166}$ A. Ledovskoy ${ }^{166}$ H. Li,${ }^{166}$ C. Lin, ${ }^{166}$ C. Neu, ${ }^{166}$ T. Sinthuprasith, ${ }^{166}$ X. Sun, ${ }^{166}$ Y. Wang, ${ }^{166}$ E. Wolfe, ${ }^{166}$ J. Wood, ${ }^{166}$ F. Xia, ${ }^{166}$ C. Clarke, ${ }^{167}$ R. Harr, ${ }^{167}$ P. E. Karchin, ${ }^{167}$ 
C. Kottachchi Kankanamge Don, ${ }^{167}$ P. Lamichhane, ${ }^{167}$ J. Sturdy, ${ }^{167}$ D. A. Belknap, ${ }^{168}$ D. Carlsmith, ${ }^{168}$ M. Cepeda, ${ }^{168}$ S. Dasu, ${ }^{168}$ L. Dodd ${ }^{168}$ S. Duric, ${ }^{168}$ B. Gomber, ${ }^{168}$ M. Grothe, ${ }^{168}$ R. Hall-Wilton, ${ }^{168}$ M. Herndon, ${ }^{168}$ A. Hervé, ${ }^{168}$ P. Klabbers, ${ }^{168}$ A. Lanaro, ${ }^{168}$ A. Levine, ${ }^{168}$ K. Long, ${ }^{168}$ R. Loveless, ${ }^{168}$ A. Mohapatra, ${ }^{168}$ I. Ojalvo, ${ }^{168}$ T. Perry, ${ }^{168}$ G. A. Pierro, ${ }^{168}$ G. Polese, ${ }^{168}$ T. Ruggles, ${ }^{168}$ T. Sarangi, ${ }^{168}$ A. Savin, ${ }^{168}$ A. Sharma, ${ }^{168}$ N. Smith, ${ }^{168}$ W. H. Smith, ${ }^{168}$ D. Taylor, ${ }^{168}$ and N. Woods ${ }^{168}$

(CMS Collaboration)

\author{
${ }^{1}$ Yerevan Physics Institute, Yerevan, Armenia \\ ${ }^{2}$ Institut für Hochenergiephysik der OeAW, Wien, Austria \\ ${ }^{3}$ National Centre for Particle and High Energy Physics, Minsk, Belarus \\ ${ }^{4}$ Universiteit Antwerpen, Antwerpen, Belgium \\ ${ }^{5}$ Vrije Universiteit Brussel, Brussel, Belgium \\ ${ }^{6}$ Université Libre de Bruxelles, Bruxelles, Belgium \\ ${ }^{7}$ Ghent University, Ghent, Belgium \\ ${ }^{8}$ Université Catholique de Louvain, Louvain-la-Neuve, Belgium \\ ${ }^{9}$ Université de Mons, Mons, Belgium \\ ${ }^{10}$ Centro Brasileiro de Pesquisas Fisicas, Rio de Janeiro, Brazil \\ ${ }^{11}$ Universidade do Estado do Rio de Janeiro, Rio de Janeiro, Brazil \\ ${ }^{12 a}$ Universidade Estadual Paulista, São Paulo, Brazil \\ ${ }^{12 \mathrm{~b}}$ Universidade Federal do ABC, São Paulo, Brazil \\ ${ }^{13}$ Institute for Nuclear Research and Nuclear Energy, Sofia, Bulgaria \\ ${ }^{14}$ University of Sofia, Sofia, Bulgaria \\ ${ }^{15}$ Institute of High Energy Physics, Beijing, China \\ ${ }^{16}$ State Key Laboratory of Nuclear Physics and Technology, Peking University, Beijing, China \\ ${ }^{17}$ Universidad de Los Andes, Bogota, Colombia \\ ${ }^{18}$ University of Split, Faculty of Electrical Engineering, Mechanical Engineering and Naval Architecture, \\ Split, Croatia \\ ${ }^{19}$ University of Split, Faculty of Science, Split, Croatia \\ ${ }^{20}$ Institute Rudjer Boskovic, Zagreb, Croatia \\ ${ }^{21}$ University of Cyprus, Nicosia, Cyprus \\ ${ }^{22}$ Charles University, Prague, Czech Republic \\ ${ }^{23}$ Academy of Scientific Research and Technology of the Arab Republic of Egypt, Egyptian Network of \\ High Energy Physics, Cairo, Egypt \\ ${ }^{24}$ National Institute of Chemical Physics and Biophysics, Tallinn, Estonia \\ ${ }^{25}$ Department of Physics, University of Helsinki, Helsinki, Finland \\ ${ }^{26}$ Helsinki Institute of Physics, Helsinki, Finland \\ ${ }^{27}$ Lappeenranta University of Technology, Lappeenranta, Finland \\ ${ }^{28} \mathrm{DSM} / \mathrm{IRFU}, \mathrm{CEA} /$ Saclay, Gif-sur-Yvette, France \\ ${ }^{29}$ Laboratoire Leprince-Ringuet, Ecole Polytechnique, IN2P3-CNRS, Palaiseau, France \\ ${ }^{30}$ Institut Pluridisciplinaire Hubert Curien, Université de Strasbourg, Université de Haute Alsace \\ Mulhouse, CNRS/IN2P3, Strasbourg, France \\ ${ }^{31}$ Centre de Calcul de l'Institut National de Physique Nucleaire et de Physique des Particules, \\ CNRS/IN2P3, Villeurbanne, France \\ ${ }^{32}$ Université de Lyon, Université Claude Bernard Lyon 1, CNRS-IN2P3, \\ Institut de Physique Nucléaire de Lyon, Villeurbanne, France \\ ${ }^{33}$ Georgian Technical University, Tbilisi, Georgia \\ ${ }^{34}$ Tbilisi State University, Tbilisi, Georgia \\ ${ }^{35}$ RWTH Aachen University, I. Physikalisches Institut, Aachen, Germany \\ ${ }^{36}$ RWTH Aachen University, III. Physikalisches Institut A, Aachen, Germany \\ ${ }^{37}$ RWTH Aachen University, III. Physikalisches Institut B, Aachen, Germany \\ ${ }^{38}$ Deutsches Elektronen-Synchrotron, Hamburg, Germany \\ ${ }^{39}$ University of Hamburg, Hamburg, Germany \\ ${ }^{40}$ Institut für Experimentelle Kernphysik, Karlsruhe, Germany \\ ${ }^{41}$ Institute of Nuclear and Particle Physics (INPP), NCSR Demokritos, Aghia Paraskevi, Greece \\ ${ }^{42}$ University of Athens, Athens, Greece \\ ${ }^{43}$ University of Ioánnina, Ioánnina, Greece \\ ${ }^{44}$ Wigner Research Centre for Physics, Budapest, Hungary \\ ${ }^{45}$ Institute of Nuclear Research ATOMKI, Debrecen, Hungary
}


${ }^{46}$ University of Debrecen, Debrecen, Hungary

${ }^{47}$ National Institute of Science Education and Research, Bhubaneswar, India

${ }^{48}$ Panjab University, Chandigarh, India

${ }^{49}$ University of Delhi, Delhi, India

${ }^{50}$ Saha Institute of Nuclear Physics, Kolkata, India

${ }^{51}$ Bhabha Atomic Research Centre, Mumbai, India

${ }^{52}$ Tata Institute of Fundamental Research, Mumbai, India

${ }^{53}$ Indian Institute of Science Education and Research (IISER), Pune, India

${ }^{54}$ Institute for Research in Fundamental Sciences (IPM), Tehran, Iran

${ }^{55}$ University College Dublin, Dublin, Ireland

${ }^{56 a}$ INFN Sezione di Bari, Bari, Italy

${ }^{56 \mathrm{~b}}$ Università di Bari, Bari, Italy

${ }^{56 c}$ Politecnico di Bari, Bari, Italy

${ }^{57 \mathrm{a}}$ INFN Sezione di Bologna, Bologna, Italy

${ }^{57 \mathrm{~b}}$ Università di Bologna, Bologna, Italy

${ }^{58 a}$ INFN Sezione di Catania, Catania, Italy

${ }^{58 \mathrm{~b}}$ Università di Catania, Catania, Italy

${ }^{59 a}$ INFN Sezione di Firenze, Firenze, Italy

${ }^{59 \mathrm{~b}}$ Università di Firenze, Firenze, Italy

${ }^{60}$ INFN Laboratori Nazionali di Frascati, Frascati, Italy

${ }^{61 \mathrm{a} I N F N}$ Sezione di Genova, Genova, Italy

${ }^{61 \mathrm{~b}}$ Università di Genova, Genova, Italy

${ }^{62 a}$ INFN Sezione di Milano-Bicocca, Milano, Italy

${ }^{62 \mathrm{~b}}$ Università di Milano-Bicocca, Milano, Italy

${ }^{63 a}$ INFN Sezione di Napoli, Napoli, Italy

${ }^{63 \mathrm{~b}}$ Università di Napoli 'Federico II', Napoli, Italy

${ }^{63 \mathrm{c}}$ Università della Basilicata, Potenza, Italy

${ }^{63 \mathrm{~d}}$ Università G. Marconi, Roma, Italy

${ }^{64 a}$ INFN Sezione di Padova, Padova, Italy

${ }^{64 \mathrm{~b}}$ Università di Padova, Padova, Italy

${ }^{64 \mathrm{c}}$ Università di Trento, Trento, Italy

${ }^{65}$ INFN Sezione di Pavia, Pavia, Italy

${ }^{65 \mathrm{~b}}$ Università di Pavia, Pavia, Italy

${ }^{66 \mathrm{a}}$ INFN Sezione di Perugia, Perugia, Italy

${ }^{66 \mathrm{~b}}$ Università di Perugia, Perugia, Italy

${ }^{67 a}$ INFN Sezione di Pisa, Pisa, Italy

${ }^{67 \mathrm{~b}}$ Università di Pisa, Pisa, Italy

${ }^{67 \mathrm{c} S c u o l a}$ Normale Superiore di Pisa, Pisa, Italy

${ }^{68 \mathrm{a}}$ INFN Sezione di Roma

${ }^{68 \mathrm{~b}}$ Università di Roma

${ }^{69 \mathrm{a}}$ INFN Sezione di Torino, Torino, Italy

${ }^{69 \mathrm{~b}}$ Università di Torino, Torino, Italy

${ }^{69 \mathrm{c}}$ Università del Piemonte Orientale, Novara, Italy

${ }^{70 a}$ INFN Sezione di Trieste, Trieste, Italy

${ }^{70 \mathrm{~b}}$ Università di Trieste, Trieste, Italy

${ }^{71}$ Kangwon National University, Chunchon, Korea

${ }^{72}$ Kyungpook National University, Daegu, Korea

${ }^{73}$ Chonbuk National University, Jeonju, Korea

${ }^{74}$ Chonnam National University, Institute for Universe and Elementary Particles, Kwangju, Korea

${ }^{75}$ Korea University, Seoul, Korea

${ }^{76}$ Seoul National University, Seoul, Korea

${ }^{77}$ University of Seoul, Seoul, Korea

${ }^{78}$ Sungkyunkwan University, Suwon, Korea

${ }^{79}$ Vilnius University, Vilnius, Lithuania

${ }^{80}$ National Centre for Particle Physics, Universiti Malaya, Kuala Lumpur, Malaysia

${ }^{81}$ Centro de Investigacion y de Estudios Avanzados del IPN, Mexico City, Mexico

${ }^{82}$ Universidad Iberoamericana, Mexico City, Mexico

${ }^{83}$ Benemerita Universidad Autonoma de Puebla, Puebla, Mexico

${ }^{84}$ Universidad Autónoma de San Luis Potosí, San Luis Potosí, Mexico

${ }^{85}$ University of Auckland, Auckland, New Zealand 
${ }^{86}$ University of Canterbury, Christchurch, New Zealand

${ }^{87}$ National Centre for Physics, Quaid-I-Azam University, Islamabad, Pakistan

${ }^{88}$ National Centre for Nuclear Research, Swierk, Poland

${ }^{89}$ Institute of Experimental Physics, Faculty of Physics, University of Warsaw, Warsaw, Poland

${ }^{90}$ Laboratório de Instrumentação e Física Experimental de Partículas, Lisboa, Portugal

${ }^{91}$ Joint Institute for Nuclear Research, Dubna, Russia

${ }^{92}$ Petersburg Nuclear Physics Institute, Gatchina (St. Petersburg), Russia

${ }^{93}$ Institute for Nuclear Research, Moscow, Russia

${ }^{94}$ Institute for Theoretical and Experimental Physics, Moscow, Russia

${ }^{95}$ National Research Nuclear University 'Moscow Engineering Physics Institute' (MEPhI), Moscow, Russia

${ }^{96}$ P.N. Lebedev Physical Institute, Moscow, Russia

${ }^{97}$ Skobeltsyn Institute of Nuclear Physics, Lomonosov Moscow State University, Moscow, Russia

${ }^{98}$ State Research Center of Russian Federation, Institute for High Energy Physics, Protvino, Russia

${ }^{99}$ University of Belgrade, Faculty of Physics and Vinca Institute of Nuclear Sciences, Belgrade, Serbia

${ }^{100}$ Centro de Investigaciones Energéticas Medioambientales y Tecnológicas (CIEMAT), Madrid, Spain

${ }^{101}$ Universidad Autónoma de Madrid, Madrid, Spain

${ }^{102}$ Universidad de Oviedo, Oviedo, Spain

${ }^{103}$ Instituto de Física de Cantabria (IFCA), CSIC-Universidad de Cantabria, Santander, Spain

${ }^{104}$ CERN, European Organization for Nuclear Research, Geneva, Switzerland

${ }^{105}$ Paul Scherrer Institut, Villigen, Switzerland

${ }^{106}$ Institute for Particle Physics, ETH Zurich, Zurich, Switzerland

${ }^{107}$ Universität Zürich, Zurich, Switzerland

${ }^{108}$ National Central University, Chung-Li, Taiwan

${ }^{109}$ National Taiwan University (NTU), Taipei, Taiwan

${ }^{110}$ Chulalongkorn University, Faculty of Science, Department of Physics, Bangkok, Thailand

${ }^{111}$ Cukurova University, Adana, Turkey

${ }^{112}$ Middle East Technical University, Physics Department, Ankara, Turkey

${ }^{113}$ Bogazici University, Istanbul, Turkey

${ }^{114}$ Istanbul Technical University, Istanbul, Turkey

${ }^{115}$ Institute for Scintillation Materials of National Academy of Science of Ukraine, Kharkov, Ukraine

${ }^{116}$ National Scientific Center, Kharkov Institute of Physics and Technology, Kharkov, Ukraine

${ }^{117}$ University of Bristol, Bristol, United Kingdom

${ }^{118}$ Rutherford Appleton Laboratory, Didcot, United Kingdom

${ }^{119}$ Imperial College, London, United Kingdom

${ }^{120}$ Brunel University, Uxbridge, United Kingdom

${ }^{121}$ Baylor University, Waco, USA

${ }^{122}$ The University of Alabama, Tuscaloosa, USA

${ }^{123}$ Boston University, Boston, USA

${ }^{124}$ Brown University, Providence, USA

${ }^{125}$ University of California, Davis, Davis, USA

${ }^{126}$ University of California, Los Angeles, USA

${ }^{127}$ University of California, Riverside, Riverside, USA

${ }^{128}$ University of California, San Diego, La Jolla, USA

${ }^{129}$ University of California, Santa Barbara, Santa Barbara, USA

${ }^{130}$ California Institute of Technology, Pasadena, USA

${ }^{131}$ Carnegie Mellon University, Pittsburgh, USA

${ }^{132}$ University of Colorado Boulder, Boulder, USA

${ }^{133}$ Cornell University, Ithaca, USA

${ }^{134}$ Fermi National Accelerator Laboratory, Batavia, USA

${ }^{135}$ University of Florida, Gainesville, USA

${ }^{136}$ Florida International University, Miami, USA

${ }^{137}$ Florida State University, Tallahassee, USA

${ }^{138}$ Florida Institute of Technology, Melbourne, USA

${ }^{139}$ University of Illinois at Chicago (UIC), Chicago, USA

${ }^{140}$ The University of Iowa, Iowa City, USA

${ }^{141}$ Johns Hopkins University, Baltimore, USA

${ }^{142}$ The University of Kansas, Lawrence, USA

${ }^{143}$ Kansas State University, Manhattan, USA

${ }^{144}$ Lawrence Livermore National Laboratory, Livermore, USA 


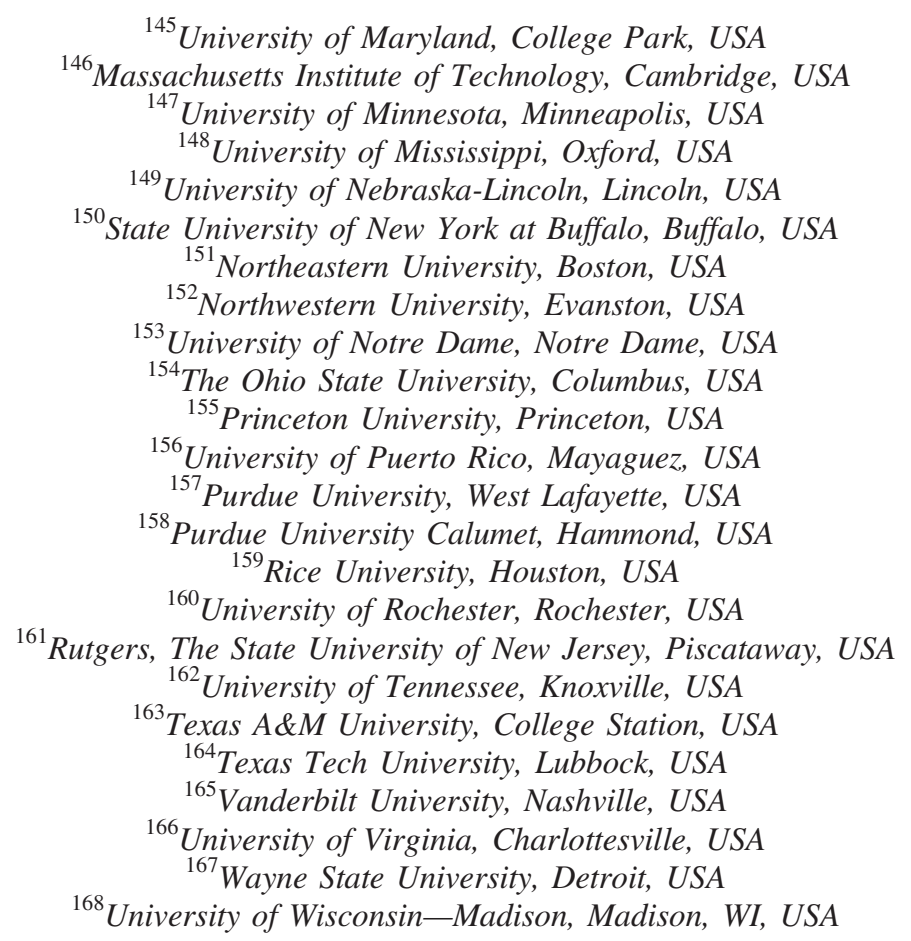

${ }^{\mathrm{a}}$ Deceased.

${ }^{\mathrm{b}}$ Also at Vienna University of Technology, Vienna, Austria.

${ }^{c}$ Also at CERN, European Organization for Nuclear Research, Geneva, Switzerland.

${ }^{\mathrm{d}}$ Also at State Key Laboratory of Nuclear Physics and Technology, Peking University, Beijing, China.

${ }^{\mathrm{e}}$ Also at Institut Pluridisciplinaire Hubert Curien, Université de Strasbourg, Université de Haute Alsace Mulhouse, CNRS/IN2P3, Strasbourg, France.

${ }^{\mathrm{f}}$ Also at National Institute of Chemical Physics and Biophysics, Tallinn, Estonia.

${ }^{g}$ Also at Skobeltsyn Institute of Nuclear Physics, Lomonosov Moscow State University, Moscow, Russia.

${ }^{\mathrm{h}}$ Also at Universidade Estadual de Campinas, Campinas, Brazil.

${ }^{\mathrm{i}}$ Also at Centre National de la Recherche Scientifique (CNRS)_IN2P3, Paris, France.

${ }^{\mathrm{j}}$ Also at Laboratoire Leprince-Ringuet, Ecole Polytechnique, IN2P3-CNRS, Palaiseau, France.

${ }^{\mathrm{k}}$ Also at Joint Institute for Nuclear Research, Dubna, Russia.

${ }^{1}$ Also at Suez University, Suez, Egypt.

${ }^{\mathrm{m}}$ Also at British University in Egypt, Cairo, Egypt.

${ }^{\mathrm{n}}$ Also at Cairo University, Cairo, Egypt.

${ }^{\circ}$ Also at Fayoum University, El-Fayoum, Egypt.

${ }^{\mathrm{p}}$ Also at Université de Haute Alsace, Mulhouse, France.

${ }^{\mathrm{q}}$ Also at Tbilisi State University, Tbilisi, Georgia.

${ }^{\mathrm{r}}$ Also at RWTH Aachen University, III. Physikalisches Institut A, Aachen, Germany.

${ }^{\mathrm{s}}$ Also at Indian Institute of Science Education and Research, Bhopal, India.

${ }^{\mathrm{t}}$ Also at University of Hamburg, Hamburg, Germany.

"Also at Brandenburg University of Technology, Cottbus, Germany.

${ }^{v}$ Also at Institute of Nuclear Research ATOMKI, Debrecen, Hungary.

${ }^{\mathrm{w}}$ Also at Eötvös Loránd University, Budapest, Hungary.

${ }^{\mathrm{x}}$ Also at University of Debrecen, Debrecen, Hungary.

${ }^{y}$ Also at Wigner Research Centre for Physics, Budapest, Hungary.

${ }^{\mathrm{z}}$ Also at University of Visva-Bharati, Santiniketan, India.

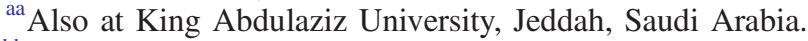

${ }^{\mathrm{bb}}$ Also at University of Ruhuna, Matara, Sri Lanka.

${ }^{c c}$ Also at Isfahan University of Technology, Isfahan, Iran.

${ }^{\mathrm{dd}}$ Also at University of Tehran, Department of Engineering Science, Tehran, Iran.

${ }^{e e}$ Also at Plasma Physics Research Center, Science and Research Branch, Islamic Azad University, Tehran, Iran.

${ }^{\mathrm{ff}}$ Also at Università degli Studi di Siena, Siena, Italy.

${ }^{g g}$ Also at Purdue University, West Lafayette, USA.

${ }^{\text {hh }}$ Also at International Islamic University of Malaysia, Kuala Lumpur, Malaysia. 
${ }^{\text {ii }}$ Also at Malaysian Nuclear Agency, MOSTI, Kajang, Malaysia.

${ }^{j j}$ Also at Consejo Nacional de Ciencia y Tecnología, Mexico city, Mexico.

${ }^{\mathrm{kk}}$ Also at Warsaw University of Technology, Institute of Electronic Systems, Warsaw, Poland.

${ }^{11}$ Also at Institute for Nuclear Research, Moscow, Russia.

${ }^{\mathrm{mm}}$ Also at National Research Nuclear University 'Moscow Engineering Physics Institute' (MEPhI), Moscow, Russia.

${ }^{\mathrm{nn}}$ Also at St. Petersburg State Polytechnical University, St. Petersburg, Russia.

${ }^{\circ o}$ Also at California Institute of Technology, Pasadena, USA.

${ }^{p p}$ Also at Faculty of Physics, University of Belgrade, Belgrade, Serbia.

${ }^{\mathrm{qq}}$ Also at INFN Sezione di Roma, Università di Roma, Roma, Italy.

${ }^{\mathrm{rr}}$ Also at National Technical University of Athens, Athens, Greece.

${ }^{\text {ss }}$ Also at Scuola Normale e Sezione dell'INFN, Pisa, Italy.

${ }^{t t}$ Also at University of Athens, Athens, Greece.

${ }^{\mathrm{uu}}$ Also at Institute for Theoretical and Experimental Physics, Moscow, Russia.

${ }^{\mathrm{v}}$ Also at Albert Einstein Center for Fundamental Physics, Bern, Switzerland.

${ }^{\text {ww }}$ Also at Adiyaman University, Adiyaman, Turkey.

${ }^{\mathrm{xx}}$ Also at Mersin University, Mersin, Turkey.

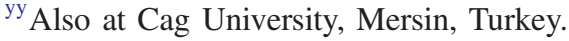

${ }^{\mathrm{zz}}$ Also at Piri Reis University, Istanbul, Turkey.

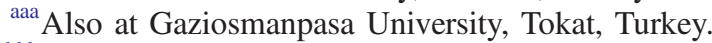

${ }^{b b b}$ Also at Ozyegin University, Istanbul, Turkey.

${ }^{c c c}$ Also at Izmir Institute of Technology, Izmir, Turkey.

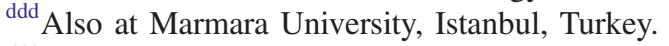

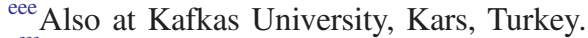

${ }^{\mathrm{fff}}$ Also at Mimar Sinan University, Istanbul, Istanbul, Turkey.

${ }^{\text {ggg }}$ Also at Yildiz Technical University, Istanbul, Turkey.

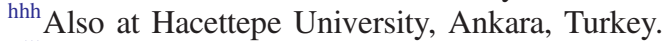

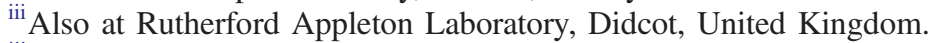

jij Also at School of Physics and Astronomy, University of Southampton, Southampton, United Kingdom.

${ }^{k k k}$ Also at Instituto de Astrofísica de Canarias, La Laguna, Spain.

${ }^{111}$ Also at Utah Valley University, Orem, USA.

${ }^{\mathrm{mmm}}$ Also at University of Belgrade, Faculty of Physics and Vinca Institute of Nuclear Sciences, Belgrade, Serbia.

${ }^{n n n}$ Also at Facoltà Ingegneria, Università di Roma, Roma, Italy.

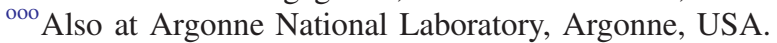

${ }^{p p p}$ Also at Erzincan University, Erzincan, Turkey.

${ }^{\mathrm{qqq}}$ Also at Texas A\&M University at Qatar, Doha, Qatar.

${ }^{\mathrm{rrr}}$ Also at Kyungpook National University, Daegu, Korea. 